Discussion

Papers

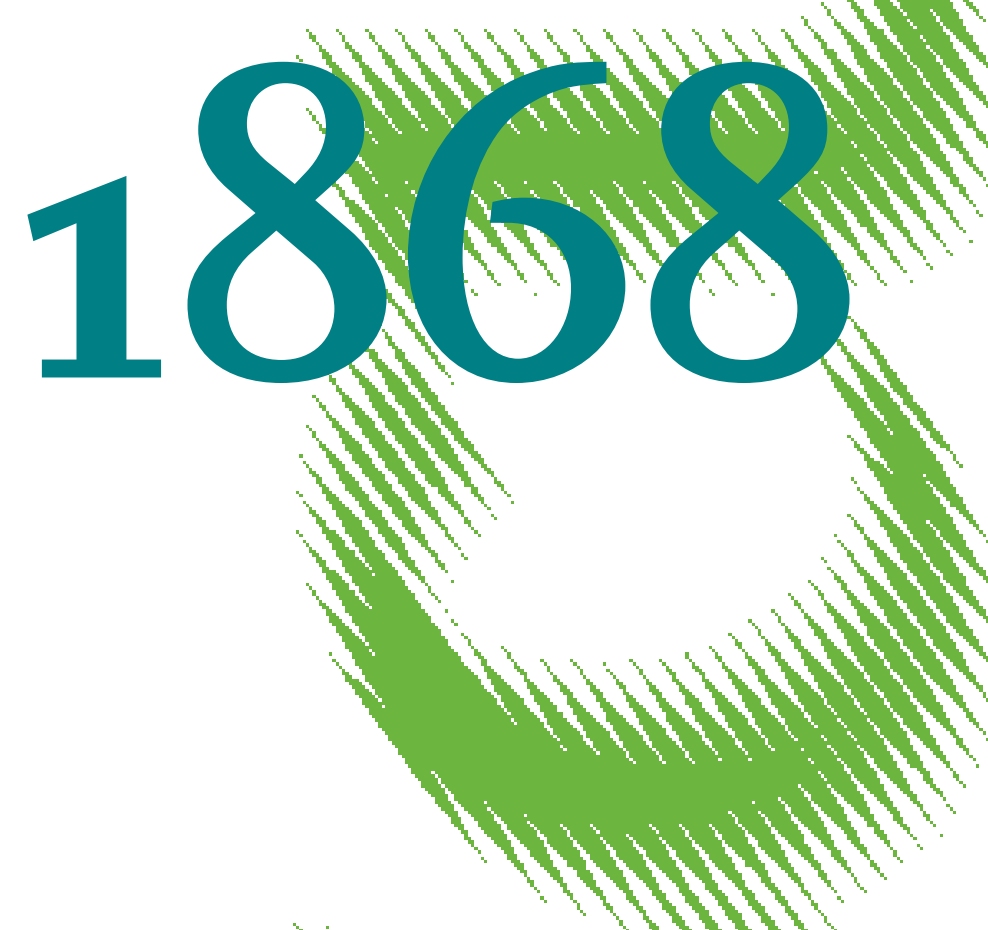

Knowledge-Based Capital and Productivity Divergence 
Opinions expressed in this paper are those of the author(s) and do not necessarily reflect views of the institute.

IMPRESSUM

(C) DIW Berlin, 2020

DIW Berlin

German Institute for Economic Research

Mohrenstr. 58

10117 Berlin

Tel. +49 (30) $89789-0$

Fax +49 (30) $89789-200$

http://www.diw.de

ISSN electronic edition 1619-4535

Papers can be downloaded free of charge from the DIW Berlin website:

http://www.diw.de/discussionpapers

Discussion Papers of DIW Berlin are indexed in RePEc and SSRN:

http://ideas.repec.org/s/diw/diwwpp.html

http://www.ssrn.com/link/DIW-Berlin-German-Inst-Econ-Res.html 


\title{
Knowledge-based capital and productivity divergence ${ }^{\sqrt{3}}$
}

\author{
Marie Le Mouel ${ }^{\mathrm{a}, *}$, Alexander Schiersch ${ }^{\mathrm{b}}$ \\ ${ }^{a}$ Tilburg University, Warandelaan 2, 5037 AB Tilburg, The Netherlands \\ ${ }^{b}$ DIW Berlin, Mohrenstr. 58, 10117 Berlin, Germany
}

\begin{abstract}
Understanding the causes of the slowdown in aggregate productivity growth is key to maintaining the competitiveness of advanced economies and ensuring long-term economic prosperity. This paper is the first to provide evidence that investment in Knowledge-Based Capital (KBC), despite having a positive effect on productivity at the micro level, is a driver of the weak productivity performance at the aggregate level, by accentuating divergence between a group of "frontier" firms and the rest of the economy. Using detailed firm-level administrative data for Germany, we find evidence that the effect of $\mathrm{KBC}$ on productivity is heterogeneous across firms within industries: this effect is 3 times larger for firms in the top quintile of the KBC distribution compared to firms in the bottom quintile of the KBC distribution. We document the existence of divergence in productivity growth between top KBC users and the rest of firms at the industry level, and find that industries where this gap is larger are also those industries where the heterogeneity in the effect of $\mathrm{KBC}$ is highest and where average productivity growth was lower. The evidence hence supports the view that the use of KBC plays a role in explaining weak productivity growth, by accentuating differences between firms.
\end{abstract}

Keywords: Knowledge-Based Capital, Firm dynamics, Productivity divergence

JEL codes: D24, L25, O14, O30, O47

\footnotetext{
${ }^{\sqrt{4}}$ We thank Tomaso Duso, Alexandra Spitz-Oener, Axel Werwatz, Michael Polder, Marianne Saam, Pio Baake, Hannes Ullrich, Lenka Fiala and participants at the DIW Berlin, Groningen University, Banque de France, Bochum University, and the IARIW $35^{\text {th }}$ General conference for helpful comments and suggestions. Special thanks are due to Ramona Vosshage and her team at the Federal Statistical Office Berlin-Brandenburg. Funding: This research was partly funded by the German Ministry of Economics and Energy through the project: "Wissensbasiertes Kapital in Deutschland: Analyse zu Produktivitäts- und Wachstumseffekten und Erstellung eines Indikatorsystems".

${ }^{*}$ Corresponding author

Email addresses: m.a.f.lemouel@tilburguniversity.edu (Marie Le Mouel), aschiersch@diw.de (Alexander Schiersch)
} 


\section{Introduction}

The well-documented slowdown in productivity growth in advanced economies has sparked a vivid debate around the question of whether the engine of economic growth has stalled. Across the OECD, the average yearly growth rate of labour productivity declined from 2.3\% for the period 1995-2004 to 1.1\% for the period 2005-2015 (Syverson, 2017). Yet, investments in productivity-enhancing factors, such as Research \& Development (R\&D) and patents, software, designs, brands and trademarks, and organisational practices, collectively constituting what we will refer to as Knowledge-Based Capital (KBC), have kept increasing at a steady rate. Corrado et al. (2016) report that the annual growth rate of KBC investment, over the period 2000-2013, was $2.6 \%$ in the United States and 2.0\% across 14 member countries of the European Union (EU14), outpacing growth in tangible investment by around 1.5 percentage points in both regions. Moreover, the level of investment in KBC has overtaken that in tangible capital in the United States, the United Kingdom and France, and is around $70 \%$ of the level for tangible capital in other industrialized countries.

Schumpeterian growth models can help shed light on this apparent paradox. In these models, the main mechanism driving aggregate growth is the ability of firms to reap a reward from their innovation effort. Markets need to be contestable and innovators need to be able to capture markets when they invent superior products and services. However, recent evidence reported by Garcia-Macia et al. (2019) suggests that the contribution of entrants and of creative destruction to growth has declined between the period 2003-2013 and the period 19831993, while the contribution of incumbents' own innovation has increased. The weakening of the contestability of markets can also be seen in a number of trends succinctly summarised by Akcigit and Ates (2019a,b). Market concentration, markups and profits have increased (Autor et al., 2020; De Loecker et al., 2020), while firm entry rates, the share of young firms in economic activity and job reallocation rates have declined (Decker et al., 2016b,c). Finally, productivity is diverging between a group of top-performers on the frontier and the bulk of firms (Andrews et al., 2019).

In addition to documenting growing divergence between firms along multiple dimensions, the studies cited above also discuss various mechanisms which might underpin these developments. Inter alia, Autor et al. (2020) point to the competitive advantage of highly productive firms that seize larger shares of the market, while De Loecker et al. (2020) attribute the increase in markups, especially pronounced in larger firms, to the decreasing intensity of competition. Andrews et al. (2019) test the relevance of labour and product market regulations and of a fall in competitive pressure as explanations for the increase in divergence, as these can lock resources in less productive firms and hinder their reallocation towards new and more productive companies.

The aforementioned studies also discuss, but do not directly test, that the increased importance of KBC plays a role in this development. The present research contributes to this discussion on the productivity slowdown and growing productivity divergence, by explicitly exploring the role of KBC. We postulate that the effect of $\mathrm{KBC}$ is heterogeneous across firms, in particular that firms with large $\mathrm{KBC}$ stocks benefit more from further 
investment than firms with smaller stocks. We furthermore analyse the relationship between the use of KBC at the firm level and the trends in productivity divergence between frontier firms and the rest. Finally, we link the observed productivity dispersion associated with KBC to average productivity growth.

We bring together firm-level productivity estimation in the vein of Doraszelski and Jaumandreu (2013) and Ackerberg et al. (2015) and the methodology of Andrews et al. (2019), who provide compelling evidence of divergence between firms on the global technology frontier and the rest of firms. The analysis uses firm-level administrative data for Germany for the period 2003-2014, covering 47 detailed industries in the manufacturing sector, and knowledge-intensive and other services. The data contain firm expenditures on four KBC assets: software, R\&D, organizational capital and Intellectual Property Products (IPP), such as patents, licenses or trademarks. These are capitalized and aggregated into a total stock of $\mathrm{KBC}$ for each firm.

The first stage of the analysis consists of estimating the role of $\mathrm{KBC}$ on firm-level productivity, using the control function approach of Doraszelski and Jaumandreu (2013) and Ackerberg et al. (2015). We model the role of the KBC stock as directly affecting the evolution of productivity, rather than as an input of production, and thereby control for the endogeneity of KBC. Furthermore, a flexible functional form is adopted to govern the relationship between firm-level productivity and KBC. This allows us to recover firm-specific elasticities of $\mathrm{KBC}$ on productivity, and to explore the heterogeneity in this effect. The estimations are carried out separately for each detailed industry, allowing for different production structures.

The results show that the effect of KBC on firm-level productivity is positive across all industries. Moreover, they confirm the presence of increasing returns to the size of a firm's stock of KBC in all but 6 of the 47 industries. Stark differences in firm-specific elasticities of KBC can be found between firms in the top and in the bottom quintiles of the $\mathrm{KBC}$ distribution. Across both manufacturing and services, the elasticity of $\mathrm{KBC}$ is around 3 times larger for firms with the largest KBC stocks compared to firms with the smallest KBC stocks. Finally, the elasticity of $\mathrm{KBC}$ is negative in the bottom quintile of the distribution of $\mathrm{KBC}$ stock in 14 industries. These results confirm the important heterogeneity in the ability of firms to reap the benefits of their KBC investments, further highlighting the productivity disadvantage of laggard firms who fail to reach a minimum scale of KBC. ${ }^{1}$

The second stage of the analysis relates these firm-level findings to aggregate productivity trends. The focus is on the divergence of productivity between frontier firms and the rest, as the key mechanism being explored is the ability of frontier firms to act as a locomotive for the rest of the economy. The present study adds to the current literature, which has analysed the evolution of the productivity distribution, by shedding light on the development of productivity along a different distribution, that of KBC stock. Adopting the framework of Andrews et al. (2019), two sets of frontier firms are defined: those in the top 5\% of, on the one hand, the productivity distribution, and on the other, the distribution of KBC stock, for each industry and year. Contrary

\footnotetext{
${ }^{1}$ Factors hampering the ability of firms to scale up their KBC use include financing constraints, lack of access to skilled labour, and incentives to invest in absorbing knowledge spillovers, rather than in building up own capacity (Haskel and Westlake, 2018).
} 
to the results of Andrews et al. (2019) using international data, the German economy does not display a strong pattern of productivity divergence between the top productive firms and the rest. However, the productivity of firms on the KBC-frontier did grow faster than that of the other firms, in both manufacturing and services. ${ }^{2}$

Correlating the size of the productivity gap between frontier firms and the rest with the elasticities of KBC obtained from the estimations at the detailed industry level further highlights the important role of KBC. Industries where the gap in productivity growth was largest are those industries where the average effect of KBC on productivity was larger, and are also those industries where the difference in the elasticity of KBC between frontier firms and the rest was most pronounced. Finally, this study sheds light on whether this KBC-driven divergence is related to the productivity slowdown, by replicating the analysis of Andrews et al. (2019). We regress the 3-year productivity growth for each industry on the increase in the productivity gap, and find that industries where the productivity gap was more marked are those industries that had lower average productivity growth. This result suggests that the heterogeneous effect of $\mathrm{KBC}$ on productivity at the firm level can have detrimental effects on productivity growth at the aggregate level.

The remainder of the paper is structured as follows. In Section 2, we discuss in more detail the theoretical literature setting out the mechanisms linking KBC investments to productivity divergence and lower growth, and highlight our contributions to the literature. Section 3 describes the dataset and the measurement of $\mathrm{KBC}$ variables. Section 4 presents our structural model of production and the associated estimation strategy used to recover firm-level productivity and KBC elasticity. In Section 5, we discuss the results of the structural estimation, focusing on the effect of KBC on firm-level productivity, and some robustness estimations. In Section 6 , we report the industry dynamics of productivity growth, on and off the frontier, and analyse its relationship to KBC. Finally, Section 7 presents our concluding remarks.

\section{Related Literature}

The present analysis is motivated by recent developments in the Schumpeterian growth literature, which formalize the mechanisms by which an increasing use of KBC in the economy results in "best versus rest" dynamics and lowers overall productivity performance. These dynamics arise from the special nature of knowledge-based assets that distinguish them from physical capital (Haskel and Westlake, 2018).

KBC needs to be construed as a bundle of assets, that encompass three broad categories (Corrado et al., 2005, 2009): computerized information, which covers all information susceptible to be digitalised; innovative property, which is the knowledge, scientific or otherwise, to develop new products; and economic competencies, which is the knowledge embedded in the employees and organisational structures of firms. These different types

\footnotetext{
${ }^{2}$ The lack of divergence along the productivity frontier mitigates the concern, pronounced for the American economy, that the over performance of top productive firms might be due to factors such as market power, weak antitrust enforcement, or regulatory capture (Covarrubias et al., 2019).
} 
of expenditures exhibit strong complementarities. For example, Bloom et al. (2013) and Schivardi and Schmitz (2019) show, for the United States, and for Germany, Italy, Spain and Portugal respectively, that Information and Communication Technology (ICT) investments need to be accompanied by organizational changes for firms to witness productivity gains. More generally, Brynjolfsson et al. (2020) argue that the roll-out of a new General Purpose Technology (GPT), such as ICT or Artificial Intelligence, requires complementary investments in intangible capital, especially organizational practices and human capital, which delay realized productivity gains.

Although not explicitly formulated in terms of KBC investments, the models of Akcigit and Ates (2019a) and Benhabib et al. (2019) demonstrate that a reduction in the ability or incentives of followers to catch-up with the frontier will accentuate the gap with leaders and diminish the hopes of followers to leapfrog to the frontier level of technology and seize the market. Akcigit and Ates (2019a) assume an exogenous slowdown in knowledge diffusion, while Benhabib et al. (2019) assume laggards can pay a cost to adopt a new technology from the distribution of existing technologies. The necessity of making investments in complementary assets can be interpreted as an increase in the costs of technology adoption in these models, and will stretch the aggregate productivity distribution and reduce the incentives of frontier firms to innovate.

Emphasizing another mechanism, the models of Aghion et al. (2019) and DeRidder (2019) rely on the nonrival nature of the knowledge embedded in $\mathrm{KBC}$, which creates strong economies of scale. Investing in $\mathrm{KBC}$ represents a fixed cost that allows firms to reduce the marginal costs of production. ${ }^{3}$ In addition to innovation efforts that improve product quality, these models add a second mechanism. Firms differ in their process efficiency, allowing more efficient firms to produce at lower marginal costs and therefore to offer lower prices for the same level of quality as rivals. The key difference between the two approaches is their assumption on the source of efficiency differences between firms. Aghion et al. (2019) assume firms differ according to a time-invariant efficiency level, whereas DeRidder (2019) endogenises this parameter, by making it the result of firm spending on intangibles. This dichotomy between quality and efficiency is precisely what allows successful (i.e. high efficiency) incumbents to overcome competition from better quality innovators and stay in the market by offering equivalent or lower quality, at lower prices. This in turn decreases the incentives of both leaders and followers to invest in innovation, thereby lowering overall growth. ${ }^{4}$ Without going into the dynamics of creative destruction, Hsieh and Rossi-Hansberg (2019) propose a model where investments in assets that reduce marginal costs help dominant firms expand into new geographical markets and control a larger share of sales in

\footnotetext{
${ }^{3}$ This characteristic on its own strongly favours large incumbents, but it is further accentuated by the sunk nature of many of these investments, which result in firm-specific assets that are difficult to value outside the firm and thus to secure financing (Haskel and Westlake, 2018).

${ }^{4}$ Both of these models predict an initial surge in productivity, as the more efficient firms capture higher shares of markets or product lines, but once their dominant position is established, innovation effort decreases. The authors argue that this is consistent with the pattern of productivity growth in the United States, which saw a surge in productivity growth in the 1990s, as firms were heavily investing in ICT.
} 
their respective industries. The model allows the authors to explain the observed restructuring of many service industries in the United States, in particular retail and wholesale trade, leading them to argue that KBC has led to a new industrial revolution in many non-traded service sectors.

This pattern of increased productivity dispersion, and especially stronger performance in the upper tail of the distribution as compared to the average or median firm, has been consistently documented for a number of countries. Using the international Orbis database, Andrews et al. (2019, 2015), and Bahar (2018) are able to observe the performance of firms on the global frontier. In contrast, national studies, such as Faggio et al. (2010) for the United Kingdom, Decker et al. (2017) the United States, Cette et al. (2018) for France, and Polder et al. (2018) for the Netherlands, as well as Berlingieri et al. (2017) covering 16 countries, compare the performance of national leaders to the rest of firms. A commonly discussed explanation for this increasing divergence is the use of scalable assets, such as ICT and complementary intangible capital. Andrews et al. (2019) report that divergence is more pronounced in ICT-intensive industries, and Bahar (2018) that it is more pronounced in knowledge-intensive industries. ${ }^{5}$ At present, data limitations have not permitted an explicit test of the role of $\mathrm{KBC}$ in this phenomenon.

A growing number of studies have confirmed the overall positive influence of $\mathrm{KBC}$ on firm productivity and have decomposed the individual contributions of different assets. ${ }^{6}$ See Crouzet and Eberly (2018) for the United States, Bontempi and Mairesse (2015) for France, Kaus et al. (2020) for Germany, Chappell and Jaffe (2018) for New Zealand, Siedschlag et al. (2017) for Ireland. Moreover, Crass and Peters (2014) and Mohnen et al. (2018) find evidence of complementarities between the different asset categories, and Marrocu et al. (2011) document the importance of spillovers arising from KBC spending by other firms in the same region, for a sample of six European countries. However, this literature has not explicitly explored the potentially heterogeneous effect of KBC across firms or its implications for productivity divergence.

The theoretical models outlined above and the empirically established findings on the positive relationship between $\mathrm{KBC}$ and productivity lead us to formulate and test the following two hypotheses:

Hypothesis 1. The effect of $K B C$ on productivity, though positive on average, is heterogeneous across firms and increases in the amount of $K B C$.

Hypothesis 2. The use of KBC in production exacerbates productivity differences between firms, weakening aggregate productivity growth, in both manufacturing and services.

We make a number of contributions to the literature presented above. Firstly, we provide evidence for the

${ }^{5}$ Bahar (2018) defines knowledge-intensive industries using the skill composition of the workforce from the US Bureau of Labor Statistics.

${ }^{6}$ The effect of individual elements of the broad bundle of $\mathrm{KBC}$ on firm performance has also been widely examined in isolation. For a review of the relationship between productivity and R\&D see Ugur et al. (2016), for ICT see Cardona et al. (2013), and for organisational capital see Bloom et al. (2017). 
key assumptions in the aforementioned theoretical models, notably that of DeRidder (2019). Specifically, we test for the presence of increasing returns to scale, and whether firms differ in their ability to reap the benefits of their KBC investments. Secondly, we exploit this heterogeneity in the returns to KBC by relating it to cross-industry differences in the degree of productivity divergence, adding new insights to the discussion on productivity divergence between frontier firms and followers, in particular on one of its potential causes. To the best of our knowledge, this is the first paper to relate firm-level investment in KBC to productivity divergence at the industry level.

Finally, we depart from the existing literature on the role of $\mathrm{KBC}$ on firm-level productivity in two important aspects. First, our dataset is representative within each 2-digit NACE industry, which allows us to account for different sectoral production functions, and covers four aggregate service sectors in addition to manufacturing. This is particularly important, as Corrado et al. (2016) report that the service sector amounts to $64 \%$ of market sector intangible investment in the US, and to $61 \%$ in the EU14. In this aspect, we differ from many of the studies reported above, which use the Community Innovation Survey. This latter source of data also provides information on the KBC activities of firms, but the yearly sample size of around 5,000 to 7,000 observations is not well suited for analyses at the two-digit industry level. Second, we differ from the literature methodologically, by modelling the contribution of $\mathrm{KBC}$ in the law of motion of productivity, rather than as an input of production, similarly to the approach of Doraszelski and Jaumandreu (2013) for R\&D. This assumption helps to control for the endogeneity of KBC by controlling for its contemporaneous effect on output, and imposing a one-year lag between changes in KBC stock and the resulting effect on productivity.

\section{Data}

The present analysis uses the firm-level datasets collected by the German Statistical Office ${ }^{7}$ and used as a source for the construction of the official System of National Accounts (SNA) aggregated data. To ensure the largest possible coverage of the German economy, we combine the AFiD Panel of Manufacturing Firms with the AFiD Panel of Service Firms. Both datasets consist of multiple sub-datasets, merged through unique firm identifiers. They are described in further detail in Appendix Sections A.1 and A.2.

The full dataset covers 50 detailed industries in Manufacturing, Transport and Warehousing, Information and Communication services, Business services, and Administrative activities. ${ }^{8}$ However, the following industries are dropped from the econometric analysis due to insufficient observations: the mining industry (B05 to B09), the

\footnotetext{
$\overline{{ }^{7} \text { Germany's system of statistical offices comprises } 16}$ state-level Statistical Offices, and an overarching German Federal Statistical Office. The Federal Office sets common guidelines and the state-level offices are responsible for data collection and processing, and retain ownership of the data. For simplicity we use Statistical Office without distinguishing between Federal and State level. See https://www.forschungsdatenzentrum.de/en for details.

${ }^{8}$ The industry information is presented under the classification WZ 2008, the German equivalent of ISIC Rev. 4, which came into force in 2008. For the period 2003-2007, the industry information is provided under the classification WZ 2003, equivalent to ISIC Rev. 3.1, and was converted to the WZ 2008 using a conversion table provided by Dierks et al. (2020).
} 
manufacturing of tobacco products (C12), and the manufacturing of refined petroleum products (C19). After data cleaning, the dataset contains a total of 1,019,811 firm-year observations. Complete coverage is available for the 2009-2014 period in the manufacturing sector, and for the 2003-2013 period for the services sectors. The panels are unbalanced.

We observe firm-level records of standard production variables such as gross value-added, number of employees, total wagebill, material and energy expenses, and investments in physical capital. ${ }^{9}$ Our data encompass the following KBC asset categories: software, IPP, R\&D, and organisational capital. The last of these variables is constructed from external information on firms' occupational structure obtained from the linked employeremployee dataset (LIAB) of the Institute for Employment Research (IAB).

\subsection{Investment in Knowledge-Based Capital}

The main source of information relating to investment in KBC are the AFiD Panels. Firms are asked to report their "Investments in intangible assets", which captures all expenditures on intangible assets as defined in the SNA. It is split between "Investments in concessions, patents, licenses, trademarks, etc." and "Investments in software". The first category measures investments in the IPP that firms can report on their balance sheets following the German accounting legislation. Investments in software only refer to external purchases of software and databases. ${ }^{10}$ This information on software and IPP investments is only collected from 2009 in the manufacturing sector, whereas it is available from 2003 in the services sector.

To capture firms' investments in economic competencies, we follow the widely-used occupation-based method of Corrado et al. (2009, 2005) (as in Niebel et al., 2017; Corrado et al., 2016; Miyagawa and Hisa, 2013; OECD, 2013; Le Mouel and Squicciarini, 2015). This approach relies on the assumption that managers devote $20 \%$ of their time to activities that improve the organisational structure of the company over the long run. Hence, $20 \%$ of managerial compensation should be considered long-lasting investments and be capitalised. We obtain the share of managerial wages in firms' total wage-bill from the LIAB database, and add this information to the AFiD Panel. Firm-specific investment in organisational capital is obtained as $20 \%$ of this estimated managerial compensation. The details of the methodology are provided in Appendix Section A.3. ${ }^{11}$

Finally, investment in innovative property is measured by expenditure on R\&D, obtained from the cost structure survey element of the AFiD Panel of Manufacturing Firms. All expenses incurred in the R\&D process, including investments in capital and intermediary inputs, are covered. This information is not available for firms

\footnotetext{
${ }^{9}$ The latter is measured as purchases, sales, new rentals and own-production of machines, tools, and buildings.

${ }^{10}$ Information on software developed in-house is only available from 2012 and is therefore not included in the analysis.

${ }^{11}$ Our estimates of firm-level organisational capital represent a lower bound compared to other methodologies present in the literature. For example, Corrado et al. (2009) also include purchases of management consulting services in their measure of investment in organisational capital. Alternatively, other authors, (e.g. Eisfeldt and Papanikolaou, 2013; Lev et al., 2009; Chen and Inklaar, 2015) use Sales, General and Administrative (SG\&A) expenses. However, our dataset does not contain information on either type of expenditures.
} 
in the services sectors and we estimate the labour costs of $R \& D$ activities in these sectors from the occupational information of the LIAB data in a similar fashion to investment in organisational capital, given that personnel expenditures represent around $60 \%$ of total R\&D costs in Germany (see OECD Research and Development statistics). We note that the interpretation of our results will therefore differ between the manufacturing and services sectors, as the total KBC stock is calculated with these different measures of R\&D. However, by estimating the model at the 2-digit industry level, we ensure that these different measures of R\&D are not pooled into the same estimation.

\subsection{Descriptive statistics}

Table 1 reports the mean and standard deviation of the production variables and selected percentiles of the distribution of KBC stock, investment and intensity (defined as the ratio of the stock of KBC over the stock of physical capital), by 1-digit industry. ${ }^{12}$ Our analysis uses the sum of the four assets as the main variable of interest, to acknowledge the fact that the optimal bundle of assets might differ across industries and firms. Our data replicate the commonly reported fact that the average firm in the manufacturing industry is larger in terms of value-added, labour, and physical capital than the average firm in the service industry.

The $10^{\text {th }}$ and $90^{\text {th }}$ percentiles and the median of $\mathrm{KBC}$ variables demonstrate that there is large withinindustry heterogeneity in the use of KBC. Across industries, the bottom decile of firms invest only little or nothing in $\mathrm{KBC}$, and consequently have very little or no KBC stocks. In services industries, the median $\mathrm{KBC}$ stocks and investments are also close to zero, while across manufacturing industries, the median values of KBC investment and stock are $€ 150,000$ and $€ 350,000$ respectively. Finally, the fact that the distribution of KBC is highly right-skewed is further visible in the fact that the average values of $\mathrm{KBC}$ stock and investment are higher than the $90^{\text {th }}$ percentile in all industries apart from Business Services.

Table 2 provides further information about the distribution of KBC stocks across detailed 2-digit industries. Important heterogeneity in the use of $\mathrm{KBC}$ is visible both across and within industries. In particular, we find that the bulk of $\mathrm{KBC}$ is concentrated in a few industries. The motor vehicle manufacturing industry (C29) stands out as having the highest average stock of KBC among all the industries in our analysis, at $€ 134$ million, followed by the pharmaceutical industry (C21) where the average stock of KBC stands at $€ 82$ million. The service industries with the largest average stock of $\mathrm{KBC}$ are broadcasting activities (J60) and telecommunications industries (J61), with an average stock of $€ 18$ and $€ 14$ million respectively.

The skewness of the within-industry distribution of $\mathrm{KBC}$ is further visible at the 2-digit level. Across industries, the bottom decile of firms report close to null KBC investments and stocks. Furthermore, the median stock of KBC reaches $€ 1$ million in only 3 industries, namely the chemical, pharmaceutical, and computer and electronics industries (C20, C21 and C26). In the services sector, the industries with the highest median stock of

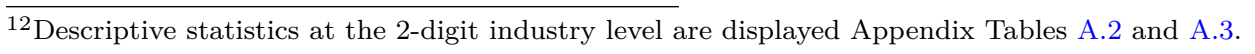


Table 1: Average production variables, by 1-digit industry

\begin{tabular}{|c|c|c|c|c|c|c|}
\hline Variable & 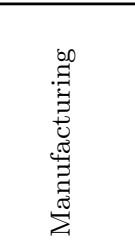 & 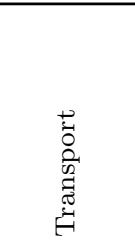 & 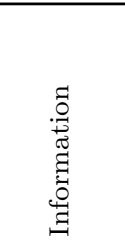 & $\begin{array}{l}0 \\
0 \\
.0 \\
D \\
0 \\
0 \\
0 \\
0 \\
0 \\
.7 \\
0 \\
0 \\
0\end{array}$ & 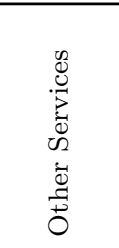 & 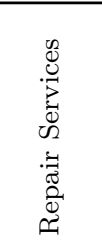 \\
\hline Value Added & $\begin{array}{c}21.42 \\
(220.24)\end{array}$ & $\begin{array}{c}3.91 \\
(78.74)\end{array}$ & $\begin{array}{c}6.83 \\
(112.82)\end{array}$ & $\begin{array}{c}1.79 \\
(15.4)\end{array}$ & $\begin{array}{c}2.73 \\
(22.46)\end{array}$ & $\begin{array}{c}.48 \\
(2.35)\end{array}$ \\
\hline Employment $^{\dagger}$ & $\begin{array}{c}267.41 \\
(1998.61)\end{array}$ & $\begin{array}{c}74.51 \\
(1541.27)\end{array}$ & $\begin{array}{c}69.28 \\
(945.49)\end{array}$ & $\begin{array}{c}24.76 \\
(178.23)\end{array}$ & $\begin{array}{c}88.74 \\
(558.7)\end{array}$ & $\begin{array}{c}11.12 \\
(42.41)\end{array}$ \\
\hline Capital & $\begin{array}{c}40.61 \\
(378.63)\end{array}$ & $\begin{array}{c}21.31 \\
(755.81)\end{array}$ & $\begin{array}{c}13.48 \\
(463.33)\end{array}$ & $\begin{array}{c}2.04 \\
(38.02)\end{array}$ & $\begin{array}{c}8.4 \\
(172.45)\end{array}$ & $\begin{array}{l}.24 \\
(1.3)\end{array}$ \\
\hline Material & $\begin{array}{c}37.38 \\
(478.55)\end{array}$ & $\begin{array}{c}6.62 \\
(89.55)\end{array}$ & $\begin{array}{c}8.71 \\
(162.18)\end{array}$ & $\begin{array}{c}1.74 \\
(57.42)\end{array}$ & $\begin{array}{c}2.35 \\
(40.43)\end{array}$ & $\begin{array}{c}.56 \\
(2.98)\end{array}$ \\
\hline Wagebill & $\begin{array}{c}33.75 \\
(13.44)\end{array}$ & $\begin{array}{c}20.16 \\
(35.25)\end{array}$ & $\begin{array}{c}35.21 \\
(60.24)\end{array}$ & $\begin{array}{c}26.31 \\
(56.21)\end{array}$ & $\begin{array}{c}17.44 \\
(27.35)\end{array}$ & $\begin{array}{c}13.79 \\
(13.27)\end{array}$ \\
\hline KBC Stock & $\begin{array}{c}14.14 \\
(305.32)\end{array}$ & $\begin{array}{c}1 \\
(20.91)\end{array}$ & $\begin{array}{c}2.22 \\
(41.36)\end{array}$ & $\begin{array}{c}1.6 \\
(17.38)\end{array}$ & $\begin{array}{c}.46 \\
(7.14)\end{array}$ & $\begin{array}{c}.25 \\
(2.66)\end{array}$ \\
\hline $\mathrm{P} 10$ & 0 & 0 & 0 & 0 & 0 & 0 \\
\hline Median & .35 & 0 & .07 & .02 & .01 & 0 \\
\hline P 90 & 7.1 & .23 & 1.49 & 1.99 & .38 & .25 \\
\hline KBC Investment & $\begin{array}{c}3.37 \\
(70.15)\end{array}$ & $\begin{array}{c}.23 \\
(4.96)\end{array}$ & $\begin{array}{c}.56 \\
(14.92)\end{array}$ & $\begin{array}{c}.36 \\
(4.21)\end{array}$ & $\begin{array}{c}.11 \\
(1.62)\end{array}$ & $\begin{array}{l}06 \\
.06)\end{array}$ \\
\hline P 10 & 0 & 0 & 0 & 0 & 0 & 0 \\
\hline Median & .15 & 0 & .02 & 0 & 0 & 0 \\
\hline P 90 & 2.21 & .07 & .33 & .44 & .12 & .05 \\
\hline KBC Intensity ${ }^{\ddagger}$ & $\begin{array}{l}.11 \\
(.36)\end{array}$ & $\begin{array}{c}.02 \\
(.61)\end{array}$ & $\begin{array}{c}.9 \\
(35.12)\end{array}$ & $\begin{array}{c}1.56 \\
(22.86)\end{array}$ & $\begin{array}{c}1.14 \\
(195.47)\end{array}$ & $\begin{array}{c}.68 \\
(2.83)\end{array}$ \\
\hline P 10 & 0 & 0 & .01 & 0 & 0 & 0 \\
\hline Median & .04 & 0 & .16 & .06 & .03 & .04 \\
\hline P 90 & .27 & .02 & 1.3 & 3.98 & .32 & 1.44 \\
\hline $\mathrm{N}$ & 87325 & 178903 & 111789 & 350596 & 188702 & 13513 \\
\hline
\end{tabular}

Standard deviation in parentheses. Monetary values in million $€$. $^{\dagger}$ : head-count number of employees. ${ }^{\ddagger}$ : KBC Intensity is defined as the the ratio of KBC stock over physical capital stock. 
Table 2: Percentiles and mean of $\mathrm{KBC}$ stocks and investment by 2-digit industry

\begin{tabular}{|c|c|c|c|c|c|c|c|c|c|c|}
\hline & \multicolumn{5}{|c|}{ KBC Stock } & \multicolumn{5}{|c|}{ KBC Investment } \\
\hline & P 10 & Median & P 90 & P 95 & Mean & P 10 & Median & P 90 & P 95 & Mean \\
\hline $\mathrm{C} 10$ & 0.000 & 0.25 & 2.28 & 4.81 & 1.36 & 0.000 & 0.13 & 0.96 & 1.88 & 0.51 \\
\hline C11 & 0.005 & 0.32 & 2.43 & 5.06 & 1.57 & 0.000 & 0.15 & 1.01 & 2.20 & 0.65 \\
\hline $\mathrm{C} 13$ & 0.008 & 0.29 & 2.30 & 4.00 & 1.14 & 0.000 & 0.11 & 0.81 & 1.31 & 0.35 \\
\hline C14 & 0.000 & 0.15 & 2.72 & 4.91 & 1.17 & 0.000 & 0.05 & 0.94 & 1.72 & 0.43 \\
\hline $\mathrm{C} 15$ & 0.000 & 0.14 & 1.74 & 3.34 & 0.78 & 0.000 & 0.05 & 0.61 & 0.97 & 0.27 \\
\hline $\mathrm{C} 16$ & 0.000 & 0.13 & 1.30 & 2.67 & 0.58 & 0.000 & 0.06 & 0.50 & 0.98 & 0.21 \\
\hline $\mathrm{C} 17$ & 0.059 & 0.41 & 3.62 & 6.85 & 1.56 & 0.031 & 0.17 & 1.29 & 2.18 & 0.55 \\
\hline C18 & 0.000 & 0.20 & 1.44 & 2.48 & 0.99 & 0.000 & 0.09 & 0.60 & 1.03 & 0.33 \\
\hline $\mathrm{C} 20$ & 0.102 & 1.01 & 15.69 & 34.74 & 23.11 & 0.040 & 0.31 & 4.21 & 8.31 & 5.27 \\
\hline $\mathrm{C} 21$ & 0.113 & 1.59 & 42.94 & 216.29 & 81.78 & 0.039 & 0.55 & 10.82 & 50.53 & 17.57 \\
\hline $\mathrm{C} 22$ & 0.000 & 0.27 & 5.55 & 15.65 & 4.31 & 0.000 & 0.12 & 1.77 & 4.08 & 1.10 \\
\hline $\mathrm{C} 23$ & 0.018 & 0.42 & 5.15 & 10.27 & 2.51 & 0.000 & 0.18 & 1.75 & 3.17 & 0.77 \\
\hline $\mathrm{C} 24$ & 0.000 & 0.27 & 5.14 & 11.47 & 3.43 & 0.000 & 0.12 & 1.57 & 3.13 & 0.88 \\
\hline $\mathrm{C} 25$ & 0.000 & 0.22 & 3.07 & 7.43 & 2.13 & 0.000 & 0.12 & 1.15 & 2.50 & 0.65 \\
\hline $\mathrm{C} 26$ & 0.091 & 1.61 & 32.01 & 72.37 & 23.21 & 0.030 & 0.45 & 7.91 & 17.92 & 5.27 \\
\hline $\mathrm{C} 27$ & 0.040 & 0.41 & 14.55 & 38.74 & 24.16 & 0.018 & 0.14 & 3.74 & 8.72 & 5.25 \\
\hline $\mathrm{C} 28$ & 0.047 & 0.53 & 15.50 & 43.75 & 15.38 & 0.023 & 0.20 & 4.29 & 10.95 & 3.80 \\
\hline $\mathrm{C} 29$ & 0.008 & 0.52 & 36.57 & 107.51 & 133.56 & 0.000 & 0.19 & 8.63 & 26.51 & 31.30 \\
\hline C30 & 0.002 & 0.43 & 23.53 & 127.15 & 55.49 & 0.000 & 0.14 & 5.28 & 34.16 & 12.31 \\
\hline C31 & 0.062 & 0.23 & 2.87 & 6.48 & 2.55 & 0.026 & 0.10 & 1.00 & 2.25 & 0.73 \\
\hline C32 & 0.000 & 0.24 & 7.76 & 23.26 & 6.04 & 0.000 & 0.11 & 2.48 & 6.80 & 1.74 \\
\hline C33 & 0.000 & 0.27 & 2.38 & 5.38 & 3.52 & 0.000 & 0.13 & 1.00 & 2.20 & 0.86 \\
\hline H49 & 0.000 & 0.00 & 0.12 & 0.28 & 0.64 & 0.000 & 0.00 & 0.04 & 0.10 & 0.14 \\
\hline H50 & 0.000 & 0.00 & 0.04 & 0.58 & 0.49 & 0.000 & 0.00 & 0.00 & 0.09 & 0.11 \\
\hline H51 & 0.000 & 0.00 & 4.58 & 23.25 & 6.37 & 0.000 & 0.00 & 0.98 & 4.51 & 1.38 \\
\hline H52 & 0.000 & 0.03 & 0.81 & 2.30 & 1.86 & 0.000 & 0.01 & 0.23 & 0.58 & 0.42 \\
\hline H53 & 0.000 & 0.00 & 0.17 & 0.42 & 0.59 & 0.000 & 0.00 & 0.06 & 0.12 & 0.23 \\
\hline J58 & 0.001 & 0.10 & 2.35 & 5.64 & 1.32 & 0.000 & 0.03 & 0.57 & 1.31 & 0.31 \\
\hline J59 & 0.000 & 0.02 & 0.36 & 1.13 & 0.55 & 0.000 & 0.01 & 0.10 & 0.25 & 0.13 \\
\hline J60 & 0.003 & 0.11 & 5.47 & 32.97 & 18.35 & 0.000 & 0.02 & 1.10 & 10.07 & 3.76 \\
\hline J61 & 0.000 & 0.05 & 2.17 & 9.54 & 14.18 & 0.000 & 0.01 & 0.44 & 1.94 & 3.90 \\
\hline J62 & 0.008 & 0.09 & 1.47 & 3.95 & 1.47 & 0.002 & 0.02 & 0.33 & 0.88 & 0.39 \\
\hline J63 & 0.001 & 0.04 & 1.17 & 3.28 & 1.23 & 0.000 & 0.01 & 0.22 & 0.68 & 0.23 \\
\hline M69 & 0.000 & 0.00 & 0.01 & 0.07 & 0.46 & 0.000 & 0.00 & 0.00 & 0.01 & 0.10 \\
\hline M70 & 0.009 & 0.46 & 8.70 & 19.06 & 5.28 & 0.001 & 0.10 & 1.91 & 4.14 & 1.20 \\
\hline M71 & 0.022 & 0.34 & 3.21 & 6.08 & 1.92 & 0.004 & 0.07 & 0.71 & 1.36 & 0.44 \\
\hline M72 & 0.001 & 0.03 & 0.56 & 1.11 & 0.37 & 0.000 & 0.01 & 0.19 & 0.37 & 0.13 \\
\hline M73 & 0.000 & 0.27 & 2.73 & 4.78 & 1.29 & 0.000 & 0.06 & 0.62 & 1.13 & 0.29 \\
\hline M74 & 0.000 & 0.00 & 0.10 & 0.37 & 0.43 & 0.000 & 0.00 & 0.02 & 0.06 & 0.10 \\
\hline M75 & 0.000 & 0.00 & 0.00 & 0.01 & 0.01 & 0.000 & 0.00 & 0.00 & 0.00 & 0.00 \\
\hline N77 & 0.000 & 0.00 & 0.69 & 1.55 & 0.72 & 0.000 & 0.00 & 0.15 & 0.34 & 0.17 \\
\hline N78 & 0.000 & 0.05 & 0.36 & 0.72 & 0.46 & 0.000 & 0.01 & 0.13 & 0.23 & 0.09 \\
\hline N79 & 0.000 & 0.01 & 0.09 & 0.24 & 0.49 & 0.000 & 0.00 & 0.03 & 0.07 & 0.11 \\
\hline N80 & 0.001 & 0.04 & 0.56 & 1.10 & 0.27 & 0.000 & 0.01 & 0.21 & 0.43 & 0.10 \\
\hline N81 & 0.000 & 0.00 & 0.25 & 0.51 & 0.13 & 0.000 & 0.00 & 0.09 & 0.20 & 0.05 \\
\hline N82 & 0.000 & 0.02 & 0.88 & 2.89 & 1.01 & 0.000 & 0.00 & 0.19 & 0.56 & 0.25 \\
\hline S95 & 0.000 & 0.00 & 0.25 & 0.96 & 0.25 & 0.000 & 0.00 & 0.05 & 0.20 & 0.06 \\
\hline
\end{tabular}

Monetary values in million $€$. 
KBC are management consultancy, architectural and engineering, and advertising and market research activities (M70, M71 and M73), with stocks of $€ 460,000, € 340,000$ and $€ 270,000$, respectively.

Finally, Table 2 also reports the value of KBC stocks and investment at the $95^{\text {th }}$ percentile, which delimits the set of firms on the KBC frontier in the analysis of Section 6. The pharmaceutical, motor vehicle, and other transport equipment industries (C21, C29 and C30) stand out, as the firms on the KBC frontier have stocks exceeding $€ 100$ million. Among the other high-tech manufacturing industries ${ }^{13}$, firms on the $\mathrm{KBC}$ frontier have stocks exceeding $€ 20$ million. In the services sector, air transport, broadcasting activities, and management consultancies (H51, J60 and M70) have KBC stocks above $€ 20$ million on the KBC frontier. On the contrary, in 10 service industries, the stock of $\mathrm{KBC}$ on the frontier does not reach $€ 1$ million.

\section{Methodology}

The present analysis is centred around firm-level productivity, both in its relationship to KBC within the firm and in its aggregate evolution. As first emphasised by Marschak and Andrews Jr. (1944), recovering productivity from production data is hampered by an inherent endogeneity problem, as firms have information on their productivity level when they make production decisions. From the econometric perspective, this unobserved factor, which is positively correlated with inputs, especially flexible inputs, and output, will introduce bias in the estimations.

To address this issue, we implement the control function approach pioneered by Olley and Pakes (1996) and further developed by Levinsohn and Petrin (2003) and Ackerberg et al. (2015). We specify a structural model of production, where the different inputs of production have different adjustments costs. In particular, we model intermediary inputs to have no adjustments costs, which implies that firms decide upon this expenditure after having observed productivity. The variation in this input can thus be used to control for variation in productivity. Furthermore, we acknowledge the impact of the firm's total stock of KBC on productivity in the model by including it in the law of motion of productivity.

Below, we present the details of our theoretical model and the estimation strategy used to recover firm-level productivity.

\subsection{Model of production}

Our model consists of two functions: a production function, as spelled out in Equation (1), which dictates how firms transform inputs into output; and the law of motion of productivity, spelled out in Equation (2), which dictates how productivity evolves through time.

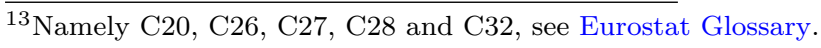


Firstly, we assume that firms produce according to a value-added production function of the form

$$
y_{i t}=f\left(l_{i t}, k_{i t} ; \boldsymbol{\beta}\right)+\underbrace{\varepsilon_{i t}}_{\omega_{i t}+\nu_{i t}}
$$

where $y_{i t}$ is value-added, $l_{i t}$ is labour input, and $k_{i t}$ is the stock of physical capital, and all variables are in logs. We allow for two different types of unobservables to affect the production function, subsumed in the error term $\varepsilon_{i t}$. Firstly, $\omega_{i t}$ is the firm's productivity, which we are interested in recovering. Secondly, $\nu_{i t}$ is a mean-zero i.i.d shock, which picks up measurement error or shocks to production, and is unanticipated by firms when they make their production decisions.

Secondly, we follow the literature and assume the evolution of productivity $\omega_{i t}$ to be governed by a first-order Markov process (Olley and Pakes, 1996; Levinsohn and Petrin, 2003; Ackerberg et al., 2015; De Loecker and Warzynski, 2012; Doraszelski and Jaumandreu, 2013; Ackerberg et al., 2015):

$$
\omega_{i t}=E\left[\omega_{i t} \mid \omega_{i t-1}, c_{i t-1}\right]+\xi_{i t}=g\left(\omega_{i t-1}, c_{i t-1}\right)+\xi_{i t}
$$

Realised productivity in period $t$ is composed of expected productivity $g(\cdot)$ and a random shock $\xi_{i t}$. Expected productivity has both exogenous and endogenous elements. The former reflects the decay of the previous period's productivity, and the latter takes into account the effect of the firm's decision to invest in KBC. In particular, we model today's productivity as dependent on last period's total stock of $\mathrm{KBC}, c_{i t-1}$, which is the sum of R\&D capital, software, intellectual property products, and organisational capital. Finally, the productivity shock $\xi_{i t}$ is unanticipated but observed by firms when they make their production decisions in period $t$.

The estimation strategy detailed below will allow us to recover the production coefficients $\beta$ and the conditional expectation function $g(\cdot)$.

\subsection{Estimation of the production function}

We follow the identification strategy of Ackerberg et al. (2015), whereby firms choose the level of intermediary inputs that will maximise static, or short-run, profits. The identification of the model is thus determined by the specification of the demand for intermediary inputs $m_{i t}$. As the most flexible input of production, the demand for $m_{i t}$ depends on the firm's state variables, which are labour, physical capital and KBC, all decided in the previous period and entering into operation in the current period given the adjustment costs, the realised productivity of period $t$, and the firm-specific average wage, $\mathrm{w}_{i t}$, (used as a proxy for all input prices). ${ }^{14}$ The

\footnotetext{
${ }^{14}$ See De Loecker and Warzynski (2012) for an extended discussion of the relevant variables to include in the demand for intermediary inputs, such as firm-specific input prices.
} 
intermediate input demand function can thus be expressed as follows

$$
m_{i t}=h_{t}\left(\omega_{i t}, l_{i t}, k_{i t}, c_{i t}, \mathrm{w}_{i t}\right)
$$

We further assume that productivity is the only unobservable that enters the demand for intermediary inputs and does so monotonically. The function $h(\cdot)$ can thus be inverted to obtain an expression for $\omega_{i t}$ as a function of observables ${ }^{15}$

$$
\omega_{i t}=h_{t}^{-1}\left(m_{i t}, l_{i t}, k_{i t}, c_{i t}, \mathrm{w}_{i t}\right)
$$

Productivity $\omega_{i t}$ is recovered using a two-step estimation procedure. The first stage allows us to net out the effect of the shock to production $\nu_{i t}$ by controlling for productivity with the proxy function $h_{t}^{-1}(\cdot)$. The second stage allows us to identify the unbiased production coefficients $\beta$, and to recover productivity.

Estimation of the first stage. In the first stage, we substitute $\omega_{i t}$ in the production function (1) by its expression in (4). We add further controls in the production function measuring the legal status of the firm, its geographical location, and time fixed effects, collected in the vector of control variables $\mathbf{X}_{i t}$. Our first stage estimation equation becomes

$$
\begin{aligned}
y_{i t} & =f\left(l_{i t}, k_{i t} ; \boldsymbol{\beta}\right)+h_{t}^{-1}\left(m_{i t}, l_{i t}, k_{i t}, c_{i t}, \mathrm{w}_{i t}\right)+\mathbf{X}_{i t} \gamma+\varepsilon_{i t} \\
& =\varphi_{i t}\left(m_{i t}, l_{i t}, k_{i t}, c_{i t}, \mathrm{w}_{i t}\right)+\mathbf{X}_{i t} \gamma+\varepsilon_{i t}
\end{aligned}
$$

We do not impose a functional form assumption on $h_{t}^{-1}(\cdot)$, and because the labour and capital inputs enter both the production function $f(\cdot)$ and the function $h_{t}^{-1}(\cdot)$, we cannot consistently estimate the production coefficients $\boldsymbol{\beta}$ in this first stage. ${ }^{16}$ We therefore estimate the generic function $\varphi_{i t}($.$) with a second-degree polynomial$ approximation, and predict $\hat{\varphi}_{i t}$. It follows from equations (4) and (5) that $\omega_{i t}$ can be predicted, up to the still unknown production coefficients, as:

$$
\tilde{\omega}_{i t}=\hat{\varphi}_{i t}\left(m_{i t}, l_{i t}, k_{i t}, c_{i t}, \mathrm{w}_{i t}\right)-f\left(l_{i t}, k_{i t} ; \boldsymbol{\beta}\right)
$$

Estimation of the second stage. Equation (6) is used to recover the production coefficients $\beta$ in the second stage of the estimation. We substitute unobserved productivity in Equation (2) with its expression in Equation (6) to

${ }^{15}$ See Levinsohn and Petrin (2003) for the proof of invertability.

${ }^{16}$ For an extensive discussion on this issue see Ackerberg et al. (2015). 
get

$$
\begin{aligned}
\tilde{\omega}_{i t} & =g\left(\tilde{\omega}_{i t-1}, c_{i t-1}\right)+u_{i t} \\
\hat{\varphi}_{i t}(\cdot)-f\left(l_{i t}, k_{i t} ; \boldsymbol{\beta}\right) & =g\left(\hat{\varphi}_{i t-1}(\cdot)-f\left(l_{i t-1}, k_{i t-1} ; \boldsymbol{\beta}\right), c_{i t-1}\right)+u_{i t}
\end{aligned}
$$

where $u_{i t}$ is the error term of this expression. It includes the unanticipated shock to productivity $\xi_{i t}$ and an i.i.d. error that captures measurement error resulting from the fact that we use the predicted values of $\hat{\varphi}_{i t}$. While observed by firms in $t$, this error term is uncorrelated with past productivity and the past stock of $\mathrm{KBC}$, and is used to build a GMM estimator to obtain the production coefficients $\boldsymbol{\beta}$.

The moment condition is based on the assumption that the expectation of $u_{i t}$, conditional on the information set in the previous year, $I_{i t-1}$, is equal to zero:

$$
\begin{aligned}
& E\left[u_{i t} \mid I_{i t-1}\right]=0 \\
& E\left[\tilde{\omega}_{i t}-g\left(\tilde{\omega}_{i t-1}, c_{i t-1}\right) \mid I_{i t-1}\right]=0
\end{aligned}
$$

The timing assumptions of our model are used to choose a vector of instruments $\boldsymbol{Z}_{i t}$ that satisfies

$$
E\left[u_{i t} \boldsymbol{Z}_{i t}\right]=0
$$

It contains the contemporaneous values of the labour and capital inputs and of KBC stock. The use of current physical capital stock stems from the assumption, widespread in the literature, that investment conducted in $t$ is decided upon in $t-1$ after $\xi_{i t-1}$ has been observed. Current capital stock is theretofore uncorrelated with the productivity shock in $t$. If labour has high adjustments costs, as it does in countries with rigid employment protection legislation (EPL), its value in $t$ will also be uncorrelated with the productivity shock $\xi_{i t} .{ }^{17}$ Finally, we extend the assumption found in the literature concerning the relationship between $R \& D$ and productivity to hold for all KBC assets (Aw et al., 2011; Doraszelski and Jaumandreu, 2013; Kancs and Siliverstovs, 2016). If a firm decides to invest in $\mathrm{R} \& \mathrm{D}$ and conducts the investment in year $t$, the effect on productivity will only be witnessed in $t+1$. We impose the same assumption on the other KBC assets, and consequently, assume that $c_{i t-1}$ is uncorrelated with $\xi_{i t}$.

Functional form assumptions. For our econometric analysis, we need to make functional form assumptions for both $f(\cdot)$ and $g(\cdot)$. Our preferred specification assumes a Cobb-Douglas production function for Equation (1)

\footnotetext{
${ }^{17}$ Ackerberg et al. (2015) discuss the conditions under which labour can be considered a fixed input, and argue that this is the case
} in countries with stringent EPL. See (OECD, 2015, p.64) for evidence for the rigidity of the German labor market. 
and a translog specification for the law of motion of Equation (7)

$$
\begin{aligned}
& y_{i t}=\beta_{l} l_{i t}+\beta_{k} k_{i t}+\omega_{i t}+\nu_{i t} \\
& \omega_{i t}=\beta_{\omega} \omega_{i t-1}+\beta_{\omega^{2}} \omega_{i t-1}^{2}+\beta_{c} c_{i t-1}+\beta_{c^{2}} c_{i t-1}^{2}+\beta_{c \omega} c_{i t-1} \omega_{i t-1}+u_{i t}
\end{aligned}
$$

We make a parsimonious assumption for the production function for the following reasons. Firstly, our main objective at this stage is to obtain stable estimates of the output elasticities of the production factors, and to recover unobserved firm productivity. We do not place much emphasis on the interpretation of these coefficients, beyond ensuring that we obtain values consistent with the literature. Secondly, this is also the assumption made by Andrews et al. (2019) and thereby allows us to directly compare our results to theirs.

The focus of our research question lies on the law of motion of productivity, and for this relationship, we assume a flexible functional form for the relationship between productivity and KBC stocks. In particular, this assumption allows us to recover firm- and time-specific marginal effects of past KBC and past productivity on current productivity ${ }^{18}$, according to

$$
\begin{aligned}
& \theta_{c_{i t-1}}=\frac{\partial \omega_{i t}}{\partial c_{i t-1}}=\beta_{c}+2 \beta_{c^{2}} c_{i t-1}+\beta_{c \omega} \omega_{i t-1} \\
& \theta_{\omega_{i t-1}}=\frac{\partial \omega_{i t}}{\partial \omega_{i t-1}}=\beta_{\omega}+2 \beta_{\omega^{2}} \omega_{i t-1}+\beta_{c \omega} c_{i t-1}
\end{aligned}
$$

In the remainder of the paper, we focus on the law of motion of productivity. The discussion around the production function $f(\cdot)$ is reported in Appendix B, where we present the estimated production coefficients $\boldsymbol{\beta}$.

\section{Effect of KBC on firm-level productivity}

\subsection{Main estimation results}

The results relating to the relationship between a firm's stock of knowledge assets and its productivity, obtained from the estimation of the law of motion of productivity, are reported in Tables 3 and $4 .{ }^{19}$ In the first two columns, we report the mean industry values of $\theta_{c}$ and $\theta_{\omega}$, calculated from Equations (12a) and (12b), along with the standard error of these means in parentheses. $\theta_{c}$ is the elasticity of $\mathrm{KBC}$, describing the percentage change of productivity from increasing a firm's stock of $\mathrm{KBC}$ by $1 \%$. $\theta_{\omega}$ gives the persistence of productivity. In the following 5 columns, we report the point estimates of the coefficients in the law of motion of productivity

$\overline{{ }^{18} \text { More generally, the output elasticity of any input }} \Psi$ for a translog function with $N$ variables is derived as follows

$$
\theta_{\psi i t}=\frac{\partial Y_{i t} / Y_{i t}}{\partial \Psi_{i t} / \Psi_{i t}}=\beta_{\psi}+2 \beta_{\psi^{2}} \psi_{i t}+\sum_{n \neq \psi}^{N} \beta_{\psi n} n_{i t}
$$

It measures the percentage change in the dependent variable resulting from a percentage change in the input $n$.

${ }^{19}$ Throughout, we report results for the manufacturing and services sectors separately, given their different production structures and the different way in which R\&D expenditures are measured. 
(excluding the constant term), and in the eighth column, we report the F-statistic for the test of joint significance of the three point estimates relating to $\mathrm{KBC}$, which are used in the calculation of $\theta_{c}$, namely $\beta_{c}, \beta_{c^{2}}$, and $\beta_{c \omega}$. Together with the standard error reported in parentheses, this gives an indication of the statistical significance of the calculated $\theta_{c}$.

In accordance with findings in the literature, the average effect of $\mathrm{KBC}$ on productivity is positive and significant across the economy. ${ }^{20}$ The magnitude of the effect differs between the manufacturing and services sectors. On average, across the manufacturing sectors, a $1 \%$ increase $\mathrm{KBC}$ stock is followed by $0.007 \%$ increase in productivity. Across the services sectors, this effect reaches $0.03 \%{ }^{21}$ This difference in magnitude can be driven in part by the different measure of $R \& D$ between these two sectors. The test of joint significance suggests that despite the small magnitudes, especially in the manufacturing sector, this effect is statistically significant.

More generally, there is a positive correlation between the effect of KBC on productivity and the observed investment behaviour of firms. $\theta_{c}$ is higher in industries with larger median $\mathrm{KBC}$ stocks. The correlation between $\theta_{c}$ and the median KBC stock is 0.41 in the manufacturing sector and 0.73 in the services sector. The correlation between $\theta_{c}$ and the mean $\mathrm{KBC}$ stock is much lower, at 0.15 in the manufacturing sector and 0.11 in the services sector. This might be driven by the important skewness of the KBC stock within industries. Furthermore, we find a strong negative correlation between $\theta_{c}$ and $\theta_{\omega}$, of -0.31 in the manufacturing sector and -0.75 in the services sectors, which implies that productivity displays less persistence in industries where the effect of KBC on productivity is higher.

The main hypothesis of this first stage of the analysis states that in addition to a positive average effect, there is heterogeneity across firms and increasing returns to the size of the KBC stock. In Tables 3 and 4 , we report the point estimates of the law of motion of productivity, to shed light on the existence of potential non-linearities in the relationship between KBC and productivity. Of particular interest are the coefficients on the squared term of $\mathrm{KBC}, \beta_{c^{2}}$, and on the interaction term between productivity and $\mathrm{KBC}, \beta_{c \omega}$. The former tells us whether there are increasing returns to the size of the KBC stock, and the latter whether the effect of $\mathrm{KBC}$ on productivity is augmented in more productive firms.

\footnotetext{
${ }^{20}$ The elasticity of $\mathrm{KBC}$ on productivity is negative in only 1 sector, beverages (C11), and is indistinguishable from zero in 2 sectors, wearing apparel and wood products (C14 and C16). These three sectors are often classified as low-tech manufacturing sectors.

${ }^{21}$ In the manufacturing industry, the magnitude ranges from a low of 0.001-0.003 in the manufacturing of basic metals (C24) and of other transport equipment (C30) to a high of 0.016 in paper products (C17), computer and electronics (C26) and in furniture (C31). In the services sector, the elasticity of KBC ranges from 0.004-0.006 in water transport (H50), legal and accounting services (M69) and veterinary activities (M75), to 0.1 in management consulting services (70), architectural and engineering design (M71), and security and investigation activities (N80).
} 
Table 3: Marginal effect of KBC on productivity and point estimates of law of motion of productivity, by 2-digit industry in Manufacturing

\begin{tabular}{|c|c|c|c|c|c|c|c|c|c|}
\hline \multirow[b]{2}{*}{ Industry } & \multicolumn{2}{|c|}{ Average } & \multicolumn{5}{|c|}{ Point estimates of the law of motion of productivity } & \multirow[b]{2}{*}{ F stat } & \multirow[b]{2}{*}{$\mathrm{N}$} \\
\hline & $\theta_{c}$ & $\theta_{\omega}$ & $\beta_{\omega}$ & $\beta_{\omega^{2}}$ & $\beta_{c}$ & $\beta_{c^{2}}$ & $\beta_{c \omega}$ & & \\
\hline $\mathrm{C} 10$ & $\begin{array}{c}.005 \\
(.00349)\end{array}$ & $\begin{array}{c}.907 \\
(.0221)\end{array}$ & $\begin{array}{c}.593^{* * *} \\
(.06)\end{array}$ & $\begin{array}{c}.03^{* * *} \\
(.01)\end{array}$ & $\begin{array}{c}.014^{* * *} \\
(.00)\end{array}$ & $\begin{array}{c}.0004^{* * *} \\
(.00)\end{array}$ & $\begin{array}{c}-.003^{* * *} \\
(.00)\end{array}$ & $34.79^{* * *}$ & 9482 \\
\hline $\mathrm{C} 11$ & $\begin{array}{c}-.004 \\
(.00262)\end{array}$ & $\begin{array}{c}.9 \\
(.0725)\end{array}$ & $\begin{array}{c}.257^{* * *} \\
(.1)\end{array}$ & $\begin{array}{c}.083^{* * *} \\
(.01)\end{array}$ & $\begin{array}{l}.021^{*} \\
(.01)\end{array}$ & $\begin{array}{c}-.0003^{*} \\
(.00)\end{array}$ & $\begin{array}{l}-.004 \\
(.00)\end{array}$ & $2.66^{* *}$ & 1297 \\
\hline $\mathrm{C} 13$ & $\begin{array}{c}.005 \\
(.00261)\end{array}$ & $\begin{array}{c}.92 \\
(.0264)\end{array}$ & $\begin{array}{c}1.549^{* * *} \\
(.18)\end{array}$ & $\begin{array}{c}-.037^{* * * *} \\
(.01)\end{array}$ & $\begin{array}{l}-.018 \\
(.02)\end{array}$ & $\begin{array}{c}.0004^{* *} \\
(.00)\end{array}$ & $\begin{array}{l}.002 \\
(.00)\end{array}$ & $2.99 * *$ & 1929 \\
\hline $\mathrm{C} 14$ & $\begin{array}{c}0 \\
(.00441)\end{array}$ & $\begin{array}{c}.955 \\
(.0304)\end{array}$ & $\begin{array}{c}.595^{* * *} \\
(.14)\end{array}$ & $\begin{array}{c}.028^{* * *} \\
(.01)\end{array}$ & $\begin{array}{c}.038^{* * * *} \\
(.01)\end{array}$ & $\begin{array}{c}-.0003 \\
(.00)\end{array}$ & $\begin{array}{c}-.004^{* *} \\
(.00)\end{array}$ & $4.92^{* * *}$ & 1053 \\
\hline $\mathrm{C} 15$ & $\begin{array}{c}.005 \\
(.00364)\end{array}$ & $\begin{array}{c}.922 \\
(.0395)\end{array}$ & $\begin{array}{c}.406^{* *} \\
(.2)\end{array}$ & $\begin{array}{c}.052^{* * *} \\
(.02)\end{array}$ & $\begin{array}{c}.02 \\
(.02)\end{array}$ & $\begin{array}{l}.0004 \\
(.00)\end{array}$ & $\begin{array}{c}-.004 \\
(.00)\end{array}$ & $2.15^{*}$ & 469 \\
\hline $\mathrm{C} 16$ & $\begin{array}{c}0 \\
(.0004)\end{array}$ & $\begin{array}{c}.909 \\
(.0309)\end{array}$ & $\begin{array}{c}1.686^{* * *} \\
(.27)\end{array}$ & $\begin{array}{c}-.053^{* * *} \\
(.02)\end{array}$ & $\begin{array}{l}.002 \\
(.01)\end{array}$ & $\begin{array}{c}.00 \\
(.00)\end{array}$ & $\begin{array}{c}.00 \\
(.00)\end{array}$ & .5 & 1994 \\
\hline $\mathrm{C} 17$ & $\begin{array}{c}.017 \\
(.00632)\end{array}$ & $\begin{array}{c}.907 \\
(.0279)\end{array}$ & $\begin{array}{c}.702^{* * *} \\
(.15)\end{array}$ & $\begin{array}{c}.032^{* *} \\
(.01)\end{array}$ & $\begin{array}{c}.127^{* * * *} \\
(.04)\end{array}$ & $\begin{array}{c}.0024^{* * *} \\
(.00)\end{array}$ & $\begin{array}{c}-.022^{* * *} \\
(.01)\end{array}$ & $29.24^{* * *}$ & 2257 \\
\hline $\mathrm{C} 18$ & $\begin{array}{c}.012 \\
(.0085)\end{array}$ & $\begin{array}{c}.899 \\
(.0179)\end{array}$ & $\begin{array}{c}1.264^{* * *} \\
(.29)\end{array}$ & $\begin{array}{l}-.021 \\
(.02)\end{array}$ & $\begin{array}{l}.00 \\
(.02)\end{array}$ & $\begin{array}{c}.001^{* * *} \\
(.00)\end{array}$ & $\begin{array}{l}-.001 \\
(.00)\end{array}$ & $17.81^{* * *}$ & 1635 \\
\hline $\mathrm{C} 20$ & $\begin{array}{c}.012 \\
(.00346)\end{array}$ & $\begin{array}{c}.925 \\
(.0154)\end{array}$ & $\begin{array}{c}.802^{* * *} \\
(.1)\end{array}$ & $\begin{array}{l}.015^{*} \\
(.01)\end{array}$ & $\begin{array}{c}.051^{* * *} \\
(.01)\end{array}$ & $\begin{array}{c}.0008^{* * *} \\
(.00)\end{array}$ & $\begin{array}{c}-.008^{* * *} \\
(.00)\end{array}$ & $35.98^{* * *}$ & 4222 \\
\hline $\mathrm{C} 21$ & $\begin{array}{c}.008 \\
(.00342)\end{array}$ & $\begin{array}{c}.887 \\
(.0504)\end{array}$ & $\begin{array}{l}.004 \\
(.22)\end{array}$ & $\begin{array}{c}.078^{* * *} \\
(.02)\end{array}$ & $\begin{array}{l}.068^{*} \\
(.04)\end{array}$ & $\begin{array}{l}.0004 \\
(.00)\end{array}$ & $\begin{array}{c}-.011^{*} \\
(.01)\end{array}$ & $4.32^{* * *}$ & 913 \\
\hline $\mathrm{C} 22$ & $\begin{array}{c}.006 \\
(.00307)\end{array}$ & $\begin{array}{c}.899 \\
(.002)\end{array}$ & $\begin{array}{c}.887^{* * *} \\
(.14)\end{array}$ & $\begin{array}{l}.001 \\
(.01)\end{array}$ & $\begin{array}{l}.001 \\
(.01)\end{array}$ & $\begin{array}{c}.0004^{* * *} \\
(.00)\end{array}$ & $\begin{array}{l}-.001 \\
(.00)\end{array}$ & $23.09^{* * *}$ & 4573 \\
\hline $\mathrm{C} 23$ & $\begin{array}{c}.009 \\
(.00486)\end{array}$ & $\begin{array}{c}.926 \\
(.0113)\end{array}$ & $\begin{array}{c}1.153^{* * *} \\
(.14)\end{array}$ & $\begin{array}{c}-.014 \\
(.01)\end{array}$ & $\begin{array}{r}-.007 \\
(.02)\end{array}$ & $\begin{array}{c}.0008^{* * *} \\
(.00)\end{array}$ & $\begin{array}{c}.00 \\
(.00)\end{array}$ & $18.66^{* * *}$ & 3763 \\
\hline $\mathrm{C} 24$ & $\begin{array}{c}.004 \\
(.00205)\end{array}$ & $\begin{array}{c}.858 \\
(.0145)\end{array}$ & $\begin{array}{c}.644^{* * *} * \\
(.24)\end{array}$ & $\begin{array}{l}.017 \\
(.02)\end{array}$ & $\begin{array}{c}.023^{* *} \\
(.01)\end{array}$ & $\begin{array}{c}.0003^{* * *} \\
(.00)\end{array}$ & $\begin{array}{c}-.003^{* *} \\
(.00)\end{array}$ & $12.01^{* * *}$ & 3275 \\
\hline $\mathrm{C} 25$ & $\begin{array}{c}.004 \\
(.0034)\end{array}$ & $\begin{array}{c}.878 \\
(.0149)\end{array}$ & $\begin{array}{c}1.295^{* * *} \\
(.14)\end{array}$ & $\begin{array}{c}-.027^{* * *} \\
(.01)\end{array}$ & $\begin{array}{c}-.021^{* * *} \\
(.01)\end{array}$ & $\begin{array}{c}.0003^{* * *} \\
(.00)\end{array}$ & $\begin{array}{c}.002^{* *} \\
(.00)\end{array}$ & $46.73^{* * *}$ & 10430 \\
\hline $\mathrm{C} 26$ & $\begin{array}{c}.016 \\
(.00563)\end{array}$ & $\begin{array}{c}.885 \\
(.0305)\end{array}$ & $\begin{array}{l}.108 \\
(.3)\end{array}$ & $\begin{array}{c}.042^{* * *} \\
(.01)\end{array}$ & $\begin{array}{c}.134^{* * *} \\
(.03)\end{array}$ & $\begin{array}{c}.0013^{* * *} \\
(.00)\end{array}$ & $\begin{array}{c}-.013^{* * *} \\
(.00)\end{array}$ & $46.52^{* * *}$ & 3664 \\
\hline $\mathrm{C} 27$ & $\begin{array}{c}.008 \\
(.00279)\end{array}$ & $\begin{array}{c}.892 \\
(.0246)\end{array}$ & $\begin{array}{c}1.131^{* * *} \\
(.13)\end{array}$ & $\begin{array}{r}-.008 \\
(.01)\end{array}$ & $\begin{array}{c}.059 * * * \\
(.02)\end{array}$ & $\begin{array}{c}.0006^{* * *} \\
(.00)\end{array}$ & $\begin{array}{c}-.007^{* * *} \\
(.00)\end{array}$ & $32.13^{* * *}$ & 5063 \\
\hline $\mathrm{C} 28$ & $\begin{array}{c}.007 \\
(.00174)\end{array}$ & $\begin{array}{c}.861 \\
(.0099)\end{array}$ & $\begin{array}{c}1.151^{* * *} \\
(.15)\end{array}$ & $\begin{array}{c}-.018^{* *} \\
(.01)\end{array}$ & $\begin{array}{r}-.023 \\
(.02)\end{array}$ & $\begin{array}{c}.0002^{* * *} \\
(.00)\end{array}$ & $\begin{array}{l}.003 \\
(.00)\end{array}$ & $77.09^{* * *}$ & 11500 \\
\hline $\mathrm{C} 29$ & $\begin{array}{c}.011 \\
(.00466)\end{array}$ & $\begin{array}{c}.897 \\
(.0273)\end{array}$ & $\begin{array}{c}.543^{* * *} * \\
(.12)\end{array}$ & $\begin{array}{c}.034^{* * *} \\
(.01)\end{array}$ & $\begin{array}{c}.047^{* * *} * \\
(.01)\end{array}$ & $\begin{array}{c}.0008^{* * *} \\
(.00)\end{array}$ & $\begin{array}{c}-.008^{* * *} \\
(.00)\end{array}$ & $25.84^{* * *}$ & 2974 \\
\hline C30 & $\begin{array}{c}.002 \\
(.0009)\end{array}$ & $\begin{array}{c}.928 \\
(.0112)\end{array}$ & $\begin{array}{l}.93^{* *} \\
(.44)\end{array}$ & $\begin{array}{l}.002 \\
(.03)\end{array}$ & $\begin{array}{l}.021 \\
(.02)\end{array}$ & $\begin{array}{l}.0001 \\
(.00)\end{array}$ & $\begin{array}{l}-.003 \\
(.00)\end{array}$ & 1.13 & 1000 \\
\hline C31 & $\begin{array}{l}.016 \\
(.0051)\end{array}$ & $\begin{array}{c}.853 \\
(.0179)\end{array}$ & $\begin{array}{l}.907^{*} \\
(.52)\end{array}$ & $\begin{array}{l}-.014 \\
(.04)\end{array}$ & $\begin{array}{l}-.042 \\
(.07)\end{array}$ & $\begin{array}{c}-.0021^{* * *} \\
(.00)\end{array}$ & $\begin{array}{l}.014 \\
(.01)\end{array}$ & $21.63^{* * *}$ & 1875 \\
\hline C32 & $\begin{array}{c}.006 \\
(.00505)\end{array}$ & $\begin{array}{c}.923 \\
(.0225)\end{array}$ & $\begin{array}{c}.42^{* * *} \\
(.15)\end{array}$ & $\begin{array}{c}.031^{* * *} \\
(.01)\end{array}$ & $\begin{array}{l}.017 \\
(.01)\end{array}$ & $\begin{array}{c}.0006^{* * *} \\
(.00)\end{array}$ & $\begin{array}{c}-.003^{*} \\
(.00)\end{array}$ & $19.66^{* * *}$ & 3042 \\
\hline C33 & $\begin{array}{c}.004 \\
(.00384)\end{array}$ & $\begin{array}{c}.907 \\
(.0177)\end{array}$ & $\begin{array}{l}.77^{* * *} \\
(.21)\end{array}$ & $\begin{array}{c}.01 \\
(.01)\end{array}$ & $\begin{array}{l}.034^{* *} \\
(.01)\end{array}$ & $\begin{array}{c}.0004^{* * *} \\
(.00)\end{array}$ & $\begin{array}{c}-.004^{* *} \\
(.00)\end{array}$ & $10.44^{* * *}$ & 3171 \\
\hline
\end{tabular}

*** $\mathrm{p}<0.01, * * \mathrm{p}<0.05, * \mathrm{p}<0.1$. Standard errors in parentheses. Point estimates of the constant term unreported. $\mathrm{F}$ statistic for the test of joint significance of the $3 \mathrm{KBC}$ terms $\left(\beta_{c}, \beta_{c^{2}}\right.$ and $\left.\beta_{c \omega}\right)$ 
Table 4: Marginal effect of KBC on productivity and point estimates of law of motion of productivity, by 2-digit industry in Services

\begin{tabular}{|c|c|c|c|c|c|c|c|c|c|}
\hline \multirow[b]{2}{*}{ Industry } & \multicolumn{2}{|c|}{ Average } & \multicolumn{5}{|c|}{ Point estimates of the law of motion of productivity } & \multirow[b]{2}{*}{ F stat } & \multirow[b]{2}{*}{$\mathrm{N}$} \\
\hline & $\theta_{c}$ & $\theta_{\omega}$ & $\beta_{\omega}$ & $\beta_{\omega^{2}}$ & $\beta_{c}$ & $\beta_{c^{2}}$ & $\beta_{c \omega}$ & & \\
\hline H49 & $\begin{array}{c}.008 \\
(.00161)\end{array}$ & $\begin{array}{c}.844 \\
(.0117)\end{array}$ & $\begin{array}{c}.861^{* * *} \\
(.02)\end{array}$ & $\begin{array}{c}-.003^{*} \\
(.00)\end{array}$ & $\begin{array}{c}-.005^{* * *} \\
(.00)\end{array}$ & $\begin{array}{l}.0001 \\
(.00)\end{array}$ & $\begin{array}{c}.002 * * * \\
(.00)\end{array}$ & $733.89^{* * *}$ & 75957 \\
\hline $\mathrm{H} 50$ & $\begin{array}{c}.005 \\
(.00766)\end{array}$ & $\begin{array}{c}.86 \\
(.0798)\end{array}$ & $\begin{array}{c}1.662^{* * * *} \\
(.07)\end{array}$ & $\begin{array}{c}-.045^{* * *} \\
(.00)\end{array}$ & $\begin{array}{l}.005 \\
(.01)\end{array}$ & $\begin{array}{c}.0008^{* * *} \\
(.00)\end{array}$ & $\begin{array}{r}-.001 \\
(.00)\end{array}$ & $31.88^{* * *}$ & 7454 \\
\hline H51 & $\begin{array}{c}.017 \\
(.01497)\end{array}$ & $\begin{array}{c}.803 \\
(.1267)\end{array}$ & $\begin{array}{c}1.987^{* * *} \\
(.06)\end{array}$ & $\begin{array}{c}-.068^{* * *} \\
(.00)\end{array}$ & $\begin{array}{l}.003 \\
(.02)\end{array}$ & $\begin{array}{c}.0012^{* * *} \\
(.00)\end{array}$ & $\begin{array}{l}.00 \\
(.00)\end{array}$ & $39.81^{* * *}$ & 1482 \\
\hline $\mathrm{H} 52$ & $\begin{array}{c}.022 \\
(.01277)\end{array}$ & $\begin{array}{l}.825 \\
(.026)\end{array}$ & $\begin{array}{c}1.015^{* * *} \\
(.05)\end{array}$ & $\begin{array}{c}-.008^{* * *} \\
(.00)\end{array}$ & $\begin{array}{c}.029 * * * \\
(.01)\end{array}$ & $\begin{array}{c}.0015^{* * *} \\
(.00)\end{array}$ & $\begin{array}{c}-.004^{* * *} \\
(.00)\end{array}$ & $603.11^{* * *}$ & 32179 \\
\hline $\mathrm{H} 53$ & $\begin{array}{c}.007 \\
(.00469)\end{array}$ & $\begin{array}{l}.899 \\
(.03)\end{array}$ & $\begin{array}{c}.866^{* * *} \\
(.06)\end{array}$ & $\begin{array}{l}.00 \\
(.00)\end{array}$ & $\begin{array}{c}-.032^{* * *} \\
(.01)\end{array}$ & $\begin{array}{l}.0001 \\
(.00)\end{array}$ & $\begin{array}{c}.005^{* * *} \\
(.00)\end{array}$ & $54.26^{* * *}$ & 7820 \\
\hline $\mathrm{J} 58$ & $\begin{array}{c}.013 \\
(.00916)\end{array}$ & $\begin{array}{c}.897 \\
(.0473)\end{array}$ & $\begin{array}{c}1.382^{* * *} \\
(.03)\end{array}$ & $\begin{array}{c}-.032^{* * * *} \\
(.00)\end{array}$ & $\begin{array}{l}.003 \\
.01)\end{array}$ & $\begin{array}{c}.0013^{* * *} \\
(.00)\end{array}$ & $\begin{array}{c}-.002^{* * *} \\
(.00)\end{array}$ & $61.23^{* * *}$ & 10931 \\
\hline J59 & $\begin{array}{c}.021 \\
(.01192)\end{array}$ & $\begin{array}{c}.894 \\
(.0202)\end{array}$ & $\begin{array}{c}1.121^{* * *} \\
(.08)\end{array}$ & $\begin{array}{c}-.015^{* * *} \\
(.01)\end{array}$ & $\begin{array}{c}-.033^{* * *} \\
(.01)\end{array}$ & $\begin{array}{c}.0012^{* * *} \\
(.00)\end{array}$ & $\begin{array}{c}.004^{* *} \\
(.00)\end{array}$ & $61.08^{* * *}$ & 5869 \\
\hline $\mathrm{J} 60$ & $\begin{array}{l}.037 \\
(.0144)\end{array}$ & $\begin{array}{c}.863 \\
(.0189)\end{array}$ & $\begin{array}{c}.778^{* * *} \\
(.09)\end{array}$ & $\begin{array}{l}.009 \\
(.01)\end{array}$ & $\begin{array}{l}.026 \\
(.04)\end{array}$ & $\begin{array}{c}.0023^{* *} \\
(.00)\end{array}$ & $\begin{array}{l}-.005 \\
(.01)\end{array}$ & $12.27 * * *$ & 838 \\
\hline J61 & $\begin{array}{c}.025 \\
(.00752)\end{array}$ & $\begin{array}{l}.866 \\
(.03)\end{array}$ & $\begin{array}{c}.587^{* * *} \\
(.07)\end{array}$ & $\begin{array}{c}.021^{* * *} \\
(.01)\end{array}$ & $\begin{array}{l}.023^{*} \\
(.01)\end{array}$ & $\begin{array}{c}.0011^{* * *} \\
(.00)\end{array}$ & $\begin{array}{r}-.003 \\
(.00)\end{array}$ & $48.04^{* * *}$ & 4212 \\
\hline J62 & $\begin{array}{c}.028 \\
(.00819)\end{array}$ & $\begin{array}{c}.778 \\
(.0123)\end{array}$ & $\begin{array}{c}.941^{* * *} \\
(.06)\end{array}$ & $\begin{array}{c}-.013^{* * *} \\
(.00)\end{array}$ & $\begin{array}{c}.027^{* *} \\
(.01)\end{array}$ & $\begin{array}{c}-.0022^{* * *} \\
(.00)\end{array}$ & $\begin{array}{c}.006^{* * *} \\
(.00)\end{array}$ & $537.68^{* * *}$ & 43097 \\
\hline J63 & $\begin{array}{c}.019 \\
(.01788)\end{array}$ & $\begin{array}{c}.832 \\
(.0636)\end{array}$ & $\begin{array}{c}1.52^{* * *} \\
(.05)\end{array}$ & $\begin{array}{c}-.046^{* * *} \\
(.00)\end{array}$ & $\begin{array}{c}-.105^{* * *} \\
(.01)\end{array}$ & $\begin{array}{c}.0021^{* * *} \\
(.00)\end{array}$ & $\begin{array}{c}.009^{* * *} \\
(.00)\end{array}$ & $137.18^{* * *}$ & 10904 \\
\hline M69 & $\begin{array}{c}.007 \\
(.00214)\end{array}$ & $\begin{array}{c}.826 \\
(.0079)\end{array}$ & $\begin{array}{c}.955^{* * *} \\
(.03)\end{array}$ & $\begin{array}{c}-.01^{* * *} \\
(.00)\end{array}$ & $\begin{array}{l}.002 \\
(.00)\end{array}$ & $\begin{array}{c}-.0002^{* * *} \\
(.00)\end{array}$ & $\begin{array}{c}.001^{* *} \\
(.00)\end{array}$ & $438.44^{* * *}$ & 81249 \\
\hline M70 & $\begin{array}{c}.102 \\
(.03484)\end{array}$ & $\begin{array}{c}.725 \\
(.0392)\end{array}$ & $\begin{array}{c}.375^{* * *} * \\
(.04)\end{array}$ & $\begin{array}{c}.029^{* * * *} \\
(.00)\end{array}$ & $\begin{array}{c}.116^{* * *} \\
(.01)\end{array}$ & $\begin{array}{c}.0077^{* * *} * \\
(.00)\end{array}$ & $\begin{array}{c}-.02^{* * *} \\
(.00)\end{array}$ & $1555.58^{* * *}$ & 32538 \\
\hline M71 & $\begin{array}{c}.113 \\
(.03496)\end{array}$ & $\begin{array}{c}.669 \\
(.0603)\end{array}$ & $\begin{array}{c}-.656^{* * *} \\
(.05)\end{array}$ & $\begin{array}{c}.117^{* * * *} \\
(.00)\end{array}$ & $\begin{array}{c}.382^{* * * *} \\
(.02)\end{array}$ & $\begin{array}{c}.0205^{* * *} \\
(.00)\end{array}$ & $\begin{array}{c}-.079^{* * *} \\
(.00)\end{array}$ & $3833.25^{* * *}$ & 59139 \\
\hline M72 & $\begin{array}{l}.027 \\
(.0097)\end{array}$ & $\begin{array}{c}.829 \\
(.0614)\end{array}$ & $\begin{array}{c}-.236^{*} \\
(.12)\end{array}$ & $\begin{array}{c}.071^{* * *} \\
(.01)\end{array}$ & $\begin{array}{c}.15^{* * * *} \\
(.02)\end{array}$ & $\begin{array}{c}.002^{* * *} \\
(.00)\end{array}$ & $\begin{array}{c}-.019^{* * *} \\
(.00)\end{array}$ & $118.85^{* * *}$ & 7208 \\
\hline M73 & $\begin{array}{c}.041 \\
(.02728)\end{array}$ & $\begin{array}{c}.83 \\
(.0238)\end{array}$ & $\begin{array}{c}1.175^{* * *} \\
(.05)\end{array}$ & $\begin{array}{c}-.02^{* * *} \\
(.00)\end{array}$ & $\begin{array}{c}-.048^{* * *} \\
(.01)\end{array}$ & $\begin{array}{c}.0027^{* * *} \\
(.00)\end{array}$ & $\begin{array}{c}.003^{* * *} \\
(.00)\end{array}$ & $647^{* * *}$ & 26002 \\
\hline M74 & $\begin{array}{c}.012 \\
(.00774)\end{array}$ & $\begin{array}{c}.831 \\
(.0129)\end{array}$ & $\begin{array}{c}.623^{* * *} \\
(.07)\end{array}$ & $\begin{array}{c}.015^{* * *} \\
(.00)\end{array}$ & $\begin{array}{c}.027^{* * *} \\
(.01)\end{array}$ & $\begin{array}{c}.0008^{* * *} \\
(.00)\end{array}$ & $\begin{array}{c}-.003^{* * *} \\
(.00)\end{array}$ & $342.96^{* * *}$ & 17242 \\
\hline M75 & $\begin{array}{c}.006 \\
(.00343)\end{array}$ & $\begin{array}{c}.814 \\
(.0684)\end{array}$ & $\begin{array}{c}2.417^{* * *} \\
(.16)\end{array}$ & $\begin{array}{c}-.114^{* * *} \\
(.01)\end{array}$ & $\begin{array}{c}-.072^{* * *} \\
(.01)\end{array}$ & $\begin{array}{c}-.0003 \\
(.00)\end{array}$ & $\begin{array}{c}.011 * * * \\
(.00)\end{array}$ & $41.3^{* * *}$ & 11214 \\
\hline N77 & $\begin{array}{c}.015 \\
(.01323)\end{array}$ & $\begin{array}{c}.877 \\
(.0183)\end{array}$ & $\begin{array}{c}.731^{* * *} \\
(.03)\end{array}$ & $\begin{array}{c}.012^{* * *} \\
(.00)\end{array}$ & $\begin{array}{c}.016^{* * * *} \\
(.00)\end{array}$ & $\begin{array}{c}.0012^{* * *} \\
(.00)\end{array}$ & $\begin{array}{c}-.002^{* * *} \\
(.00)\end{array}$ & $230.4^{* * *}$ & 18494 \\
\hline N78 & $\begin{array}{c}.049 \\
(.04287)\end{array}$ & $\begin{array}{c}.686 \\
(.0477)\end{array}$ & $\begin{array}{c}1.111^{* * * *} \\
(.09)\end{array}$ & $\begin{array}{c}-.016^{* * * *} \\
(.00)\end{array}$ & $\begin{array}{c}.044 * * * \\
(.01)\end{array}$ & $\begin{array}{c}.005^{* * *} \\
(.00)\end{array}$ & $\begin{array}{c}-.008^{* * *} \\
(.00)\end{array}$ & $520.46^{* * *}$ & 16880 \\
\hline N79 & $\begin{array}{c}.052 \\
(.02953)\end{array}$ & $\begin{array}{c}.782 \\
(.0684)\end{array}$ & $\begin{array}{c}1.73^{* * *} \\
(.09)\end{array}$ & $\begin{array}{c}-.045^{* * *} \\
(.00)\end{array}$ & $\begin{array}{r}-.007 \\
(.02)\end{array}$ & $\begin{array}{c}.0048^{* * *} \\
(.00)\end{array}$ & $\begin{array}{l}-.002 \\
(.00)\end{array}$ & $546.27^{* * *}$ & 14240 \\
\hline N80 & $\begin{array}{c}.104 \\
(.04347)\end{array}$ & $\begin{array}{l}.745 \\
(.0307)\end{array}$ & $\begin{array}{c}.349^{* * *} \\
(.1)\end{array}$ & $\begin{array}{c}.025^{* * *} \\
(.01)\end{array}$ & $\begin{array}{c}.098^{* * * *} \\
(.03)\end{array}$ & $\begin{array}{c}.009^{* * * *} \\
(.00)\end{array}$ & $\begin{array}{c}-.016^{* * *} \\
(.00)\end{array}$ & $296.34^{* * *}$ & 6746 \\
\hline N81 & $\begin{array}{c}.012 \\
(.02179)\end{array}$ & $\begin{array}{c}.843 \\
(.0057)\end{array}$ & $\begin{array}{c}.94^{* * *} \\
(.04)\end{array}$ & $\begin{array}{c}-.006^{* *} \\
(.00)\end{array}$ & $\begin{array}{c}-.016^{* * *} \\
(.00)\end{array}$ & $\begin{array}{c}.002^{* * *} \\
(.00)\end{array}$ & $\begin{array}{l}.00 \\
(.00)\end{array}$ & $774.99^{* * *}$ & 48348 \\
\hline N82 & $\begin{array}{c}.025 \\
(.01725)\end{array}$ & $\begin{array}{c}.875 \\
(.0277)\end{array}$ & $\begin{array}{c}.688^{* * *} \\
(.05)\end{array}$ & $\begin{array}{c}.014^{* * * *} \\
(.00)\end{array}$ & $\begin{array}{c}.058^{* * *} \\
(.01)\end{array}$ & $\begin{array}{c}.0023^{* * *} \\
(.00)\end{array}$ & $\begin{array}{c}-.008^{* * *} \\
(.00)\end{array}$ & $385.41^{* * *}$ & 22407 \\
\hline S95 & $\begin{array}{c}.01 \\
(.02187)\end{array}$ & $\begin{array}{c}.806 \\
(.0842)\end{array}$ & $\begin{array}{c}-.395^{* * *} \\
(.13)\end{array}$ & $\begin{array}{c}.08^{* * *} \\
(.01)\end{array}$ & $\begin{array}{c}.075^{* * *} \\
(.01)\end{array}$ & $\begin{array}{c}.0023^{* * *} \\
(.00)\end{array}$ & $\begin{array}{c}-.012^{* * *} \\
(.00)\end{array}$ & $122.76^{* * *}$ & 8586 \\
\hline
\end{tabular}

*** $\mathrm{p}<0.01,{ }^{* *} \mathrm{p}<0.05, * \mathrm{p}<0.1$. Standard errors in parentheses. Point estimates of the constant term unreported. $\mathrm{F}$ statistic for the test of joint significance of the $3 \mathrm{KBC}$ terms $\left(\beta_{c}, \beta_{c^{2}}\right.$ and $\left.\beta_{c \omega}\right)$ 
Figure 1: Marginal effect of KBC by quintiles of KBC Stock and industry
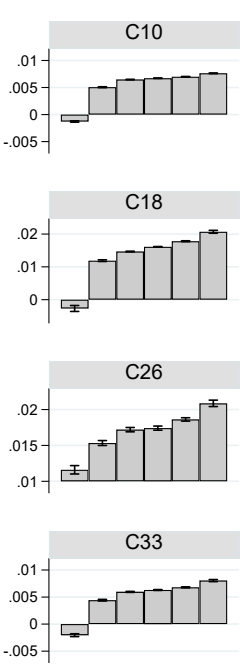
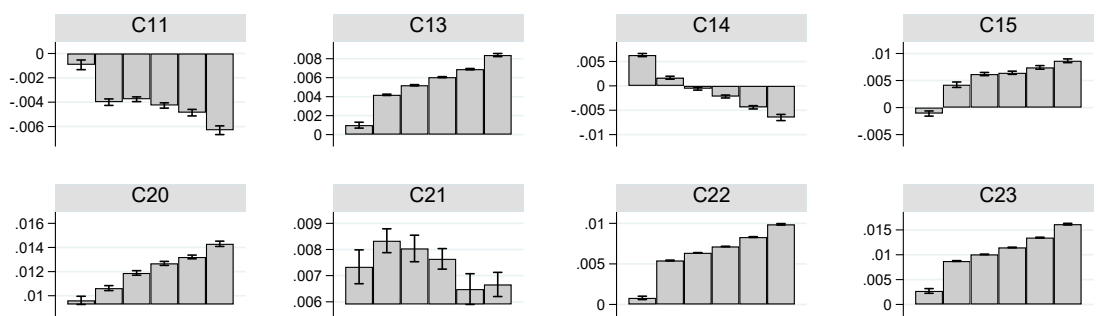

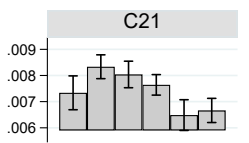
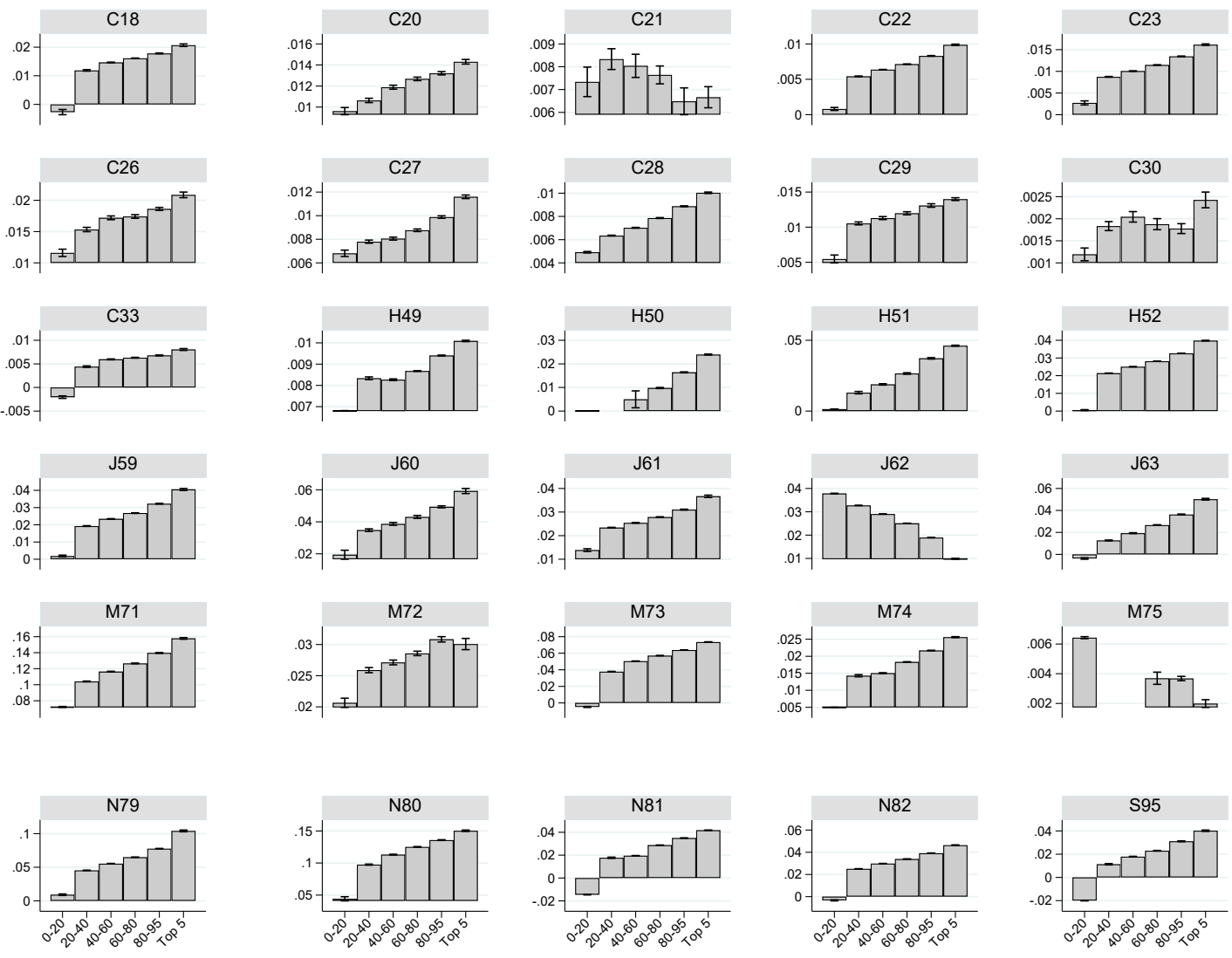

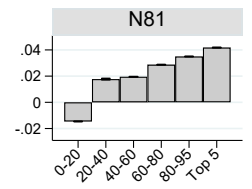

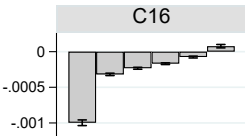

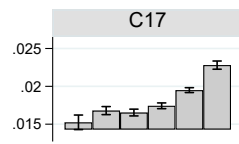
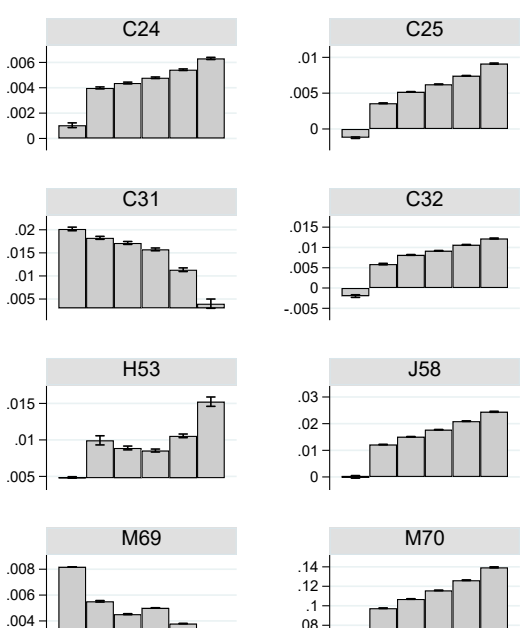

$.006-\left[\begin{array}{l}.006 \\ 0.7 \square\end{array}\right.$
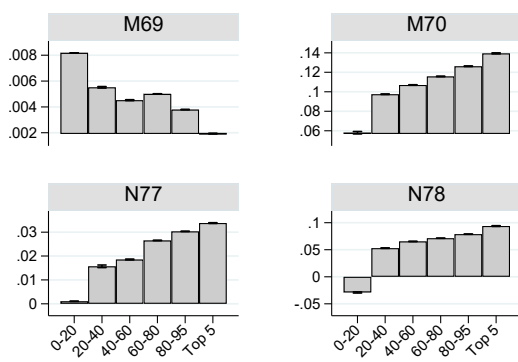

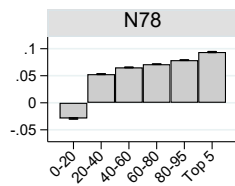

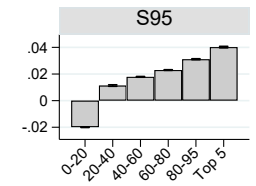

Quintiles of KBC stock 

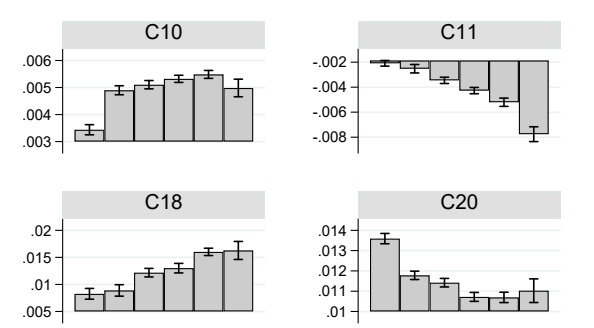

$\mathrm{C} 20$

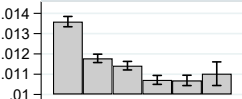

C26 $\left.\begin{array}{l}.018 \\ .016 \\ .015\end{array}\right] \mathrm{T}^{\mathrm{T}} \mathrm{I}_{\mathrm{I}_{\mathrm{I}_{1} \mathrm{I}}}$

$\left.\begin{array}{c}\text { C33 } \\ .006-1 \\ .005-1 \\ .003\end{array}\right]\left.\mathrm{I}^{\mathrm{I}} \mathrm{I}^{\mathrm{I}} \mathrm{I}^{\mathrm{I}}\right|^{\mathrm{I}}$

$\mathrm{C} 27$
$.009-\mathrm{I}_{.007}$

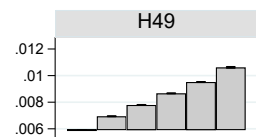

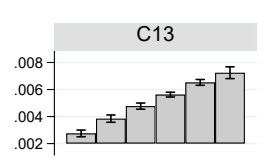

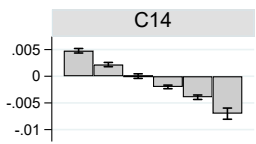

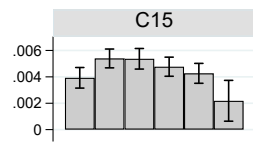

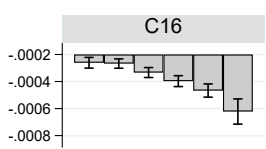
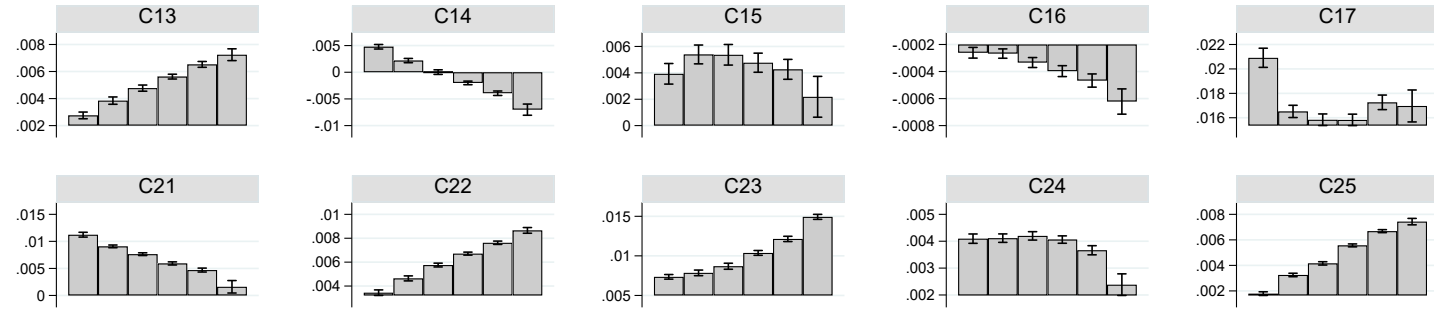

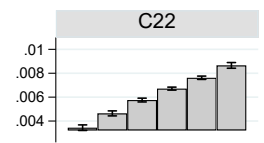

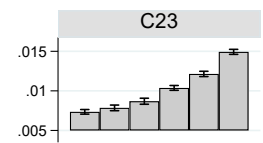

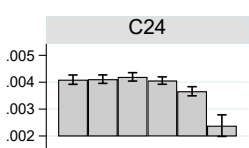
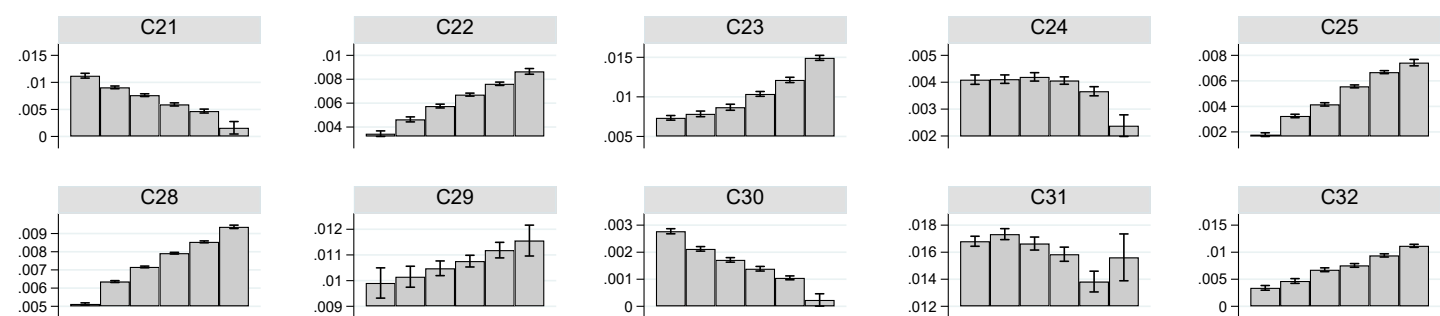

\begin{tabular}{c} 
C29 \\
$.012-11$ \\
.01 \\
$.009-1-T^{-}-\left.\left.{ }^{I}\right|^{I}\right|^{I}-$ \\
\hline
\end{tabular}

$\left.\begin{array}{c}\text { C30 } \\ .003 \\ .002 \\ 0\end{array}\right]\left.\right|^{x}=$

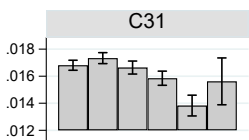

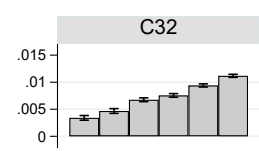

$.008-$
.006
.006
.002 $\mathrm{I}^{\mathrm{I}} \mathrm{I}^{\mathrm{I}} \mathrm{I}^{\mathrm{I}} \mathrm{I}$
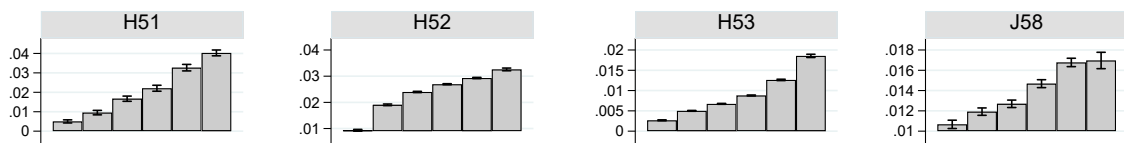

J59

$\mathrm{J} 60$

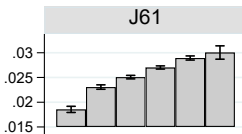

J62

$\begin{aligned} & .032-1 \\ & .03-1 \\ & 028\end{aligned}-$

.

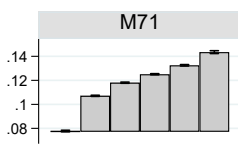
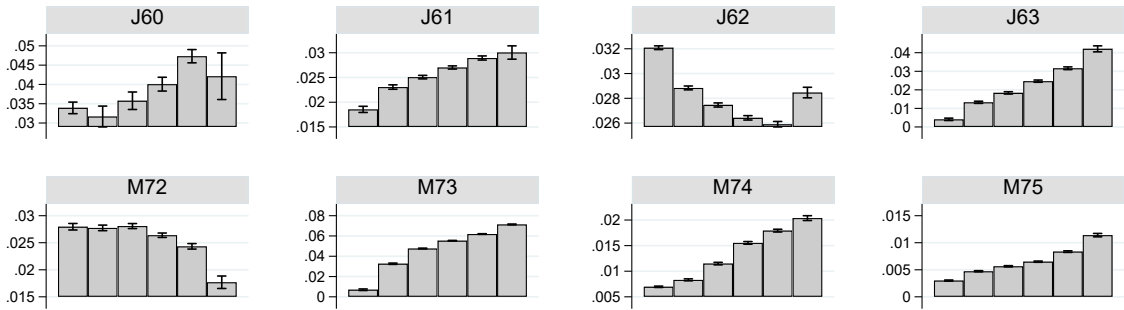

$\left.\begin{array}{c}\text { M69 } \\ .0075-1.007 \\ .0065 \\ .006\end{array}\right]$

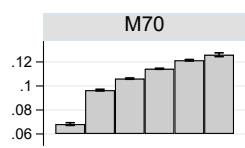

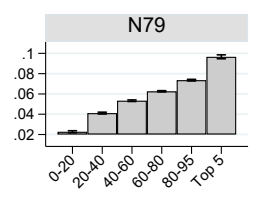
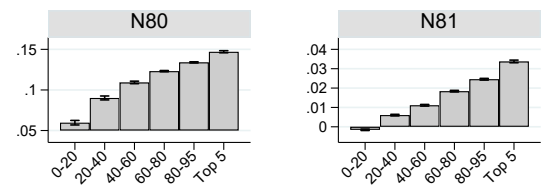
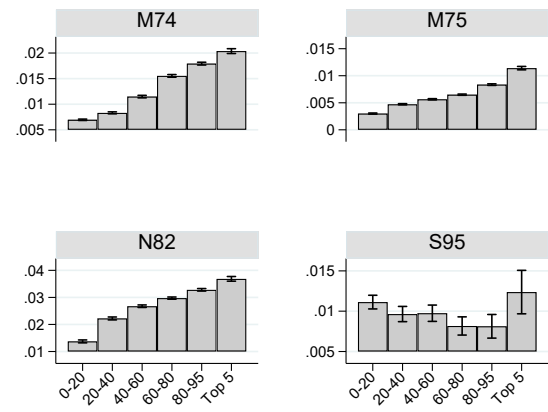

.015

Quintiles of productivity

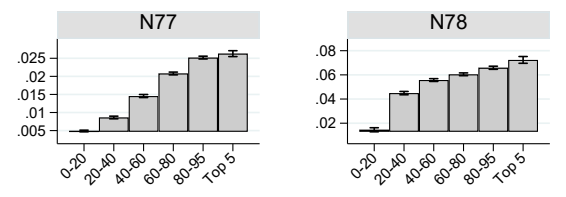

Marginal effect of KBC

+2 std. err/- 2 std. err 
The evidence supports the presence of increasing returns to the size of KBC stock, as the coefficient $\beta_{c^{2}}$ is significantly positive in all but 6 of the 47 industries. Figure 1 offers a visualisation of the increasing returns to KBC stock, by plotting the average $\theta_{c}$ by quintile of $\mathrm{KBC}$ stock, with an additional group for the top $5 \%$, which represent the $\mathrm{KBC}$ frontier. First, we observe that the elasticity of $\mathrm{KBC}$ is negative in the bottom quintile in 14 of the 47 industries, implying that there is a minimum scale for KBC to be effective. Second, the effect of $\mathrm{KBC}$ on productivity increases across the distribution of KBC stock. Third, we find that the elasticity of KBC on the frontier is sizeably larger than the average $\theta_{c}$. The exceptions to these patterns are the six sectors with a negative $\beta_{c^{2}}$, and hence which not display increasing returns to KBC.

Across all industries analysed, $\theta_{c}$ is on average 3 times higher in the top quintile of the KBC distribution compared to the bottom quintile. Moreover, in the manufacturing sector, $\theta_{c}$ is at least $22 \%$ larger for frontier firms than for the average firm in the chemical industry (C20), and twice as large in metal products (C25). In the services sector, the effect of $\mathrm{KBC}$ for frontier firms is at least double that for the average firm in nine industries $^{22}$, reaching four times larger in water transport (H50). This evidence strongly supports the presence of increasing returns in the size of KBC stock, and the possibility of KBC driving productivity divergence between top KBC users and the rest.

Finally, we do not find evidence that firms which are in a better position on the productivity distribution have an advantage to reap the benefits of $\mathrm{KBC}$ stocks. Indeed, the coefficient $\beta_{c \omega}$ is negative in 34 of the 47 industries, and the distribution of $\theta_{c}$ across quintiles of productivity, shown in Figure 2 is much flatter than across quintiles of KBC stock. This further strengthens our interpretation that it is the overall size of firm's KBC stock that yields increasing returns.

\subsection{Robustness estimations of the law of motion of productivity}

In the present subsection, we test the sensitivity of our estimation of the law of motion of productivity with respect to three assumptions. We present the main coefficients of interest in Tables 5 and 6 , and report the full estimation results in Appendix C.

First, we relax the functional form assumption of $g(\cdot)$, by estimating a linear specification of the form

$$
\omega_{i t}=\beta_{\omega} \omega_{i t-1}+\beta_{c} c_{i t-1}+u_{i t}
$$

The second column of Tables 5 and 6 reports the point estimate for $\beta_{c}$, which is the estimate for the average elasticity of KBC on productivity, and hence can be directly compared to the $\theta_{c}$ reported in Tables 3 and 4 . Across all industries, the positive effect of $\mathrm{KBC}$ is confirmed, although $\beta_{c}$ is generally of a smaller magnitude than average $\theta_{c}$. Estimation results in some industries in the business service and the administrative services

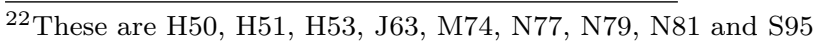


sector are sensitive to the functional form assumption. This is especially the case for consulting services (M70), architectural and engineering design (M71), and security and investigation activities (N80), where a large drop between the average $\theta_{c}$ and $\beta_{c}$ can be observed. Note, those are also the industries with the high $\theta_{c}$ in the main specification. However, there are virtually no differences in $\theta_{c}$ in 5 of the 12 sub-sectors of the business service and the administrative services sector.

Second, we test the sensitivity of our results to the constructed measure of organisational capital, by using only the sum of software, IPP and R\&D as the measure of KBC. The second and third columns of Tables 5 and 6 report the average $\theta_{c, n o O C}$, the elasticity of $\mathrm{KBC}$ excluding organisational capital, and the respective F-statistic. The results confirm the positive and significant impact of $\mathrm{KBC}$ on productivity established in Section 5.1, and provide reassurance that this positive association is not artificially driven by our measure of organisational capital. Nevertheless, the elasticity of KBC when organisational capital is excluded is lower in magnitude in most industries. There are two potential explanations for this finding. On the one hand, organisational capital has been shown to have a positive effect on firm performance (Bloom et al., 2013; Crass and Peters, 2014), and hence its omission will lead to a lower estimated effect of total KBC stock. On the other hand, the measure of organisational capital contains measurement error and part of the additional cross-industry variation in the $\mathrm{KBC}$ coefficient can thus be attributed to the larger noise in the variable. As the magnitude of $\theta_{c, n o O C}$ is only slightly smaller in most industries, we interpret these differences mainly to be due to the first explanation.

Overall, we find that the results are sensitive to the inclusion of organisational capital in one manufacturing industry and four service sectors. In the former, the beverage industry (C11), which was the only industry with a negative output elasticity of $\mathrm{KBC}$ in the main specification, has a positive elasticity when organisational capital is excluded, further reinforcing the result that $\mathrm{KBC}$ improves productivity. However, in services, three sectors seem to be sensitive to the inclusion of organisational capital, as the elasticity is noticeably lower without this asset. These are temporary employment agencies, travel agencies, and security and investigation activities (N78, N79 and N80), which are partly also sensitive to the functional form assumption of the law of motion.

Finally, we re-estimate our model with KBC investment flows as opposed to stocks. Our main specification follows Corrado et al. (2005, 2009), by capitalising KBC expenditure to account for the fact the effect of KBC is cumulative and affects firm performance over multiple years. Corrado et al. (2005, 2009) argue that KBC should be treated analogously to tangible capital. However, as expenditures or invesment flows of KBC are often used in the literature, we also report results using this latter measure. Columns five and six of Tables 5 and 6 display the average $\theta_{c, \text { Invest }}$, which uses KBC investment instead of stocks, and the respective F-statistic. The average marginal effect is positive and significant in most industries, but also of smaller magnitude. This is in line with our assumption that KBC has a beneficial effect over multiple years and supports the results of the main specification. The industries which seem most sensitive to this measure are the same ones flagged by the other robustness checks. 
Table 5: Results of robustness estimations, by 2-digit industry in Manufacturing

\begin{tabular}{|c|c|c|c|c|c|c|}
\hline \multirow{2}{*}{$\begin{array}{r}\text { Functional form: } \\
\text { Assets: } \\
\text { Industry }\end{array}$} & \multirow{2}{*}{$\begin{array}{c}\text { Linear } \\
\text { All elements } \\
\text { Stock } \\
\beta_{c}\end{array}$} & \multicolumn{2}{|c|}{$\begin{array}{c}\text { Translog } \\
\text { No OC } \\
\text { Stock }\end{array}$} & \multicolumn{2}{|c|}{$\begin{array}{c}\text { Translog } \\
\text { All elements } \\
\text { Investment }\end{array}$} & \multirow[b]{2}{*}{$\mathrm{N}$} \\
\hline & & $\theta_{c, n o O C}$ & $\mathrm{~F}$ & $\theta_{c, \text { Invest }}$ & $\mathrm{F}$ & \\
\hline $\mathrm{C} 10$ & $\begin{array}{c}.002^{* * *} \\
(.00)\end{array}$ & $\begin{array}{c}.002 \\
(.00268)\end{array}$ & $22.65^{* * *}$ & $\begin{array}{c}.001 \\
(.0017)\end{array}$ & $25.08^{* * *}$ & 9482 \\
\hline $\mathrm{C} 11$ & $\begin{array}{c}.00 \\
(.00)\end{array}$ & $\begin{array}{c}.002 \\
(.00143)\end{array}$ & $2.92 * *$ & $\begin{array}{c}.003 \\
(.00449)\end{array}$ & $2.65^{* *}$ & 1297 \\
\hline $\mathrm{C} 13$ & $\begin{array}{l}.001 \\
(.00)\end{array}$ & $\begin{array}{c}0 \\
(.00135)\end{array}$ & 2.05 & $\begin{array}{c}.008 \\
(.00739)\end{array}$ & $6.53^{* * *}$ & 1929 \\
\hline $\mathrm{C} 14$ & $\begin{array}{c}.003^{* * *} \\
(.00)\end{array}$ & $\begin{array}{c}.001 \\
(.0045)\end{array}$ & $4.07^{* * *}$ & $\begin{array}{c}0 \\
(.00576)\end{array}$ & $4.84^{* * *}$ & 1053 \\
\hline $\mathrm{C} 15$ & $\begin{array}{l}.002 \\
(.00)\end{array}$ & $\begin{array}{c}.001 \\
(.00305)\end{array}$ & .92 & $\begin{array}{c}.005 \\
(.01083)\end{array}$ & $2.96^{* *}$ & 469 \\
\hline $\mathrm{C} 16$ & $\begin{array}{r}-.001 \\
(.00)\end{array}$ & $\begin{array}{c}-.001 \\
(.00161)\end{array}$ & 1.12 & $\begin{array}{c}-.002 \\
(.00382)\end{array}$ & 1.47 & 1994 \\
\hline $\mathrm{C} 17$ & $\begin{array}{c}.011^{* * *} \\
(.00)\end{array}$ & $\begin{array}{c}.002 \\
(.00143)\end{array}$ & $7.32^{* * *}$ & $\begin{array}{c}.01 \\
(.00322)\end{array}$ & $16.26^{* * *}$ & 2257 \\
\hline $\mathrm{C} 18$ & $\begin{array}{c}.002^{* * *} \\
(.00)\end{array}$ & $\begin{array}{c}.004 \\
(.00619)\end{array}$ & $13.35^{* * *}$ & $\begin{array}{c}.013 \\
(.01735)\end{array}$ & $18.66^{* * *}$ & 1635 \\
\hline $\mathrm{C} 20$ & $\begin{array}{c}.007^{* * *} \\
(.00)\end{array}$ & $\begin{array}{c}.004 \\
(.00232)\end{array}$ & $16.09 * * *$ & $\begin{array}{c}.009 \\
(.00236)\end{array}$ & $33.29 * * *$ & 4222 \\
\hline $\mathrm{C} 21$ & $\begin{array}{c}.008^{* * *} \\
(.00)\end{array}$ & $\begin{array}{c}.004 \\
(.0032)\end{array}$ & $3.77^{* *}$ & $\begin{array}{l}-.001 \\
(.002)\end{array}$ & 1.48 & 913 \\
\hline $\mathrm{C} 22$ & $\begin{array}{c}.002^{* * *} \\
(.00)\end{array}$ & $\begin{array}{c}.002 \\
(.00199)\end{array}$ & $9.98^{* * *}$ & $\begin{array}{c}.003 \\
(.00226)\end{array}$ & $10.84^{* * *}$ & 4573 \\
\hline $\mathrm{C} 23$ & $\begin{array}{l}.001^{*} \\
(.00)\end{array}$ & $\begin{array}{c}.003 \\
(.00336)\end{array}$ & $13.22^{* * *}$ & $\begin{array}{c}.003 \\
(.00079)\end{array}$ & $8.7 * * *$ & 3763 \\
\hline $\mathrm{C} 24$ & $\begin{array}{c}.001^{* * *} * \\
(.00)\end{array}$ & $\begin{array}{c}.001 \\
(.00045)\end{array}$ & $3.88^{* * *}$ & $\begin{array}{c}0 \\
(.00108)\end{array}$ & .8 & 3275 \\
\hline $\mathrm{C} 25$ & $\begin{array}{c}.001^{* * *} \\
(.00)\end{array}$ & $\begin{array}{c}.001 \\
(.00132)\end{array}$ & $15.37^{* * *}$ & $\begin{array}{c}.002 \\
(.00207)\end{array}$ & $28.12^{* * *}$ & 10430 \\
\hline $\mathrm{C} 26$ & $\begin{array}{c}.009^{* * *} \\
(.00)\end{array}$ & $\begin{array}{c}.008 \\
(.00421)\end{array}$ & $32.33^{* * *}$ & $\begin{array}{c}.011 \\
(.00643)\end{array}$ & $26.34^{* * *}$ & 3664 \\
\hline $\mathrm{C} 27$ & $\begin{array}{c}.005^{* * *} \\
(.00)\end{array}$ & $\begin{array}{c}.004 \\
(.00229)\end{array}$ & $27.27^{* * *}$ & $\begin{array}{c}.002 \\
(.00152)\end{array}$ & $8.51^{* * *}$ & 5063 \\
\hline $\mathrm{C} 28$ & $\begin{array}{c}.005^{* * *} \\
(.00)\end{array}$ & $\begin{array}{c}.004 \\
(.00172)\end{array}$ & $61.91^{* * *}$ & $\begin{array}{c}.002 \\
(.00076)\end{array}$ & $15.18^{* * *}$ & 11500 \\
\hline $\mathrm{C} 29$ & $\begin{array}{c}.005^{* * *} \\
(.00)\end{array}$ & $\begin{array}{c}.005 \\
(.00503)\end{array}$ & $20 * * *$ & $\begin{array}{c}.007 \\
(.00409)\end{array}$ & $16.92^{* * *}$ & 2974 \\
\hline C30 & $\begin{array}{l}.001 \\
(.00)\end{array}$ & $\begin{array}{c}.001 \\
(.00098)\end{array}$ & .31 & $\begin{array}{c}0 \\
(.00068)\end{array}$ & .34 & 1000 \\
\hline C31 & $\begin{array}{c}.015^{* * * *} \\
(.00)\end{array}$ & $\begin{array}{c}.002 \\
(.002)\end{array}$ & $3.56^{* *}$ & $\begin{array}{c}.004 \\
(.00655)\end{array}$ & $12.51^{* * *}$ & 1875 \\
\hline C32 & $\begin{array}{c}.001^{* * *} \\
(.00)\end{array}$ & $\begin{array}{c}.003 \\
(.00302)\end{array}$ & $8.63^{* * *}$ & $\begin{array}{c}.004 \\
(.00679)\end{array}$ & $12.18^{* * *}$ & 3042 \\
\hline C33 & $\begin{array}{c}.001^{* *} \\
(.00)\end{array}$ & $\begin{array}{c}.001 \\
(.00093)\end{array}$ & $4.55^{* * *}$ & $\begin{array}{c}.004 \\
(.00607)\end{array}$ & $11.83^{* * *}$ & 3171 \\
\hline
\end{tabular}

${ }^{* * *} \mathrm{p}<0.01,{ }^{*} * \mathrm{p}<0.05,{ }^{*} \mathrm{p}<0.1$. Standard errors in parentheses. 
Table 6: Results of robustness estimations, by 2-digit industry in Services

\begin{tabular}{|c|c|c|c|c|c|c|}
\hline \multirow{2}{*}{$\begin{array}{r}\text { Functional form: } \\
\text { Assets: } \\
\text { Industry }\end{array}$} & \multirow{2}{*}{$\begin{array}{c}\text { Linear } \\
\text { All elements } \\
\text { Stock } \\
\beta_{c} \\
\end{array}$} & \multicolumn{2}{|c|}{$\begin{array}{c}\text { Translog } \\
\text { No OC } \\
\text { Stock }\end{array}$} & \multicolumn{2}{|c|}{$\begin{array}{c}\text { Translog } \\
\text { All elements } \\
\text { Investment }\end{array}$} & \multirow[b]{2}{*}{$\mathrm{N}$} \\
\hline & & $\theta_{c, n o O C}$ & F & $\theta_{c, \text { Invest }}$ & $\mathrm{F}$ & \\
\hline H49 & $\begin{array}{c}.008^{* * *} \\
(.00)\end{array}$ & $\begin{array}{c}.007 \\
(.00278)\end{array}$ & $225.26 * * *$ & $\begin{array}{c}.005 \\
(.00282)\end{array}$ & $402.99^{* * *}$ & 75957 \\
\hline H50 & $\begin{array}{c}.009^{* * *} \\
(.00)\end{array}$ & $\begin{array}{c}.005 \\
(.00686)\end{array}$ & $29.47^{* * *}$ & $\begin{array}{c}.012 \\
(.00179)\end{array}$ & $30.45^{* * *}$ & 7454 \\
\hline H51 & $\begin{array}{c}.023^{* * *} \\
(.00)\end{array}$ & $\begin{array}{c}.018 \\
(.01073)\end{array}$ & $39.7^{* * *}$ & $\begin{array}{c}.009 \\
(.00241)\end{array}$ & $6.88^{* * *}$ & 1482 \\
\hline H52 & $\begin{array}{c}.012^{* * *} \\
(.00)\end{array}$ & $\begin{array}{c}.008 \\
(.00225)\end{array}$ & $281.12^{* * *}$ & $\begin{array}{c}.01 \\
(.00136)\end{array}$ & $372.77^{* * *}$ & 32179 \\
\hline H53 & $\begin{array}{c}.007^{* * *} \\
(.00)\end{array}$ & $\begin{array}{c}.01 \\
(.00157)\end{array}$ & $57.15^{* * *}$ & $\begin{array}{c}.003 \\
(.00398)\end{array}$ & $25.1^{* * *}$ & 7820 \\
\hline J58 & $\begin{array}{c}.003^{* * *} \\
(.00)\end{array}$ & $\begin{array}{c}.002 \\
(.00728)\end{array}$ & $20.96^{* * *}$ & $\begin{array}{c}-.001 \\
(.00844)\end{array}$ & $57.46^{* * *}$ & 10931 \\
\hline J59 & $\begin{array}{c}.012^{* * *} \\
(.00)\end{array}$ & $\begin{array}{c}.006 \\
(.0018)\end{array}$ & $21.52^{* * *}$ & $\begin{array}{c}.001 \\
(.02092)\end{array}$ & $45.62^{* * *}$ & 5869 \\
\hline $\mathrm{J} 60$ & $\begin{array}{c}.02^{* * *} \\
(.00)\end{array}$ & $\begin{array}{c}.013 \\
(.01412)\end{array}$ & $4.17^{* * *}$ & $\begin{array}{c}.012 \\
(.00557)\end{array}$ & $6.78^{* * *}$ & 838 \\
\hline J61 & $\begin{array}{c}.015^{* * *} \\
(.00)\end{array}$ & $\begin{array}{c}.006 \\
(.00568)\end{array}$ & $11.28^{* * *}$ & $\begin{array}{c}.01 \\
(.00426)\end{array}$ & $19.52^{* * *}$ & 4212 \\
\hline $\mathrm{J} 62$ & $\begin{array}{c}.028^{* * *} \\
(.00)\end{array}$ & $\begin{array}{c}0 \\
(.00102)\end{array}$ & 1.57 & $\begin{array}{c}.008 \\
(.01319)\end{array}$ & $280.5^{* * *}$ & 43097 \\
\hline $\mathrm{J} 63$ & $\begin{array}{c}.005^{* * *} \\
(.00)\end{array}$ & $\begin{array}{c}.007 \\
(.01253)\end{array}$ & $49.57^{* * *}$ & $\begin{array}{c}.006 \\
(.0167)\end{array}$ & $82.5^{* * *}$ & 10904 \\
\hline M69 & $\begin{array}{c}.006^{* * *} \\
(.00)\end{array}$ & $\begin{array}{c}.007 \\
(.00288)\end{array}$ & $417.88^{* * *}$ & $\begin{array}{c}-.009 \\
(.00688)\end{array}$ & $97.82^{* * *}$ & 81249 \\
\hline M70 & $\begin{array}{c}.027^{* * *} \\
(.00)\end{array}$ & $\begin{array}{c}.102 \\
(.0436)\end{array}$ & $1523.31^{* * *}$ & $\begin{array}{c}.031 \\
(.01545)\end{array}$ & $431.22 * * *$ & 32538 \\
\hline M71 & $\begin{array}{c}.076^{* * *} \\
(.00)\end{array}$ & $\begin{array}{c}.131 \\
(.04754)\end{array}$ & $4220.81^{* * *}$ & $\begin{array}{c}.071 \\
(.03662)\end{array}$ & $26422.42^{* * *}$ & 59139 \\
\hline M72 & $\begin{array}{c}.017^{* * *} \\
(.00)\end{array}$ & $\begin{array}{c}.002 \\
(.00314)\end{array}$ & $14.64^{* * *}$ & $\begin{array}{c}.019 \\
(.01364)\end{array}$ & $60.24^{* * *}$ & 7208 \\
\hline M73 & $\begin{array}{c}.014^{* * *} \\
(.00)\end{array}$ & $\begin{array}{c}.031 \\
(.02563)\end{array}$ & $519.57^{* * *}$ & $\begin{array}{c}.022 \\
(.01638)\end{array}$ & $292.38^{* * *}$ & 26002 \\
\hline M74 & $\begin{array}{c}.012^{* * *} \\
(.00)\end{array}$ & $\begin{array}{c}.011 \\
(.00262)\end{array}$ & $253.35^{* * *}$ & $\begin{array}{c}-.001 \\
(.0058)\end{array}$ & $16.82^{* * *}$ & 17242 \\
\hline M75 & $\begin{array}{c}.006^{* * *} \\
(.00)\end{array}$ & $\begin{array}{c}.006 \\
(.0035)\end{array}$ & $40.9^{* * *}$ & $\begin{array}{c}-.019 \\
(.00826)\end{array}$ & $15.2^{* * *}$ & 11214 \\
\hline N77 & $\begin{array}{c}.014^{* * *} \\
(.00)\end{array}$ & $\begin{array}{c}.014 \\
(.01266)\end{array}$ & $212.88^{* * *}$ & $\begin{array}{c}.003 \\
(.02022)\end{array}$ & $97.6^{* * *}$ & 18494 \\
\hline N78 & $\begin{array}{c}.01^{* * *} \\
(.00)\end{array}$ & $\begin{array}{c}.003 \\
(.00881)\end{array}$ & $46.42^{* * *}$ & $\begin{array}{c}.015 \\
(.0219)\end{array}$ & $204.01^{* * *}$ & 16880 \\
\hline N79 & $\begin{array}{c}.021^{* * *} \\
(.00)\end{array}$ & $\begin{array}{c}.01 \\
(.00281)\end{array}$ & $162.82^{* * *}$ & $\begin{array}{c}.016 \\
(.00749)\end{array}$ & $395.12^{* * *}$ & 14240 \\
\hline N80 & $\begin{array}{c}.019 * * * \\
(.00)\end{array}$ & $\begin{array}{c}.005 \\
(.00114)\end{array}$ & $22.37^{* * *}$ & $\begin{array}{c}.014 \\
(.01706)\end{array}$ & $52.89^{* * *}$ & 6746 \\
\hline N81 & $\begin{array}{c}.005^{* * *} \\
(.00)\end{array}$ & $\begin{array}{c}.004 \\
(.00226)\end{array}$ & $139.97^{* * *}$ & $\begin{array}{c}.002 \\
(.01393)\end{array}$ & $317.62^{* * *}$ & 48348 \\
\hline N82 & $\begin{array}{c}.01^{* * *} \\
(.00)\end{array}$ & $\begin{array}{c}.008 \\
(.00254)\end{array}$ & $169.35^{* * *}$ & $\begin{array}{c}.007 \\
(.00181)\end{array}$ & $132.07^{* * *}$ & 22407 \\
\hline S95 & $\begin{array}{c}.003^{* * *} \\
(.00)\end{array}$ & $\begin{array}{c}.008 \\
(.0037)\end{array}$ & 25 & $\begin{array}{c}.005 \\
(.01298)\end{array}$ & $65.22^{* * *}$ & 8586 \\
\hline
\end{tabular}




\section{Productivity dynamics at the industry level}

\subsection{Aggregate productivity dynamics}

Hereafter, we test our second hypothesis and present evidence illustrating the link between KBC and productivity divergence. We adopt the approach of Andrews et al. (2019) and compare the productivity performance of two groups of firms: a group of top performers, which we call "frontier" firms, and a representative group of all other firms, which we call "followers". We define the group of frontier firms according to two criteria and compare their performance. On the one hand, we replicate the methodology of Andrews et al. (2019) and define the TFP frontier as the top 5\% most productive firms in each industry and year. On the other hand, we define the $\mathrm{KBC}$ frontier as the group of frontier firms with the top $5 \%$ largest KBC stocks in each industry and year. For both methodologies, we allow the identity of firms that populate the frontier group to differ across years. Our results should therefore be interpreted as representing changes in the upper tail of the distributions, of TFP or KBC stock, rather than the behaviour of a representative firm in the respective group. The results based on the TFP frontier are directly comparable to those of Andrews et al. (2019) and will therefore be used to verify whether the divergence results found in the global context also hold in Germany. The results based on the KBC frontier extend this discussion to analyse whether $\mathrm{KBC}$ has a role to play in divergence.

Figures 3 and 4 depict the evolution of productivity, relative to the base year, in firms on the TFP frontier (dotted line), firms on the KBC frontier (dashed line) and firms in the rest (solid), for Manufacturing and Services respectively. ${ }^{23}$ In both aggregate sectors, we find that the evolution of productivity on the TFP frontier closely follows the evolution of productivity of the industry average. This suggests that there is no evidence of divergence between frontier and follower firms, when the frontier is defined along the TFP dimension. This lack of divergence in Germany is also documented in Schiersch (2019), who looks at different measures productivity, such as labour productivity. This is also in line with Bersch et al. (2019), who look at the evolution of the interquartile range of labour productivity in five German manufacturing industries. They find heterogeneous results across the six different industries they analyse ${ }^{24}$, and argue that this might be due to the different intensity with which the industries make use of KBC.

In contrast, these figures do indicate the presence of divergence along the KBC dimension. In the Manufacturing sector, while the productivity of the average firm grew by $6 \%$ over the period 2009 to 2014 , productivity on the KBC frontier grew by $9 \%$, resulting in a cumulative productivity gap of 3 percentage points over 5 years. In the service sector, the difference in growth between 2004 and 2013 is 9.5 percentage points. This supports

${ }^{23}$ Due to the change in industry classification system between 2007 and 2008, the statistical office significantly changed the design and sample size of the service survey. In order to overcome this break in the aggregated time series, 2003 and 2008 are used as base years in Figure 4. The growth between 2007 and 2008 is interpolated.

${ }^{24}$ The authors find that productivity divergence is increasing in the chemical, electrical engineering and mechanical engineering sectors, constant in the motor vehicle sector, and decreasing in optics, measurement and control technology, and medical equipment sectors. 
our hypothesis that $\mathrm{KBC}$ is a likely driver of productivity divergence.

Figure 3: Evolution of Productivity in Manufacturing

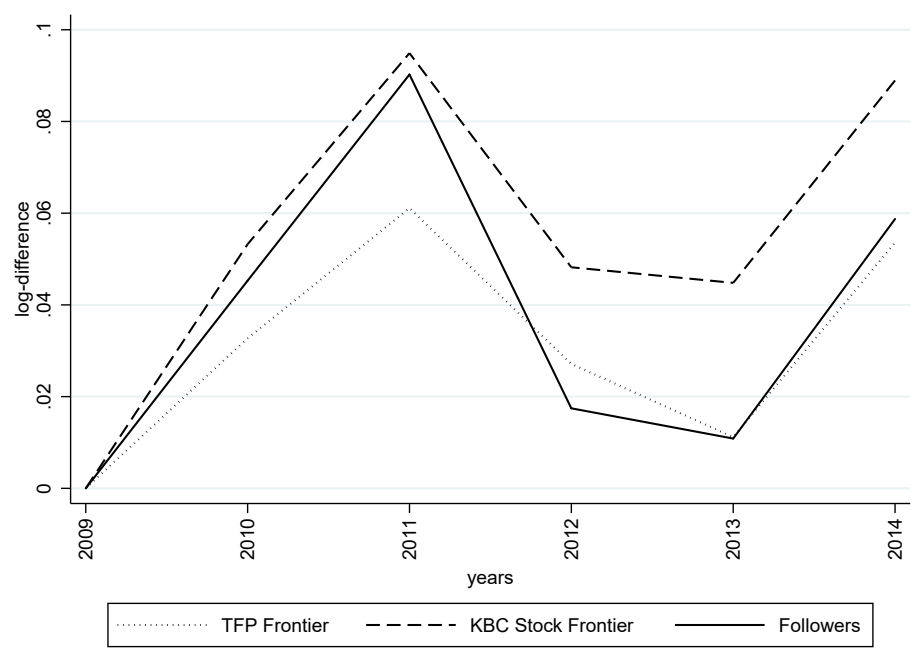

Figure 4: Evolution of Productivity in Services

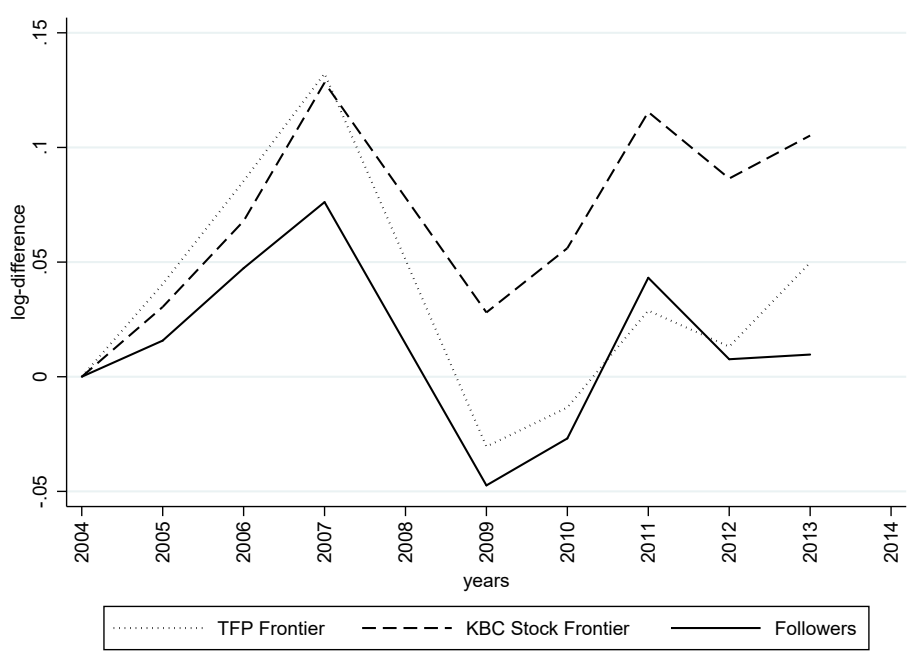


Table 7: Characteristics of Frontier and Follower firms

\begin{tabular}{|c|c|c|c|c|c|c|}
\hline \multirow[b]{3}{*}{ Variable } & \multicolumn{6}{|c|}{ Manufacturing } \\
\hline & \multicolumn{3}{|c|}{ Frontier defined by KBC } & \multicolumn{3}{|c|}{ Frontier defined by TFP } \\
\hline & Followers & Frontier & Sign.Diff. & Followers & Frontier & Sign.Diff. \\
\hline TFP & 7.98 & 8.47 & $* * *$ & 7.97 & 8.7 & $* * *$ \\
\hline Value Added & 9.73 & 218.93 & $* * *$ & 13.28 & 152.29 & $* * *$ \\
\hline Employment $^{\dagger}$ & 152.42 & 2312.41 & $* * *$ & 197.07 & 1475.02 & $* * *$ \\
\hline Capital & 20.01 & 414.86 & $* * *$ & 27.7 & 270.67 & $* * *$ \\
\hline Wages & 32.76 & 48.1 & $* * *$ & 32.41 & 54.61 & $* * *$ \\
\hline KBC Stock & 1.81 & 234.81 & $* * *$ & 5.89 & 158.42 & $* * *$ \\
\hline KBC Intensity ${ }^{\ddagger}$ & .09 & .48 & $* * *$ & .09 & .4 & $* * *$ \\
\hline Labor Productivity & 10.8 & 11.25 & $* * *$ & 10.79 & 11.4 & $* * *$ \\
\hline \multirow[t]{2}{*}{$\mathrm{N}$} & 102173 & 5448 & $* * *$ & 102173 & 5448 & $* * *$ \\
\hline & \multicolumn{6}{|c|}{ Service } \\
\hline Variable & Followers & Frontier & Sign.Diff. & Followers & Frontier & Sign.Diff. \\
\hline TFP & 8.09 & 9.01 & $* * *$ & 8.06 & 9.48 & $* * *$ \\
\hline Value Added & 1.25 & 38.05 & $* * *$ & 1.74 & 28.84 & $* * *$ \\
\hline Employment $^{\dagger}$ & 27.69 & 578.47 & $* * *$ & 38.79 & 368.15 & $* * *$ \\
\hline Capital & 2.97 & 124.01 & $* * *$ & 4.53 & 94.44 & $* * *$ \\
\hline Wages & 23.01 & 42.7 & $* * *$ & 21.37 & 73.79 & $* * *$ \\
\hline KBC Stock & .29 & 19.92 & $* * *$ & .61 & 13.98 & $* * *$ \\
\hline KBC Intensity $\ddagger$ & .94 & 2.81 & $* * *$ & .85 & 4.63 & $* * *$ \\
\hline Labor Productivity & 10.55 & 10.92 & $* * *$ & 10.53 & 11.38 & $* * *$ \\
\hline $\mathrm{N}$ & 801209 & 42295 & $* * *$ & 801209 & 42295 & $* * *$ \\
\hline
\end{tabular}

*** $\mathrm{p}<0.01$. Monetary values in million $€{ }^{\dagger}:$ head-count number of employees, ${ }^{\ddagger}$ percent.

Table 7 shows the descriptive statistics of the main variables of interest of frontier firms and followers, according to both definitions of the frontier. Frontier firms, irrespective of the definition of the frontier, show a significant size advantage, which is reminiscent of the findings of Andrews et al. (2019). Indeed, frontier firms are significantly larger in terms of value-added, employment, and capital, and pay significantly higher wages. The size difference is even more marked in services than in manufacturing. For example, average employment of firms on the TFP frontier in manufacturing is 7.5 times larger than average employment of follower firms. In services, the firms on the TFP frontier have 9.5 times more workers than followers.

In addition to this size difference, the level of TFP is also higher on both frontiers. Along the TFP frontier, the level difference holds by definition. Even though Figures 3 and 4 do not show evidence for divergence in TFP growth along the TFP frontier, there is still divergence in TFP levels. In the respective base years, TFP level on the frontier was twice the magnitude in the manufacturing sector (base year 2009) and in the services sector, it was 4 times larger on the frontier (base year 2004). Given the similar growth rates of TFP in both frontier and follower groups, divergence in the level of TFP has therefore increased, a result in line with Andrews et al. (2019).

Finally, these descriptive results provide further support for the "increased role for intangible capital in production" (Andrews et al., 2019, pp.4) as a source of divergence. Along the KBC frontier, the KBC stocks 
differ by a factor of 130 in manufacturing and 70 in services, while along the TFP frontier, this difference is only a factor 30 in manufacturing and 23 in services.

Table 8 shows the degree of persistence on the frontier, by reporting the share of firms present on the frontier in year $t$ that were already on the frontier in year $t-1$. These results for Germany are consistent with the growing entrenchment at the frontier reported by Andrews et al. (2019), and with the broader literature on declining business dynamism (Decker et al., 2017, 2016b,c,a). To check for possible entrenchment on the frontier, we compare the degree of persistence in the first two years of the same sample period with the degree of persistence in the final two years of the sample period. ${ }^{25}$

Three results stand out in Table 8. First, persistence at the frontier is high, especially at the end of the observation period. The persistence is more pronounced on the KBC frontier than on the TFP frontier. At the end of the sample period, persistence on the TFP frontier is $77 \%$ in manufacturing and $71 \%$ in services. In contrast, it is $94 \%$ in manufacturing and $77 \%$ in services along the $\mathrm{KBC}$ frontier. This is unsurprising given the stronger persistence in the firms' stocks of KBC. Hence, the composition of firms on the KBC frontier is more stable than on the TFP frontier. Second, persistence is increasing over time. This is especially striking when analysing the change in the persistence of the TFP frontier in services. Third, persistence is higher in manufacturing industries compared to the service sector, which is also in line with Andrews et al. (2019). It should be noted that the low rate of persistence in the services sector is in part driven by the larger amount of resampling, and hence larger share of firms new to the sample in year $t$.

Table 8: Share of firms on the frontier in $t$ that were also on the frontier is $t-1$

\begin{tabular}{l|cc|cc}
\hline \multirow{2}{*}{ Period } & \multicolumn{2}{|c|}{ Manufacturing } & \multicolumn{2}{|c}{ Service } \\
& KBC & TFP & KBC & TFP \\
\hline Beginning of sample period $^{\dagger}$ & 0.91 & 0.72 & 0.81 & 0.56 \\
End of sample period $^{\ddagger}$ & 0.94 & 0.77 & 0.87 & 0.71
\end{tabular}

$\dagger$ : in manufacturing, $t-1=2009$ and $t=2010$; in services, $t-1=2003$ and $t=2004$

¥: in manufacturing, $t-1=2013$ and $t=2014$; in services, $t-1=2012$ and $t=2013$

\subsection{Relationship between $K B C$ use and productivity divergence}

To link our results regarding the effect of $\mathrm{KBC}$ on productivity, obtained in Section 5 with the aggregate patterns observed in Figures 3 and 4, we calculate the productivity gap between the frontier and the rest for each 2-digit industry, and look at the correlation with the elasticity of KBC. The productivity gaps at the 2-digit industry are shown in Appendix Figures D.2 and D.3. Table 9 reports the correlation coefficients between these

\footnotetext{
${ }^{25}$ This implies that the observations period differs between manufacturing and services. In manufacturing, the first two years are 2009 and 2010, and the last two years capture the 2013 to 2014 period. In services, we use the 2003 to 2004 period and the 2012 to 2013 period.
} 
productivity gaps and various measures of the effect of $\mathrm{KBC}$ on productivity. The first line shows the correlation with the average elasticity of $\mathrm{KBC}$ on productivity, $\theta_{c}$, as reported in Tables 3 and 4 . In the following two lines, we use the elasticity of KBC of the frontier group and of the median groups, as plotted in Figures 1 and 2 . In the final five lines, we calculate the difference in the elasticity of $\mathrm{KBC}$ between the following groups: Frontier vs. Average; Frontier vs. Median; Frontier vs. Top quintile; Top quintile vs. Middle quintile; and Middle quintile vs. Bottom quintile.

Table 9: Correlation coefficient between productivity gap and elasticities of KBC

\begin{tabular}{|c|c|c|c|c|c|c|}
\hline & $\begin{array}{l}\text { All } \\
(1)\end{array}$ & $\begin{array}{l}\text { KBC Frontier } \\
\text { Manufacturing } \\
(2)\end{array}$ & $\begin{array}{c}\text { Services } \\
(3)\end{array}$ & $\begin{array}{l}\text { All } \\
(4)\end{array}$ & $\begin{array}{l}\text { TFP Frontier } \\
\text { Manufacturing } \\
(5)\end{array}$ & $\begin{array}{c}\text { Services } \\
(6)\end{array}$ \\
\hline$\theta_{c}^{\text {Average }}$ & 0.234 & $0.440^{*}$ & 0.117 & 0.131 & 0.218 & 0.00255 \\
\hline$\theta_{c}^{\text {Frontier }}$ & $0.329^{* *}$ & $0.523^{* *}$ & 0.227 & 0.130 & 0.284 & -0.0187 \\
\hline$\theta_{c}^{M e d i a n}$ & 0.247 & $0.491^{* *}$ & 0.119 & 0.116 & 0.223 & -0.0141 \\
\hline Diff. $\theta_{c}^{\text {Frontier }}-\theta_{c}^{\text {Average }}$ & $0.428 * *$ & $0.548^{* *}$ & $0.364^{*}$ & 0.105 & 0.284 & -0.0625 \\
\hline Diff. $\theta_{c}^{\text {Frontier }}-\theta_{c}^{\text {Median }}$ & $0.445^{* *}$ & $0.506^{* *}$ & $0.388^{*}$ & 0.135 & 0.286 & -0.0251 \\
\hline Diff. $\theta_{c}^{F r o n t i e r}-\theta_{c}^{Q 1}$ & $0.407^{* *}$ & $0.445^{* *}$ & $0.349^{*}$ & -0.0690 & 0.231 & -0.247 \\
\hline Diff. $\theta_{c}^{Q 1}-\theta_{c}^{M e d i a n}$ & $0.459^{* *}$ & $0.537^{* *}$ & $0.405^{*}$ & $0.282^{*}$ & 0.307 & 0.165 \\
\hline Diff. $\theta_{c}^{\text {Median }}-\theta_{c}^{Q 5}$ & $0.320^{* *}$ & $0.540^{* *}$ & 0.206 & 0.0358 & 0.144 & -0.120 \\
\hline Observations & 44 & 20 & 24 & 44 & 20 & 24 \\
\hline
\end{tabular}

${ }^{*} \mathrm{p}<0.1,{ }^{* *} \mathrm{p}<0.05$. All quantities listed in the first column are correlated with the difference in average growth rate of productivity on the frontier and the average growth rate of productivity of all other firms. For manufacturing sectors, this difference refers to the period 2009 to 2014, for the services sectors it refers to the period 2009 to 2013 . We identify industries C21, C31 and H50 as outliers and they are dropped from the analysis. 

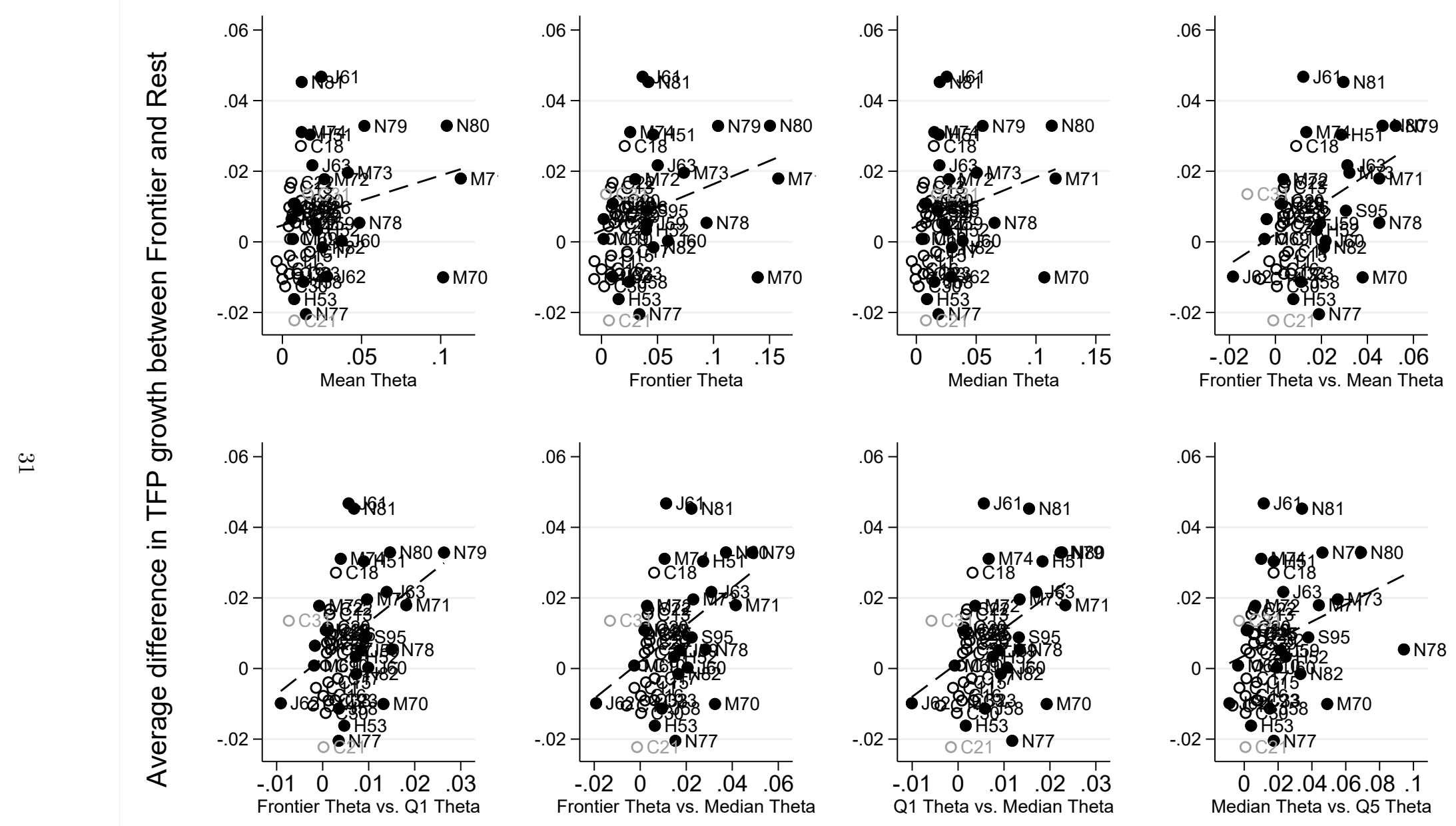

○ Manufacturing $\bullet$ Services ----- Fit 

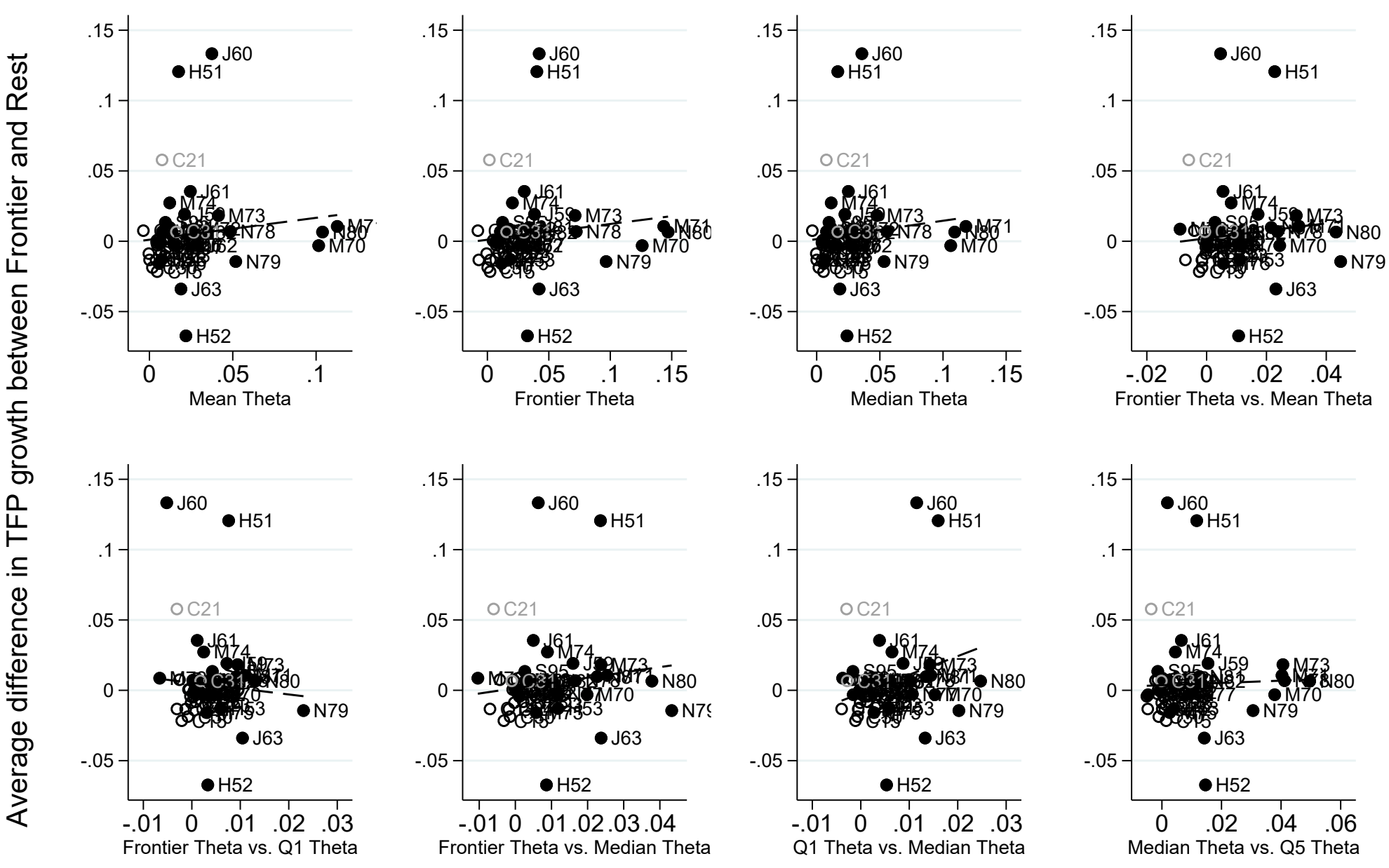

O Manufacturing $\odot$ Services ----- Fit

The unit of observations plotted are 2-digit industries. Industries C21, C31 and H50 are considered outliers, and thus plotted in light gray and not included in the estimation of the fitted line. 
In the first three columns, these different measures of the effect of $\mathrm{KBC}$ on productivity are correlated with the productivity gap on the KBC frontier. We find that the productivity gap is positively correlated with all measures of elasticity. The average and median elasticity of KBC have a small but positive association with the productivity gap, but the correlation of the gap with the elasticity of $\mathrm{KBC}$ on the frontier is very strong, reaching 0.5 in the manufacturing sector and 0.2 in the services sector. This is further strengthened when looking at the difference between the elasticity of $\mathrm{KBC}$ on the frontier with the elasticity of $\mathrm{KBC}$ at the average $(0.55$ in manufacturing, 0.36 in services) or at the median ( 0.51 in manufacturing, 0.39 in services). In the last three columns, we repeat the exercise for the TFP frontier, and find a much weaker correlation between productivity divergence between the frontier and the rest and the effect of $\mathrm{KBC}$ on productivity. The correlation coefficients are around 0.2 in manufacturing, and close to zero in services. This is in line with the results of Section 5 , where we find that the effect of $\mathrm{KBC}$ on productivity does not vary with firm productivity. Figures 5 and 6 display the data behind these correlation coefficients.

Taken together, these results support our hypothesis that $\mathrm{KBC}$ is likely to be a driver of productivity divergence. In industries where the effect of $\mathrm{KBC}$ on productivity is stronger on the frontier than it is for other groups of firms, we observe productivity growth on the frontier to strongly outpace the average productivity growth of the industry. We also find a strong correlation between the productivity gap and differences in the returns to $\mathrm{KBC}$ for the bottom part of the distribution, which suggests that those firms that under-invest in $\mathrm{KBC}$, and that have a negative or zero elasticity of $\mathrm{KBC}$, tend to be at a considerable disadvantage in terms of productivity performance, and might be responsible for pulling the average performance downwards.

\subsection{Divergence across firms and aggregate productivity performance}

In this section, we explore the link between productivity divergence and aggregate productivity performance. In particular, we are interested in understanding whether the strong performance of frontier firms acts as a pull on the industry average, or whether this strong performance comes at the expense of the other firms. To do so, we follow Andrews et al. (2019) and estimate the following relationship.

$$
\Delta_{l} \omega_{j t}^{\text {Total }}=\beta \Delta_{l} G A P_{j t}+\eta_{j}+\eta_{t}+\epsilon_{j} t
$$

$\omega_{j t}^{\text {Total }}$ is aggregate $\log$ productivity of industry $j$ at time $t$, and $G A P_{j t}=\omega_{j t}^{\text {Frontier }}-\omega_{j t}^{\text {Rest }}$ is the level difference between $\log$ productivity on the frontier and $\log$ productivity in the rest of industry $j$ at time $t$. We take the long difference of both of these variables and estimate specifications with difference $l=3,5$. We include industry, $\eta_{j}$, and year, $\eta_{t}$, fixed effects. The coefficient of interest is $\beta$. If it is negative, this means that industries with a faster growth in productivity gap had lower overall productivity growth. This implies that even as the frontier is improving its performance, there are important frictions which hinder the catch-up of non-frontier firms.

The results are reported in Table 10. The first four columns report the results along the KBC frontier, while 
the last four columns report results along the TFP frontier. For each frontier definition, the first three columns are the baseline estimation, using a 3 year lag, and hence can be estimated for both the manufacturing and services sectors, and the aggregate. The estimations are also run using a 5 year lag, which can only be estimated for the service sectors. We consistently find a negative relationship between increases in the productivity gap along the $\mathrm{KBC}$ frontier and the productivity growth of the whole industry, while this relationship is weakly positive on the TFP frontier. This suggests that the over performance in the upper tail of the KBC distribution does not contribute to pulling the industry average upwards. On the contrary, there seems to be an increasing disconnect between firms on the KBC frontier and the rest of the population. We interpret these results as an indication that the increasing use of KBC in the economy plays a role in the aggregate productivity slowdown, by accentuating "best versus rest" dynamics.

Table 10: Change in average aggregate productivity regressed on the change in productivity gap (long differences, 3 and 5 years)

\begin{tabular}{|c|c|c|c|c|c|c|c|c|}
\hline \multirow{3}{*}{$\begin{array}{l}\text { Definition of Frontier } \\
\text { Long difference }\end{array}$} & \multicolumn{4}{|c|}{ KBC Stock } & \multicolumn{4}{|c|}{ TFP Stock } \\
\hline & & 3 years & & 5 years & & 3 years & & 5 years \\
\hline & $\begin{array}{l}\text { ALL } \\
(1)\end{array}$ & $\begin{array}{l}\text { Manuf } \\
(2)\end{array}$ & $\begin{array}{l}\text { Serv } \\
(3)\end{array}$ & $\begin{array}{l}\text { Serv } \\
(4)\end{array}$ & $\begin{array}{l}\text { ALL } \\
(5)\end{array}$ & $\begin{array}{l}\text { Manuf } \\
(6)\end{array}$ & $\begin{array}{l}\text { Serv } \\
(7)\end{array}$ & $\begin{array}{l}\text { Serv } \\
(8)\end{array}$ \\
\hline$\Delta$ Productivity gap & $\begin{array}{c}-0.574^{* * *} \\
(-4.38)\end{array}$ & $\begin{array}{l}0.0501 \\
(0.39)\end{array}$ & $\begin{array}{c}-0.688^{* * *} \\
(-4.43)\end{array}$ & $\begin{array}{c}-0.459^{* *} \\
(-2.77)\end{array}$ & $\begin{array}{l}0.120^{*} \\
(2.40)\end{array}$ & $\begin{array}{c}0.0923 \\
(0.78)\end{array}$ & $\begin{array}{l}0.115^{*} \\
(2.01)\end{array}$ & $\begin{array}{l}0.0361 \\
(0.67)\end{array}$ \\
\hline$R^{2}$ & 0.482 & 0.627 & 0.476 & 0.696 & 0.448 & 0.631 & 0.424 & 0.676 \\
\hline$N$ & 251 & 66 & 185 & 135 & 251 & 66 & 185 & 135 \\
\hline Industry and Year FE & YES & YES & YES & YES & YES & YES & YES & YES \\
\hline
\end{tabular}




\section{Summary}

This study contributes to the discussion around the global slowdown of aggregate productivity growth and the accompanying increase in productivity dispersion between top performers and the rest. Both these developments have been well documented, but their underlying drivers are still not well understood and the subject of ongoing debate, among both academics and economic policy makers. The deceleration of productivity growth is all the more surprising considering that productivity-enhancing investments have grown substantially in recent decades. These investments encompass spending on computerized information, innovative property, and economic competencies, collectively subsumed under the term Knowledge-Based Capital (KBC).

Building on recent developments in the literature on growth through creative destruction, we develop the hypothesis that the relationship between $\mathrm{KBC}$ and productivity, which previous studies found to be positive on average, is in fact heterogeneous across firms. We expect to find strong positive effects at the upper tail of the KBC distribution, and little or no effect at the lower tail. We postulate the additional hypothesis that this heterogeneity in the effect of KBC on productivity exacerbates differences between firms, leading to divergence in productivity, resulting in negative consequences on aggregate productivity growth.

These hypotheses are tested using a dataset with more than one million firm-year observations for Germany. These data cover the entire manufacturing industry, and large parts of the service industry, including most of the so-called knowledge intensive services. ${ }^{26}$ The dataset encompasses the KBC assets R\&D, organizational capital, software and databases, and intellectual property products (IPP), thus capturing $63 \%$ of total investment in KBC assets in Germany (Corrado et al., 2016). The econometric analysis is based on the control function approach of Ackerberg et al. (2015), where KBC is modelled in the law of motion of productivity with a flexible functional form that allows for non-linearities in the relationship.

The estimation results confirm the presence of a non-linear relationship between KBC and productivity. The largest marginal effect of $\mathrm{KBC}$ is found in the group of firms located at the top of the KBC distribution, and decrease in magnitude with the size of $\mathrm{KBC}$. In the bottom quintile of the KBC distribution, the marginal effect is often not different from zero or even negative in a number of industries. In other words, firms that manage to build up a sizeable KBC stock are able to outperform their peers, whereas firms with small or no KBC stock have little incentives to start investing, as these investments will not yield interesting returns. The robustness checks confirm that these results are not sensitive to the use of stocks instead of investment flows, to the assumed functional form of the law of motion, or to the measurement of organisational capital.

In the long run, we expect the heterogeneity in returns to $\mathrm{KBC}$ to exacerbate differences between firms in their ability to invent or absorb new technologies, products, and services, and thereby to improve their productivity. We relate the firm-level estimation results to the evolution of aggregate productivity to test this

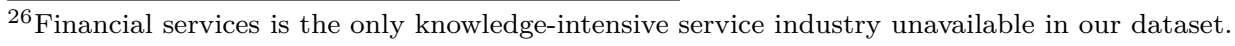


hypothesis. Previous studies have documented, using international data, a growing gap in productivity growth between firms in the top $5 \%$ of the productivity distribution and the rest. Contrary to these studies, we do not find strong evidence that the most productive firms outperform the rest in Germany. However, we also fail to find evidence of catching up of the average firm to the productivity level of the frontier. The comparable productivity growth rates for both frontier and non-frontier firms suggests that initial differences in productivity levels continue to increase between these two groups of firms. Consequently, productivity dispersion between German firms is also increasing, but at a slower rate compared to other countries.

We extend this discussion by exploring the role of $\mathrm{KBC}$ in productivity divergence, and find that firms with the top $5 \%$ largest stocks of KBC have seen faster productivity growth than the population of firms. The gap in productivity growth amounted to 3 percentage points in manufacturing and 9.5 percentage points in services over the observation period. ${ }^{27}$ Additional analyses at the more detailed industry level reveal that the gap in productivity growth between frontier firms and followers is highest in those industries where the difference in the marginal effect of KBC is most pronounced between these groups. Furthermore, we show that industries in which the productivity gap between large KBC firms and the rest was more marked are also those industries in which average productivity growth was lower.

Our analysis thus adds new insights to the debate on the slowdown of productivity growth and the growing divergence between firms. To the best of our knowledge, it is the first to provide robust empirical evidence for the role of KBC in these dynamics. However, the importance of this mechanism in relation to the other discussed drivers of the productivity slowdown remains to be explored. Of particular importance is understanding the interaction between the growing concentration of $\mathrm{KBC}$ investment in a limited number of firms and the role of competition authorities and product-market regulations in affecting the contestability of markets and the development of market power.

\footnotetext{
${ }^{27}$ The observation period differs for manufacturing and services. While the latter covers 2003 to 2013 , the former is restricted to 2009 to 2014. This is due to the fact that information on investments in software and in IPP was not collected by the statistical office for manufacturing firms prior to 2009 .
} 


\section{References}

Ackerberg, D. A., Caves, K., Frazer, G., 2015. Identification properties of recent production function estimators. Econometrica 83 (6), 2411-2451.

Aghion, P., Bergeaud, A., Boppart, T., Klenow, P. J., Li, H., 2019. A theory of falling growth and rising rents. NBER Working Paper No. 26448.

Akcigit, U., Ates, S., 2019a. Ten facts on declining on business dynamics and lessons from endogenous growth theory. NBER Working Paper No. 25755.

Akcigit, U., Ates, S., 2019b. What happened to US business dynamism. NBER Working Paper No. 25756.

Andrews, D., Criscuolo, C., Gal, P., 2015. Frontier firms, technology diffusion and public policy: Micro evidence from OECD countries. OECD Productivity working papers No. 2.

Andrews, D., Criscuolo, C., Gal, P., 2019. The best versus the rest: Divergence across firms during the global productivity slowdown. CEP Discussion Paper 1645.

Autor, D., Dorn, D., Katz, L., Patterson, C., Reenen, J. V., 2020. The fall of the labor share and the rise of superstar firms. Quarterly Journal of Economics 135 (2), 645-709.

Aw, B. Y., Roberts, M. J., Xu, D. Y., 2011. R\&D investment, exporting, and productivity dynamics. American Economic Review 101 (4), 1312-44.

Bahar, D., 2018. The middle productivity trap: Dynamics of productivity dispersion. Economic Letters 167, $60-66$.

Baumann, J., Kritikos, A., 2016. The link between R\&D, innovation and productivity: Are micro firms different? Research Policy 45 (6), 1263-1274.

Benhabib, J., Perla, J., Tonetti, C., 2019. Reconciling models of diffusion and innovation: A theory of the productivity distribution and technology frontier. NBER Working Paper No. 23095.

Berlingieri, G., Blanchenayi, P., Criscuolo, C., 2017. The great divergence(s). OECD Science, Technology and Industry Policy Papers No. 39.

Bersch, J., Diekhof, J., Krieger, B., Licht, G., Murmann, S., 2019. From Industrial Organization to Enterpreneurship. Springer, Ch. Productivity Slowdown, Innovation and Industry Dynamics, pp. 229-242.

Bloom, N., Romer, P. M., Terry, S. J., Van Reenen, J., 2013. A trapped-factors model of innovation. American Economic Review 103 (3), 208-13. 
Bloom, N., Sadun, R., Reenen, J. V., 2017. Management as a technology? NBER Working Paper No. 22327.

Bontempi, M. E., Mairesse, J., 2015. Intangible capital and productivity at the firm level: A panel data assessment. Economics of Innovation and New Technology 24 (1-2), 22-51.

Brynjolfsson, E., Rock, D., Syverson, C., 2020. The productivity J-curve: How intangibles complement General Purpose Technologies. NBER Working Paper No. 25148.

Cardona, M., Kretschmer, T., Strobel, T., 2013. ICT and productivity: Conclusions from the empirical literature. Information economics and policy 25 (3), 109-125.

Cette, G., Corde, S., Lecat, R., 2018. Firm-level productivity dispersion and convergence. Economic Letters 166, $76-78$.

Chappell, N., Jaffe, A., 2018. Intangible investment and firm performance. NBER Working paper series No. 24363.

Chen, W., Inklaar, R., 2015. Productivity spillovers of organization capital. Journal of Productivity Analysis $45,229-245$.

Corrado, C., Haskel, J., Jona-Lasinio, C., Iommi, M., 2016. Investment and investment finance in Europe: Financing productivity growth. European Investment Bank, Ch. Growth, tangible and intangible investment in the EU and US before and since the Great Recession, pp. 73-103.

Corrado, C., Hulten, C., Sichel, D., 2005. Measuring Capital in a New Economy. National Bureau of Economic Research and University of Chicago Press, Ch. Measuring Capital and Technology: An Expanded Framework, pp. 11-46.

Corrado, C., Hulten, C., Sichel, D., 2009. Intangible capital and the US economy. The Review of Income and Wealth $55(3), 661-685$.

Covarrubias, M., Gutiérrez, G., Philippon, T., 2019. From good to bad concentration? U.S. industries over the past 30 years. NBER Working Paper No. 25983.

Crass, D., Peters, B., 2014. Intangible assets and firm-level productivity. ZEW Discussion papers No. 14-120.

Crepon, B., Duguet, E., Mairessec, J., 1998. Research, innovation and productivity: An econometric analysis at the firm level. Economics of Innovation and New Technology 7 (2), 115-158.

Crouzet, N., Eberly, J., 2018. Intangibles, investment, and efficiency. AEA Papers and Proceedings 108, 426-431.

De Loecker, J., Eeckhout, J., Unger, G., 2020. The rise of market power and the macroeconomic implications. Quarterly Journal of Economics 135 (2), 561-644. 
De Loecker, J., Warzynski, F., 2012. Markups and firm-level export status. American Economics Review 102 (6), $2437-2471$.

Decker, R. A., Haltiwanger, J., Jarmin, R. S., Miranda, J., 2016a. Declining Business Dynamism: Implications for Productivity? Brookings Institution Hutchins Center Working Paper No. 23.

Decker, R. A., Haltiwanger, J., Jarmin, R. S., Miranda, J., 2016b. Declining Business Dynamism: What We Know and the Way Forward. American Economic Review 106 (5), 203-207.

Decker, R. A., Haltiwanger, J., Jarmin, R. S., Miranda, J., 2016c. Where has all the skewness gone? The decline in high-growth (young) firms in the U.S. European Economic Review 86 (C), 4-23.

Decker, R. A., Haltiwanger, J., Jarmin, R. S., Miranda, J., 2017. Declining Dynamism, Allocative Efficiency, and the Productivity Slowdown. American Economic Review 107 (5), 322-326.

DeRidder, M., 2019. Market power and innovation in the intangible economy. Mimeo.

Dierks, S., Schiersch, A., Stede, J., 2020. Industry Conversion Tables for German Firm-Level Data. Journal of Economics and Statistics online first.

Doraszelski, U., Jaumandreu, J., 2013. R\&D and productivity: Estimating endogenous productivity. Review of Economic Studies 80 (4), 1338-1383.

Eisfeldt, A., Papanikolaou, D., 2013. Organization capital and the cross-section of expected returns. Journal of Finance 68 (4), 1365-1406.

Faggio, G., Salvanes, K., Reenen, J. V., 2010. The evolution of inequality in productivity and wages: Panel data evidence. Industrial and Corporate Change 19 (6), 1919-1951.

Fritsch, M., Görzig, B., Hennchen, O., Stephan, A., 2004. Cost structure surveys for Germany. Schmollers Jahrbuch: Journal of Applied Social Science Studies/Zeitschrift für Wirtschafts- und Sozialwissenschaften $124(4), 557-566$.

Garcia-Macia, D., Hsieh, C.-T., Klenow, P., 2019. How destructive is innovation? Econometrica.

Griffith, R., Huergo, E., Peters, B., Mairesse, J., 2016. Innovation and productivity across four European countries. Oxford Review of Economic Policy 22 (4), 483-498.

Hall, B. H., 2011. Innovation and productivity. Nordic Economic Policy Review, 167-204.

Hall, B. H., Lotti, F., Mairesse, J., 2009. Innovation and productivity in SMEs: Empirical evidence for Italy. Small Business Economics 33, 13-33. 
Haskel, J., Westlake, S., 2018. Capitalism without Capital: The rise of the intangible economy. Princeton University Press.

Heining, J., Klosterhuber, W., Seth, S., 2014. An overview on the linked employer-employee data of the Institute for Employment Research (IAB). Schmollers Jahrbuch 134 (1), 141-148.

Hsieh, C.-T., Rossi-Hansberg, E., 2019. The industrial revolution in sercices. NBER Working Paper No. 25968.

Kancs, A., Siliverstovs, B., 2016. R\&D and non-linear productivity growth. Research Policy 45 (3), $634-646$.

Kaus, W., Slavtchev, V., Zimmermann, M., 2020. Intangible capital and productivity: Firm-level evidence from German manufacturing. IWH Discussion Papers, No. 1/2020.

Koch, A., 2007. Neue Datenquelle „Unternehmensregister“: Mehr Informationen über den Mittelstand ohne neue Bürokratie. Institut für Mittelstandsforschung. Bonn.

Le Mouel, M., Squicciarini, M., 2015. Cross-country estimates of employment and investment in organisational capital: A task-based methodology using PIAAC data. OECD Science, Technology and Industry Working Papers 2015/8.

Lev, B., Radhakrishnan, S., Zhang, W., 2009. Organization capital. Abacus 45 (3), 275-298.

Levinsohn, J., Petrin, A., 2003. Estimating production functions using inputs to control for unobservables. Review of Economic Studies 70 (2), 317-341.

Marrocu, E., Paci, R., Pontis, M., 2011. Intangible capital and firms' productivity. Industrial and Corporate Change 21 (2), 377-402.

Marschak, J., Andrews Jr., W. H., 1944. Random simultaneous equations and the theory of production. Econometrica $12(3 / 4), 143-205$.

Miyagawa, T., Hisa, S., 2013. Measurement of intangible investment by industry and economic growth in Japan. Public Policy Review 9 (2), 405-432.

Mohnen, P., Polder, M., van Leeuwen, G., 2018. ICT, R\&D and organizational innovation: Exploring complementarities in investment and production. NBER Working Paper No. 25044.

Niebel, T., O'Mahony, M., Saam, M., 2017. The contribution of intangible asssets to sectoral productivity growth in the EU. Review of Income and Wealth 63 (s1), S49-S67.

OECD, 2013. New Sources of Growth: Knowledge-Based Capital - Key Analyses and Policy Conclusions. OECD Publishing. 
OECD, 2015. The Future of Productivity. OECD Publishing, Paris.

Olley, S., Pakes, A., 1996. The dynamics of productivity in the telecommunications equipment industry. Econometrica 64 (6), 1263-1297.

Polder, M., de Bondt, H., van Leeuwen, G., 2018. Business dynamics, industry productivity growth, and the distribution of firm-level performance: Evidence for the role of ICT using Dutch firm-level data. Journal of Technology Transfer 43, 1522-1541.

Richter, P. M., Schiersch, A., 2017. $\mathrm{CO}_{2}$ emission intensity and exporting: Evidence from firm-level data. European Economic Review 98 (C), 373-391.

Schiersch, A., 2019. Frontiers und Laggards die Produktivitätsentwicklung deutscher Unternehmen. Tech. rep., Bertelsmann Stiftung.

Schiersch, A., Schmidt-Ehmcke, J., 2011. Is the Boone-indicator applicable? Evidence from a combined data set of German manufacturing enterprises. Journal of Economics and Statistics 231 (3), 336-357.

Schivardi, F., Schmitz, T., 2019. The IT revolution and Southern Europe's two lost decades. Journal of the European Economic Association.

Siedschlag, I., Lawless, M., Di Ubaldo, M., 2017. The impact of business investment in knowledge-based capital on productivity growth: A review of international and Irish evidence. ESRI Working Paper WP557.

Syverson, C., 2017. Challenges to mismeasurement explanations for the US productivity slowdown. Journal of Economic Perspectives 31 (2), 165-186.

Ugur, M., Trushin, E., Solomon, E., Guidi, F., 2016. R\&D and productivity in OECD firms and industries: A hierarchical meta-regression analysis. Research Policy 45 (10), 2069-2086. 


\section{Appendix A. Description of the datasets}

The three main datasets used in the present analysis are the AFiD Panel of Manufacturing Firms, the AFiD Panel of Service Firms and the Linked Employer-Employee Data of the IAB (LIAB). All the data are representative at the 2-digit industry and size class levels. The AFiD Panels are of very high quality because they serve as the basis for the construction of the National Accounts. Firms are legally required to deliver the requested data, and the Statistical Office ensures that the non-response rate is as low as possible (around $2 \%$ in recent years). The Statistical Office is also responsible for performing quality checks and for checking for implausibilities.

The AFiD Panel of Manufacturing Firms consists of the following three surveys, merged using unique firm identifiers:

- the annual report for manufacturing, mining and quarrying companies (JBU)

- the investment survey of manufacturing, mining and quarrying companies (IEU)

- the cost structure survey in manufacturing, mining and quarrying (KSE)

The AFiD Panel of Service Firms consists of individual surveys for the following sectors: transport and warehousing $(\mathrm{H})$, information and communication $(\mathrm{J})$, provision of professional, scientific and technical services $(\mathrm{M})$, provision of other economic services (N), other services (S95).

We provide a brief description of the coverage and list of variables for each of the sub-datasets, and refer interested readers to Schiersch and Schmidt-Ehmcke (2011); Richter and Schiersch (2017); Fritsch et al. (2004); Koch (2007); Heining et al. (2014) for detailed descriptions of the investment survey, the cost structure survey, the annual reports and the LIAB dataset.

\section{A.1. AFiD-Panel of Manufacturing Firms}

\section{A.1.1. Annual report for manufacturing, mining and quarrying companies}

Coverage. The annual report is a census of all manufacturing, mining and quarrying firms with more than 20 employees. In some industries, such as the food industry or the mining sector, the threshold has been lowered to 10 employees. The dataset contains a total of around 61,000 individual firms, with approximately 22,000 that are observed for the entire sample period of 2003 to 2014, and around 4,800 that are observed only once. The remaining 34,400 are observed between two and eleven years.

List of variables. The analysis uses the following variables from the annual report: number of active persons; payroll; turnover. 


\section{A.1.2. Investment survey of manufacturing, mining and quarrying companies}

Coverage. The investment survey is also a census of all manufacturing, mining and quarrying firms with more than 20 employees and a reduced threshold of 10 employees in a few industries.

List of variables. The analysis uses the following variables from the investment survey: investments in concessions, patents, licenses, trademarks, etc.; investments in software; purchased and self-constructed property, plant and equipment; value of newly leased property, plant and equipment; sales of property, plant and equipment. Note that the variables investment in software and investment in concessions, patents, licenses, trademarks, etc. are included in the investment survey starting in 2009.

\section{A.1.3. Cost structure survey in manufacturing, mining and quarrying}

Coverage. The cost structure survey includes both the full census of firms with at least 500 employees, and a sample for firms below that threshold. Smaller firms are sampled from the full population of manufacturing, mining and quarrying firms recorded in the business register, using a stratification strategy that ensures representativeness at the industry and size class levels. Note that the survey is not representative at the level of federal states. To limit the response burden of firms, a new sample is drawn every four years. However, in industries with few firms, even small firms are part of the sample almost every year. The total number of firms in the cost structure survey covers around $45 \%$ of all firms in the mining and manufacturing industry.

List of variables. The analysis uses the following variables from the cost structure survey: legal form; federal state; industry code; total number of employees; total number of active persons; total turnover; gross production value; gross value added; consumption of raw materials and supplies; gross payroll (without employer contributions); employer payroll taxes; expenditure on $\mathrm{R} \& \mathrm{D}$; correction factors.

\section{A.2. AFiD-Panel of Service Firms}

Coverage. The AFiD Panel of Service Firms is a sample of firms, drawn from the population of firms subject to VAT, with at least $€ 17,500$ of annual turnover, to be representative at the industry, federal state and size class levels. The number of firms included in the sample represent around $15 \%$ of total firms in the relevant services sectors. To minimize the administrative burden on firms, new samples were drawn in 2003, 2008, and 2011. As a result, only 2 percent of the firms in the services dataset are present for the entire sample period of 2003 to 2013. Around 10 percent of the firms are constantly in the surveys between 2003 to 2007 and around 5 percent of all firms are constantly observed in the period 2008 to 2013.

List of variables. The analysis uses the following variables from the AFiD Panel of Service Firms: legal form; federal state; industry code; number if employees; number of all active persons; turnover; gross wages and salaries; consumption of raw materials and supplies; investment in property, plant and equipment; investments in concessions, patents, licenses, trademarks, etc.; investment in software. 


\section{A.3. Linked Employer-Employee Data of the IAB}

Two important asset categories are unavailable in the AFiD Panels: organisational capital for all firms, and $\mathrm{R} \& \mathrm{D}$ for firms in the services sectors. This information is computed using occupational information from a third source, the Linked Employer-Employee Data of the IAB (LIAB). To overcome the legal prohibition on the merger of these two datasets at the firm level, we adopt a two-step methodology inspired from the model of Crepon et al. (1998) (commonly referred to as the CDM model, see Griffith et al., 2016; Hall et al., 2009; Hall, 2011; Baumann and Kritikos, 2016).

The intuition behind the CDM approach is as follows. In a first step, the authors estimate the likelihood of a company to engage in $\mathrm{R} \& \mathrm{D}$ activities. In a second step, all companies above a certain likelihood threshold are attributed an estimated $R \& D$ intensity (i.e. R\&D expenditure relative to employment), which replaces observed values of $R \& D$ intensity. The procedure implies that certain firms that report zero $R \& D$ expenditures are nevertheless given a positive value for R\&D. Crepon et al. (1998) use this procedure for two reasons. Firstly, it solves the selection problem with respect to R\&D choice. Secondly, it addresses the well-known problem of under-reporting of R\&D activities and investment in surveys.

We follow this two-step approach. In the first step, we estimate the likelihood of firms to be engaged in investment in either organisational capital or $\mathrm{R} \& \mathrm{D}$. We do so by estimating probit models at the 2-digit industry level, where the explanatory variables are chosen such that they are available in both the LIAB and the AFiD Panels. The dependent variable is, in the case of organisational capital, a dummy for observing at least one manager with an employee relationship to the firm. ${ }^{28}$ In the case of $R \& D$, the dependent variable is a dummy for engaging in R\&D activities, which is reported in the LIAB dataset. We apply the estimated coefficients of the probit estimations to predict the likelihood of firms in the AFiD Panels to invest in the two assets. The calculations are calibrated such that the share of firms engaged in investment in the AFiD Panels matches the one observed in the LIAB dataset, at the 1-digit industry level.

In the second step, for each firm predicted to participate in organisational capital and R\&D investment, we calculate the compensation paid to managerial and R\&D occupations. We identify the relevant occupations from the KldB 2010, the German pendant to ISCO 2008. Their monthly wages are reported in the LIAB data. We calculate the share of managerial and $R \& D$ wages in total wagebill for each firm. To transfer this information to the AFiD Panels, we construct tables by 2-digit industry, geographical region and biennium, where each cell reports the average wage share of the relevant occupations. These tables are applied to the AFiD Panels to calculate the firm-specific compensation of managers and $R \& D$ workers by multiplying these shares with the firm wagebill.

Finally, the estimated compensations are considered investment in the relevant asset and capitalised as

\footnotetext{
${ }^{28}$ Many firms do not hire professional managers and are instead managed by their owners. The presence of an owner manager is
} included as one of the explanatory variables in the probit model. 
described in Section A.4. Note that only $20 \%$ of managerial compensation is considered investment (following Corrado et al., 2009), whereas $100 \%$ of R\&D worker compensation is considered investment.

\section{A.4. Constructing stocks of Knowledge-Based Capital}

Following the intuition of Corrado et al. (2005, 2009), we account for the fact that expenditures on knowledgebased assets benefit firms over multiple years and have a cumulative effect. We assume that total KBC stock, rather than yearly investment flows, improve firm productivity. We apply the perpetual inventory method (PIM) to estimate the tangible capital stocks and the capital stocks of the four $\mathrm{KBC}$ assets.

We make the following additional assumptions regarding deflators, depreciation rates and initial capital stocks. The price deflators for value-added, material expenditure, and investments in tangible capital, software, R\&D and other IPP, are taken from the official series provided by the statistical office, by 2-digit industry and year for each asset. The investment in organisational capital is deflated using the Consumer Price Index. Depreciation rates for R\&D, software, IPP and organisational capital are obtained from the OECD (2013), and depreciation rates for tangible capital, by 2-digit industry, from the national accounts. Finally, initial capital stocks for the KBC assets are obtained from the steady state assumption, namely as the ratio of investment over the depreciation rate. We divide a firm's minimum reported value of investment by the depreciation rate of the year the firm enters the dataset. For physical capital, we adopt a more robust approach, and take the average between two values of initial capital stock. The first value is obtained from the steady state assumption, and the second is the product of the industry capital-labour ratio (provided by the statistical office) with the firm's total labour.

\section{A.5. Additional descriptive tables}

Table A.1 presents the list of industries included in the analysis, along with the industry codes and description, according to the German classification of industries WZ 2008. Tables A.2 and A.3 provide the descriptive statistics of the production variables by 2 -digit industry. 
Table A.1: 2-digit industry classification

\begin{tabular}{|c|c|c|c|}
\hline & (WZ 2008) & Industry code & Description \\
\hline \multirow{22}{*}{ 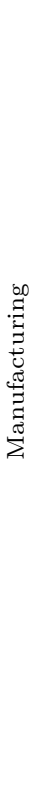 } & & C10 & Food Products \\
\hline & & C11 & Beverages \\
\hline & & $\mathrm{C} 13$ & Textiles \\
\hline & & $\mathrm{C} 14$ & Wearing apparel \\
\hline & & C15 & Leather and related products \\
\hline & & $\mathrm{C} 16$ & $\begin{array}{l}\text { Wood and of products of wood and cork, except furniture; } \\
\text { manufacture of articles of straw and plaiting materials }\end{array}$ \\
\hline & & $\mathrm{C} 17$ & Paper and paper products \\
\hline & & $\mathrm{C} 18$ & Printing and reproduction of recorded media \\
\hline & & $\mathrm{C} 20$ & Chemicals and chemical products \\
\hline & & $\mathrm{C} 21$ & Basic pharmaceutical products and pharmaceutical preparations \\
\hline & & $\mathrm{C} 22$ & Rubber and plastics products \\
\hline & & $\mathrm{C} 23$ & Other non-metallic mineral products \\
\hline & & $\mathrm{C} 24$ & Basic metals \\
\hline & & $\mathrm{C} 25$ & Fabricated metal products, except machinery and equipment \\
\hline & & $\mathrm{C} 26$ & Computer, electronic and optical products \\
\hline & & $\mathrm{C} 27$ & Electrical equipment \\
\hline & & $\mathrm{C} 28$ & Machinery and equipment n.e.c. \\
\hline & & $\mathrm{C} 29$ & Motor vehicles, trailers and semi-trailers \\
\hline & & C30 & Other transport equipment \\
\hline & & C31 & Furniture \\
\hline & & $\mathrm{C} 32$ & Other manufacturing \\
\hline & & C33 & Repair and installation of machinery and equipment \\
\hline \multirow{5}{*}{ 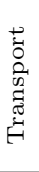 } & \multirow{5}{*}{ 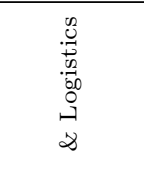 } & $\mathrm{H} 49$ & Land transport and transport via pipelines \\
\hline & & $\mathrm{H} 50$ & Water transport \\
\hline & & H51 & Air transport \\
\hline & & $\mathrm{H} 52$ & Warehousing and support activities for transportation \\
\hline & & H53 & Postal and courier activities \\
\hline \multirow{6}{*}{ 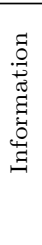 } & \multirow{6}{*}{ 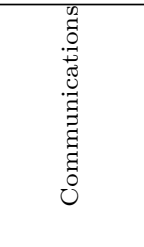 } & J58 & Publishing activities \\
\hline & & J59 & $\begin{array}{l}\text { Motion picture, video and television programme production, } \\
\text { sound recording and music publishing activities }\end{array}$ \\
\hline & & J60 & Programming and broadcasting activities \\
\hline & & J61 & Telecommunications \\
\hline & & J62 & Computer programming, consultancy and related activities \\
\hline & & J63 & Information service activities \\
\hline \multirow{7}{*}{ 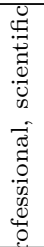 } & \multirow{7}{*}{ 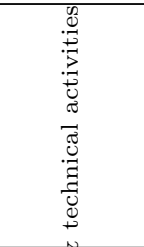 } & M69 & Legal and accounting activities \\
\hline & & M70 & Activities of head offices; management consultancy activities \\
\hline & & M71 & Architectural and engineering activities; technical testing and analysis \\
\hline & & M72 & Scientific research and development \\
\hline & & M73 & Advertising and market research \\
\hline & & M74 & Other professional, scientific and technical activities \\
\hline & & M75 & Veterinary activities \\
\hline \multirow{7}{*}{ 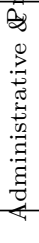 } & \multirow{7}{*}{ 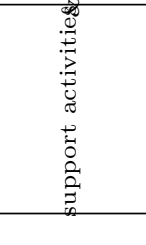 } & N77 & Rental and leasing activities \\
\hline & & N78 & Employment activities \\
\hline & & N79 & Travel agency, tour operator, reservation service and related activities \\
\hline & & N80 & Security and investigation activities \\
\hline & & N81 & Services to buildings and landscape activities \\
\hline & & N82 & Office administrative, office support and other business support activities \\
\hline & & S95 & Repair of computers and personal and household goods \\
\hline
\end{tabular}


Table A.2: Descriptive statistics by 2-digit industry in Manufacturing

\begin{tabular}{|c|c|c|c|c|c|c|c|c|}
\hline & VA & Labour $^{\dagger}$ & Capital & Materials & $\begin{array}{l}\text { KBC } \\
\text { Stock }\end{array}$ & $\begin{array}{l}\text { KBC } \\
\text { Investment }\end{array}$ & $\begin{array}{l}\text { KBC } \\
\text { Intensity }\end{array}$ & $\mathrm{N}$ \\
\hline $\mathrm{C} 10$ & $\begin{array}{l}9.67 \\
(24.2)\end{array}$ & $\begin{array}{l}202 \\
(363.9)\end{array}$ & $\begin{array}{l}23.81 \\
(65.1)\end{array}$ & $\begin{array}{l}33.58 \\
(108.2)\end{array}$ & $\begin{array}{l}1.26 \\
(5.7)\end{array}$ & $\begin{array}{l}.47 \\
(2.1)\end{array}$ & $\begin{array}{l}.04 \\
(.1)\end{array}$ & 12662 \\
\hline C11 & $\begin{array}{l}18.31 \\
(46.3)\end{array}$ & $\begin{array}{l}175 \\
(669.8)\end{array}$ & $\begin{array}{l}43.57 \\
(129.3)\end{array}$ & $\begin{array}{l}20.44 \\
(69.4)\end{array}$ & $\begin{array}{l}1.42 \\
(5.9)\end{array}$ & $\begin{array}{l}.61 \\
(2.5)\end{array}$ & $\begin{array}{l}.03 \\
(0)\end{array}$ & 1715 \\
\hline $\mathrm{C} 13$ & $\begin{array}{l}5.89 \\
(12.2)\end{array}$ & $\begin{array}{l}113 \\
(144.9)\end{array}$ & $\begin{array}{l}15.23 \\
(26.9)\end{array}$ & $\begin{array}{l}9.38 \\
(20)\end{array}$ & $\begin{array}{l}1.08 \\
(4.2)\end{array}$ & $\begin{array}{l}.33 \\
(.8)\end{array}$ & $\begin{array}{l}.05 \\
(.1)\end{array}$ & 2616 \\
\hline C14 & $\begin{array}{l}8.25 \\
(23.1)\end{array}$ & $\begin{array}{l}138 \\
(261.2)\end{array}$ & $\begin{array}{l}13.12 \\
(28.5)\end{array}$ & $\begin{array}{l}10.78 \\
(26.2)\end{array}$ & $\begin{array}{l}1.08 \\
(3.7)\end{array}$ & $\begin{array}{l}.4 \\
(1.5)\end{array}$ & $\begin{array}{l}.06 \\
(.1)\end{array}$ & 1375 \\
\hline $\mathrm{C} 15$ & $\begin{array}{l}5.18 \\
(6.8)\end{array}$ & $\begin{array}{l}108 \\
(110.9)\end{array}$ & $\begin{array}{l}10.14 \\
(12.4)\end{array}$ & $\begin{array}{l}9.07 \\
(17.6)\end{array}$ & $\begin{array}{l}.74 \\
(2.1)\end{array}$ & $\begin{array}{l}.26 \\
(.7)\end{array}$ & $\begin{array}{l}.04 \\
(.1)\end{array}$ & 612 \\
\hline C16 & $\begin{array}{l}5.87 \\
(9.8)\end{array}$ & $\begin{array}{l}112 \\
(157.7)\end{array}$ & $\begin{array}{l}14.92 \\
(43.9)\end{array}$ & $\begin{array}{l}15.52 \\
(32.6)\end{array}$ & $\begin{array}{l}.55 \\
(1.6)\end{array}$ & $\begin{array}{l}.2 \\
(.5)\end{array}$ & $\begin{array}{l}.03 \\
(0)\end{array}$ & 2781 \\
\hline C17 & $\begin{array}{l}14.8 \\
(27.5)\end{array}$ & $\begin{array}{l}212 \\
(347.7)\end{array}$ & $\begin{array}{l}44.09 \\
(91.5)\end{array}$ & $\begin{array}{l}32.03 \\
(63.3)\end{array}$ & $\begin{array}{l}1.48 \\
(3.8)\end{array}$ & $\begin{array}{l}.52 \\
(1.2)\end{array}$ & $\begin{array}{l}.03 \\
(0)\end{array}$ & 3005 \\
\hline C18 & $\begin{array}{l}8.12 \\
(15.2)\end{array}$ & $\begin{array}{l}140 \\
(203.5)\end{array}$ & $\begin{array}{l}15.9 \\
(28.1)\end{array}$ & $\begin{array}{l}9.96 \\
(24.1)\end{array}$ & $\begin{array}{l}.94 \\
(8.1)\end{array}$ & $\begin{array}{l}.33 \\
(2.1)\end{array}$ & $\begin{array}{l}.04 \\
(.1)\end{array}$ & 2334 \\
\hline $\mathrm{C} 20$ & $\begin{array}{l}34.65 \\
(182.7)\end{array}$ & $\begin{array}{l}319 \\
(1405.2)\end{array}$ & $\begin{array}{l}106.76 \\
(482.9)\end{array}$ & $\begin{array}{l}59 \\
(251.7)\end{array}$ & $\begin{array}{l}22.62 \\
(205)\end{array}$ & $\begin{array}{l}5.25 \\
(46.5)\end{array}$ & $\begin{array}{l}.1 \\
(.3)\end{array}$ & 5521 \\
\hline $\mathrm{C} 21$ & $\begin{array}{l}78.66 \\
(318.7)\end{array}$ & $\begin{array}{l}549 \\
(1531.8)\end{array}$ & $\begin{array}{l}198.99 \\
(639.1)\end{array}$ & $\begin{array}{l}47.48 \\
(112.9)\end{array}$ & $\begin{array}{l}80.03 \\
(464.4)\end{array}$ & $\begin{array}{l}17.73 \\
(109.9)\end{array}$ & $\begin{array}{l}.14 \\
(.4)\end{array}$ & 1210 \\
\hline $\mathrm{C} 22$ & $\begin{array}{l}14.37 \\
(40.8)\end{array}$ & $\begin{array}{l}223 \\
(516.6)\end{array}$ & $\begin{array}{l}26.61 \\
(72.8)\end{array}$ & $\begin{array}{l}20.08 \\
(51.1)\end{array}$ & $\begin{array}{l}4.14 \\
(28.9)\end{array}$ & $\begin{array}{l}1.04 \\
(6.4)\end{array}$ & $\begin{array}{l}.06 \\
(.1)\end{array}$ & 6300 \\
\hline $\mathrm{C} 23$ & $\begin{array}{l}12.32 \\
(25.3)\end{array}$ & $\begin{array}{l}181 \\
(297.2)\end{array}$ & $\begin{array}{l}29.87 \\
(56.7)\end{array}$ & $\begin{array}{l}14.32 \\
(27.8)\end{array}$ & $\begin{array}{l}2.37 \\
(9.8)\end{array}$ & $\begin{array}{l}.72 \\
(2.5)\end{array}$ & $\begin{array}{l}.06 \\
(.1)\end{array}$ & 4981 \\
\hline $\mathrm{C} 24$ & $\begin{array}{l}23.92 \\
(81.6)\end{array}$ & $\begin{array}{l}319 \\
(937.3)\end{array}$ & $\begin{array}{l}60.52 \\
(218.3)\end{array}$ & $\begin{array}{l}83.73 \\
(349.8)\end{array}$ & $\begin{array}{l}3.34 \\
(25.2)\end{array}$ & $\begin{array}{l}.85 \\
(4.9)\end{array}$ & $\begin{array}{l}.03 \\
(.1)\end{array}$ & 4238 \\
\hline $\mathrm{C} 25$ & $\begin{array}{l}9.5 \\
(19)\end{array}$ & $\begin{array}{l}151 \\
(255.8)\end{array}$ & $\begin{array}{l}14.9 \\
(30.9)\end{array}$ & $\begin{array}{l}11.17 \\
(27)\end{array}$ & $\begin{array}{l}2.01 \\
(11.3)\end{array}$ & $\begin{array}{l}.61 \\
(2.8)\end{array}$ & $\begin{array}{l}.06 \\
(.1)\end{array}$ & 14426 \\
\hline $\mathrm{C} 26$ & $\begin{array}{l}23.23 \\
(80.7)\end{array}$ & $\begin{array}{l}252 \\
(761.6)\end{array}$ & $\begin{array}{l}44.84 \\
(229.7)\end{array}$ & $\begin{array}{l}24.42 \\
(95.6)\end{array}$ & $\begin{array}{l}22.72 \\
(144)\end{array}$ & $\begin{array}{l}5.02 \\
(26.1)\end{array}$ & $\begin{array}{l}.34 \\
(.6)\end{array}$ & 5030 \\
\hline $\mathrm{C} 27$ & $\begin{array}{l}27.34 \\
(329.8)\end{array}$ & $\begin{array}{l}353 \\
(3501.6)\end{array}$ & $\begin{array}{l}35.26 \\
(284.4)\end{array}$ & $\begin{array}{l}31.38 \\
(271.9)\end{array}$ & $\begin{array}{l}23.78 \\
(428.5)\end{array}$ & $\begin{array}{l}5.07 \\
(83.9)\end{array}$ & $\begin{array}{l}.19 \\
(.3)\end{array}$ & 6868 \\
\hline $\mathrm{C} 28$ & $\begin{array}{l}21.84 \\
(114.9)\end{array}$ & $\begin{array}{l}294 \\
(1471.6)\end{array}$ & $\begin{array}{l}33.31 \\
(232.7)\end{array}$ & $\begin{array}{l}29.56 \\
(140.2)\end{array}$ & $\begin{array}{l}14.64 \\
(265.6)\end{array}$ & $\begin{array}{l}3.56 \\
(61.9)\end{array}$ & $\begin{array}{l}.16 \\
(.3)\end{array}$ & 15717 \\
\hline C29 & $\begin{array}{l}106.14 \\
(880)\end{array}$ & $\begin{array}{l}1099 \\
(8000.7)\end{array}$ & $\begin{array}{l}201.33 \\
(1640.4)\end{array}$ & $\begin{array}{l}265.82 \\
(2242.5)\end{array}$ & $\begin{array}{l}126.87 \\
(1249.7)\end{array}$ & $\begin{array}{l}29.18 \\
(290.5)\end{array}$ & $\begin{array}{l}.17 \\
(.5)\end{array}$ & 3984 \\
\hline C30 & $\begin{array}{l}43.45 \\
(169.1)\end{array}$ & $\begin{array}{l}522 \\
(1756.2)\end{array}$ & $\begin{array}{l}76.26 \\
(367.2)\end{array}$ & $\begin{array}{l}74.3 \\
(328.5)\end{array}$ & $\begin{array}{l}55.57 \\
(455.9)\end{array}$ & $\begin{array}{l}12.31 \\
(93.7)\end{array}$ & $\begin{array}{l}.17 \\
(.3)\end{array}$ & 1294 \\
\hline C31 & $\begin{array}{l}8.49 \\
(21.3)\end{array}$ & $\begin{array}{l}157 \\
(264.8)\end{array}$ & $\begin{array}{l}12.79 \\
(35.4)\end{array}$ & $\begin{array}{l}12.65 \\
(33.1)\end{array}$ & $\begin{array}{l}2.42 \\
(18.4)\end{array}$ & $\begin{array}{l}.69 \\
(4.8)\end{array}$ & $\begin{array}{l}.08 \\
(.1)\end{array}$ & 2557 \\
\hline C32 & $\begin{array}{l}11.57 \\
(35.7)\end{array}$ & $\begin{array}{l}166 \\
(383.1)\end{array}$ & $\begin{array}{l}18.86 \\
(75.8)\end{array}$ & $\begin{array}{l}8.38 \\
(31.3)\end{array}$ & $\begin{array}{l}5.68 \\
(27.6)\end{array}$ & $\begin{array}{l}1.61 \\
(7.7)\end{array}$ & $\begin{array}{l}.15 \\
(.3)\end{array}$ & 4091 \\
\hline C33 & $\begin{array}{l}11.77 \\
(50.1)\end{array}$ & $\begin{array}{l}180 \\
(606.9)\end{array}$ & $\begin{array}{l}7.82 \\
(28.6)\end{array}$ & $\begin{array}{l}12.71 \\
(74.7)\end{array}$ & $\begin{array}{l}3.68 \\
(38.8)\end{array}$ & $\begin{array}{l}.9 \\
(7.3)\end{array}$ & $\begin{array}{l}.19 \\
(1.1)\end{array}$ & 4304 \\
\hline
\end{tabular}

Standard deviation in parentheses. Monetary values in million $€{ }^{\dagger}{ }^{\dagger}$ : head-count number of employees.

$\ddagger$ : KBC Intensity is defined as the the ratio of KBC stock over physical capital stock. 
Table A.3: Descriptive statistics by 2-digit industry in Services

\begin{tabular}{|c|c|c|c|c|c|c|c|c|}
\hline & VA & Labour $^{\dagger}$ & Capital & Materials & $\begin{array}{l}\text { KBC } \\
\text { Stock }\end{array}$ & $\begin{array}{l}\text { KBC } \\
\text { Investment }\end{array}$ & $\begin{array}{l}\text { KBC } \\
\text { Intensity }\end{array}$ & $\mathrm{N}$ \\
\hline H49 & $\begin{array}{l}2 \\
(28)\end{array}$ & $\begin{array}{l}42 \\
(393)\end{array}$ & $\begin{array}{l}9.23 \\
(129.6)\end{array}$ & $\begin{array}{l}3.09 \\
(54.1)\end{array}$ & $\begin{array}{l}.64 \\
(16.5)\end{array}$ & $\begin{array}{l}.14 \\
(3.7)\end{array}$ & $\begin{array}{l}.01 \\
(.4)\end{array}$ & 109499 \\
\hline $\mathrm{H} 50$ & $\begin{array}{l}3.5 \\
(26.9)\end{array}$ & $\begin{array}{l}23 \\
(114)\end{array}$ & $\begin{array}{l}21.7 \\
(161.5)\end{array}$ & $\begin{array}{l}12.3 \\
(184.1)\end{array}$ & $\begin{array}{l}.49 \\
(6)\end{array}$ & $\begin{array}{l}.11 \\
(1.5)\end{array}$ & $\begin{array}{l}.06 \\
(1.5)\end{array}$ & 10628 \\
\hline $\mathrm{H} 51$ & $\begin{array}{l}18.23 \\
(78.4)\end{array}$ & $\begin{array}{l}121 \\
(486.4)\end{array}$ & $\begin{array}{l}31.88 \\
(132.5)\end{array}$ & $\begin{array}{l}43.39 \\
(212.5)\end{array}$ & $\begin{array}{l}6.37 \\
(30)\end{array}$ & $\begin{array}{l}1.38 \\
(6.8)\end{array}$ & $\begin{array}{l}.07 \\
(.3)\end{array}$ & 2047 \\
\hline $\mathrm{H} 52$ & $\begin{array}{l}6.24 \\
(58.8)\end{array}$ & $\begin{array}{l}95 \\
(767.4)\end{array}$ & $\begin{array}{l}53.14 \\
(1490.8)\end{array}$ & $\begin{array}{l}10.92 \\
(69.5)\end{array}$ & $\begin{array}{l}1.86 \\
(31.2)\end{array}$ & $\begin{array}{l}.42 \\
(7.2)\end{array}$ & $\begin{array}{l}.04 \\
(.7)\end{array}$ & 44767 \\
\hline $\mathrm{H} 53$ & $\begin{array}{l}10.68 \\
(266.1)\end{array}$ & $\begin{array}{l}335 \\
(5637.3)\end{array}$ & $\begin{array}{l}10.53 \\
(203.7)\end{array}$ & $\begin{array}{l}11.48 \\
(191.6)\end{array}$ & $\begin{array}{l}.59 \\
(14.5)\end{array}$ & $\begin{array}{l}.23 \\
(6.4)\end{array}$ & $\begin{array}{l}.05 \\
(.4)\end{array}$ & 11962 \\
\hline J58 & $\begin{array}{l}7.62 \\
(34.7)\end{array}$ & $\begin{array}{l}107 \\
(653.9)\end{array}$ & $\begin{array}{l}4.1 \\
(21)\end{array}$ & $\begin{array}{l}7.16 \\
(37.9)\end{array}$ & $\begin{array}{l}1.32 \\
(9.2)\end{array}$ & $\begin{array}{l}.31 \\
(2.6)\end{array}$ & $\begin{array}{l}.6 \\
(3.3)\end{array}$ & 15572 \\
\hline J59 & $\begin{array}{l}2.13 \\
(16.1)\end{array}$ & $\begin{array}{l}27 \\
(78.9)\end{array}$ & $\begin{array}{l}3.05 \\
(13.9)\end{array}$ & $\begin{array}{l}2.74 \\
(12.1)\end{array}$ & $\begin{array}{l}.55 \\
(7.8)\end{array}$ & $\begin{array}{l}.13 \\
(1.7)\end{array}$ & $\begin{array}{l}.47 \\
(6.4)\end{array}$ & 9080 \\
\hline $\mathrm{J} 60$ & $\begin{array}{l}31.6 \\
(148.3)\end{array}$ & $\begin{array}{l}169 \\
(666.6)\end{array}$ & $\begin{array}{l}21.08 \\
(86.9)\end{array}$ & $\begin{array}{l}37.04 \\
(140.4)\end{array}$ & $\begin{array}{l}18.35 \\
(144.7)\end{array}$ & $\begin{array}{l}3.76 \\
(29.1)\end{array}$ & $\begin{array}{l}16.7 \\
(334.1)\end{array}$ & 1185 \\
\hline J61 & $\begin{array}{l}41.91 \\
(444.3)\end{array}$ & $\begin{array}{l}278 \\
(3664.6)\end{array}$ & $\begin{array}{l}182.43 \\
(1953.7)\end{array}$ & $\begin{array}{l}68.9 \\
(634.1)\end{array}$ & $\begin{array}{l}14.18 \\
(150.6)\end{array}$ & $\begin{array}{l}3.9 \\
(59.5)\end{array}$ & $\begin{array}{l}.13 \\
(3.3)\end{array}$ & 6180 \\
\hline J62 & $\begin{array}{l}4.47 \\
(47.2)\end{array}$ & $\begin{array}{l}48 \\
(311.6)\end{array}$ & $\begin{array}{l}3.01 \\
(50.9)\end{array}$ & $\begin{array}{l}5.06 \\
(76.9)\end{array}$ & $\begin{array}{l}1.47 \\
(18.4)\end{array}$ & $\begin{array}{l}.39 \\
(5.2)\end{array}$ & $\begin{array}{l}.81 \\
(6.8)\end{array}$ & 62871 \\
\hline J63 & $\begin{array}{l}2.84 \\
(19.5)\end{array}$ & $\begin{array}{l}53 \\
(438.9)\end{array}$ & $\begin{array}{l}4.33 \\
(52.4)\end{array}$ & $\begin{array}{l}2.91 \\
(27)\end{array}$ & $\begin{array}{l}1.23 \\
(10.6)\end{array}$ & $\begin{array}{l}.23 \\
(2.2)\end{array}$ & $\begin{array}{l}.9 \\
(10.7)\end{array}$ & 16901 \\
\hline M69 & $\begin{array}{l}1.3 \\
(15.1)\end{array}$ & $\begin{array}{l}19 \\
(143.3)\end{array}$ & $\begin{array}{l}.8 \\
(6.5)\end{array}$ & $\begin{array}{l}.5 \\
(7.2)\end{array}$ & $\begin{array}{l}.46 \\
(17.8)\end{array}$ & $\begin{array}{l}.1 \\
(4)\end{array}$ & $\begin{array}{l}.04 \\
(.9)\end{array}$ & 118817 \\
\hline M70 & $\begin{array}{l}3.5 \\
(22.6)\end{array}$ & $\begin{array}{l}32 \\
(148.2)\end{array}$ & $\begin{array}{l}5.19 \\
(52.6)\end{array}$ & $\begin{array}{l}5.14 \\
(147.4)\end{array}$ & $\begin{array}{l}5.28 \\
(30.8)\end{array}$ & $\begin{array}{l}1.2 \\
(7.6)\end{array}$ & $\begin{array}{l}3.72 \\
(57.2)\end{array}$ & 51094 \\
\hline M71 & $\begin{array}{l}1.81 \\
(11.3)\end{array}$ & $\begin{array}{l}24 \\
(122)\end{array}$ & $\begin{array}{l}.94 \\
(7.6)\end{array}$ & $\begin{array}{l}1.53 \\
(15)\end{array}$ & $\begin{array}{l}1.92 \\
(12.4)\end{array}$ & $\begin{array}{l}.44 \\
(3.2)\end{array}$ & $\begin{array}{l}2.52 \\
(10.4)\end{array}$ & 89018 \\
\hline M72 & $\begin{array}{l}4.3 \\
(35.2)\end{array}$ & $\begin{array}{l}73 \\
(600.3)\end{array}$ & $\begin{array}{l}20.07 \\
(181.9)\end{array}$ & $\begin{array}{l}3.25 \\
(20.8)\end{array}$ & $\begin{array}{l}.37 \\
(2.8)\end{array}$ & $\begin{array}{l}.13 \\
(1.2)\end{array}$ & $\begin{array}{l}.08 \\
(1.1)\end{array}$ & 10354 \\
\hline M73 & $\begin{array}{l}1.64 \\
(8.3)\end{array}$ & $\begin{array}{l}38 \\
(242.9)\end{array}$ & $\begin{array}{l}.87 \\
(12.5)\end{array}$ & $\begin{array}{l}2.38 \\
(11.6)\end{array}$ & $\begin{array}{l}1.29 \\
(5.7)\end{array}$ & $\begin{array}{l}.29 \\
(1.4)\end{array}$ & $\begin{array}{l}3.23 \\
(11.7)\end{array}$ & 38105 \\
\hline M74 & $\begin{array}{l}.83 \\
(10.5)\end{array}$ & $\begin{array}{l}13 \\
(91.7)\end{array}$ & $\begin{array}{l}.91 \\
(10.1)\end{array}$ & $\begin{array}{l}.96 \\
(18.3)\end{array}$ & $\begin{array}{l}.43 \\
(12.1)\end{array}$ & $\begin{array}{l}.1 \\
(3.4)\end{array}$ & $\begin{array}{l}.17 \\
(2)\end{array}$ & 26542 \\
\hline M75 & $\begin{array}{l}.3 \\
(.5)\end{array}$ & $\begin{array}{l}7 \\
(10)\end{array}$ & $\begin{array}{l}.3 \\
(.6)\end{array}$ & $\begin{array}{l}.22 \\
(.5)\end{array}$ & $\begin{array}{l}.01 \\
(.1)\end{array}$ & $\begin{array}{l}0 \\
(0)\end{array}$ & $\begin{array}{l}.01 \\
(.1)\end{array}$ & 16666 \\
\hline N77 & $\begin{array}{l}4.01 \\
(37.7)\end{array}$ & $\begin{array}{l}21 \\
(104.7)\end{array}$ & $\begin{array}{l}35.44 \\
(239.9)\end{array}$ & $\begin{array}{l}3.27 \\
(31.4)\end{array}$ & $\begin{array}{l}.72 \\
(8.6)\end{array}$ & $\begin{array}{l}.17 \\
(2.7)\end{array}$ & $\begin{array}{l}.07 \\
(1.7)\end{array}$ & 28031 \\
\hline N78 & $\begin{array}{l}5.85 \\
(33.7)\end{array}$ & $\begin{array}{l}232 \\
(1335.6)\end{array}$ & $\begin{array}{l}4.27 \\
(374.8)\end{array}$ & $\begin{array}{l}1.54 \\
(48.5)\end{array}$ & $\begin{array}{l}.46 \\
(13.8)\end{array}$ & $\begin{array}{l}.09 \\
(1.7)\end{array}$ & $\begin{array}{l}.98 \\
(37.1)\end{array}$ & 23533 \\
\hline N79 & $\begin{array}{l}2.09 \\
(29.7)\end{array}$ & $\begin{array}{l}22 \\
(133.7)\end{array}$ & $\begin{array}{l}1.54 \\
(26.5)\end{array}$ & $\begin{array}{l}6.95 \\
(100)\end{array}$ & $\begin{array}{l}.49 \\
(7)\end{array}$ & $\begin{array}{l}.11 \\
(1.7)\end{array}$ & $\begin{array}{l}.13 \\
(.6)\end{array}$ & 20888 \\
\hline N80 & $\begin{array}{l}3.08 \\
(8.1)\end{array}$ & $\begin{array}{l}133 \\
(298.8)\end{array}$ & $\begin{array}{l}2.58 \\
(33.8)\end{array}$ & $\begin{array}{l}.88 \\
(2.7)\end{array}$ & $\begin{array}{l}.27 \\
(1.2)\end{array}$ & $\begin{array}{l}.1 \\
(.3)\end{array}$ & $\begin{array}{l}16.99 \\
(856.3)\end{array}$ & 9784 \\
\hline N81 & $\begin{array}{l}1.38 \\
(5.9)\end{array}$ & $\begin{array}{l}93 \\
(383.2)\end{array}$ & $\begin{array}{l}4.07 \\
(89.8)\end{array}$ & $\begin{array}{l}.62 \\
(3.7)\end{array}$ & $\begin{array}{l}.13 \\
(.7)\end{array}$ & $\begin{array}{l}.05 \\
(.3)\end{array}$ & $\begin{array}{l}.1 \\
(3.7)\end{array}$ & 72869 \\
\hline N82 & $\begin{array}{l}2.73 \\
(14.1)\end{array}$ & $\begin{array}{l}65 \\
(341.9)\end{array}$ & $\begin{array}{l}4.06 \\
(40.8)\end{array}$ & $\begin{array}{l}3.48 \\
(21.1)\end{array}$ & $\begin{array}{l}1.01 \\
(7.7)\end{array}$ & $\begin{array}{l}.25 \\
(2.1)\end{array}$ & $\begin{array}{l}.37 \\
(3.3)\end{array}$ & 33597 \\
\hline S95 & $\begin{array}{l}.48 \\
(2.3)\end{array}$ & $\begin{array}{l}11 \\
(42.4)\end{array}$ & $\begin{array}{l}.24 \\
(1.3)\end{array}$ & $\begin{array}{l}.56 \\
(3)\end{array}$ & $\begin{array}{l}.25 \\
(2.7)\end{array}$ & $\begin{array}{l}.06 \\
(.6)\end{array}$ & $\begin{array}{l}.68 \\
(2.8)\end{array}$ & 13514 \\
\hline
\end{tabular}

Standard deviation in parentheses. Monetary values in million $€ .^{\dagger}$ : head-count number of employees.

$\ddagger$ : KBC Intensity is defined as the the ratio of KBC stock over physical capital stock. 


\section{Appendix B. Production function results}

In Table B.1, we report the results from the GMM estimation of the production function $f(\cdot)$, at the 2-digit industry level. The column RTS reports the estimated returns to scale, as the sum of the output elasticities of labour and capital. In the manufacturing sector, the output elasticity of labour ranges from 0.4 in motor vehicle manufacturing (C29) to 0.91 in computer and electronics manufacturing (C26). The output elasticity of capital ranges from 0.14 in repair and installation of machinery to 0.53 in beverages (C11). The estimation procedure yields a very low (0.01), and statistically insignificant output elasticity of capital, for computer and electronics manufacturing (C26). In the services sector, the output elasticity of labour ranges from 0.36 in rental and leasing activities (N77) to 0.83 in computer programming activities (J62). The output elasticity of capital ranges from 0.05 in employment activities (N78) to 0.45 in land transport, including via pipelines (H49). The orders of magnitude of the output elasticities of labour and capital are generally consistent with those found in the literature. We find higher output elasticities of both inputs in the manufacturing sector compared to the services sector, resulting in higher returns to scale in the former compared to the latter. In the manufacturing sector, we find close to constant returns to scale across all industries, whereas in the services sector, especially in business services and administrative activities ( $\mathrm{M}$ and $\mathrm{N}$ ), the average returns to scale is 0.72 . 
Table B.1: Production function coefficients obtained from ACF procedure by 2-digit industry

\begin{tabular}{|c|c|c|c|c|c|c|c|c|c|}
\hline & $\beta_{L}$ & $\beta_{K}$ & RTS & $\mathrm{N}$ & & $\beta_{L}$ & $\beta_{K}$ & RTS & $\mathrm{N}$ \\
\hline $\mathrm{C} 10$ & $\begin{array}{l}.41^{* * *} \\
(.025)\end{array}$ & $\begin{array}{l}.47^{* * *} \\
(.026)\end{array}$ & .88 & 9482 & H51 & $\begin{array}{l}.63^{* * *} \\
(.081)\end{array}$ & $\begin{array}{l}.23^{* * *} \\
.046)\end{array}$ & .86 & 1482 \\
\hline C11 & $\begin{array}{l}.62^{* * *} \\
(.075)\end{array}$ & $\begin{array}{l}.53^{* * *} \\
(.066)\end{array}$ & 1.15 & 1297 & H52 & $\begin{array}{l}.53^{* * *} \\
(.012)\end{array}$ & $\begin{array}{l}.21^{* * *} \\
.01)\end{array}$ & .74 & 32179 \\
\hline $\mathrm{C} 13$ & $\begin{array}{l}.78^{* * *} \\
(.057)\end{array}$ & $\begin{array}{l}.18^{* *} \\
(.07)\end{array}$ & .96 & 1929 & H53 & $\begin{array}{l}.38^{* * *} \\
(.029)\end{array}$ & $\begin{array}{l}.34^{* * * *} \\
.045)\end{array}$ & .72 & 7820 \\
\hline C14 & $\begin{array}{l}.66^{* * *} \\
(.084)\end{array}$ & $\begin{array}{l}.31 \\
(.271)\end{array}$ & .97 & 1053 & J58 & $\begin{array}{l}.49^{* * *} \\
(.03)\end{array}$ & $\begin{array}{l}.41^{* * *} \\
(.026)\end{array}$ & .9 & 10931 \\
\hline $\mathrm{C} 15$ & $\begin{array}{l}.69^{* * *} \\
(.129)\end{array}$ & $\begin{array}{l}.41^{*} \\
(.169)\end{array}$ & 1.1 & 469 & J59 & $\begin{array}{l}.5 * * * \\
(.034)\end{array}$ & $\begin{array}{l}.23^{* * *} * \\
(.053)\end{array}$ & .73 & 5869 \\
\hline C16 & $\begin{array}{l}.76^{* * *} \\
(.069)\end{array}$ & $\begin{array}{l}.29^{* * *} \\
(.037)\end{array}$ & 1.05 & 1994 & $\mathrm{~J} 60$ & $\begin{array}{l}.48^{* *} \\
(.15)\end{array}$ & $\begin{array}{l}.31 \\
(.159)\end{array}$ & .79 & 838 \\
\hline $\mathrm{C} 17$ & $\begin{array}{l}.53^{* * *} \\
(.051)\end{array}$ & $\begin{array}{l}.33^{* * *} \\
(.03)\end{array}$ & .86 & 2257 & $\mathrm{~J} 61$ & $\begin{array}{l}.54^{* * *} \\
(.059)\end{array}$ & $\begin{array}{l}.33^{* * *} \\
(.068)\end{array}$ & .87 & 4212 \\
\hline C18 & $\begin{array}{l}.6^{* * *} \\
(.057)\end{array}$ & $\begin{array}{l}.25^{* * *} \\
(.043)\end{array}$ & .85 & 1635 & $\mathrm{~J} 62$ & $\begin{array}{l}.83^{* * *} \\
(.01)\end{array}$ & $\begin{array}{l}.18^{* * *} \\
(.01)\end{array}$ & 1.01 & 43097 \\
\hline $\mathrm{C} 20$ & $\begin{array}{l}.53^{* * *} \\
(.045)\end{array}$ & $\begin{array}{l}.33^{* * *} * \\
(.042)\end{array}$ & .86 & 4222 & J63 & $\begin{array}{l}.7 * * * \\
(.022)\end{array}$ & $\begin{array}{l}.2^{* * *} \\
(.019)\end{array}$ & .9 & 10904 \\
\hline $\mathrm{C} 21$ & $\begin{array}{l}.68^{* * *} \\
(.099)\end{array}$ & $\begin{array}{l}.35^{* *} \\
(.113)\end{array}$ & 1.03 & 913 & M69 & $\begin{array}{l}.79^{* * *} \\
(.009)\end{array}$ & $\begin{array}{l}.36^{* * *} \\
(.009)\end{array}$ & 1.15 & 81249 \\
\hline $\mathrm{C} 22$ & $\begin{array}{l}.62^{* * *} \\
(.034)\end{array}$ & $\begin{array}{l}.3^{* * *} \\
(.027)\end{array}$ & .92 & 4573 & M70 & $\begin{array}{l}.42^{* * *} \\
(.01)\end{array}$ & $\begin{array}{l}.14^{* * *} \\
(.007)\end{array}$ & .56 & 32538 \\
\hline $\mathrm{C} 23$ & $\begin{array}{l}.54^{* * *} \\
(.057)\end{array}$ & $\begin{array}{l}.3^{* * *} \\
(.037)\end{array}$ & .84 & 3763 & M71 & $\begin{array}{l}.49^{* * *} \\
(.01)\end{array}$ & $\begin{array}{l}.18^{* * *} \\
(.005)\end{array}$ & .67 & 59139 \\
\hline $\mathrm{C} 24$ & $\begin{array}{l}.68^{* * *} \\
(.037)\end{array}$ & $\begin{array}{l}.32^{* * *} \\
(.026)\end{array}$ & 1 & 3275 & M72 & $\begin{array}{l}.73^{* * *} \\
(.025)\end{array}$ & $\begin{array}{l}.16^{* * *} \\
(.021)\end{array}$ & .89 & 7208 \\
\hline $\mathrm{C} 25$ & $\begin{array}{l}.69^{* * *} \\
(.024)\end{array}$ & $\begin{array}{l}.26^{* * *} \\
(.015)\end{array}$ & .95 & 10430 & M73 & $\begin{array}{l}.44^{* * *} \\
(.017)\end{array}$ & $\begin{array}{l}.21 * * * \\
(.013)\end{array}$ & .65 & 26002 \\
\hline $\mathrm{C} 26$ & $\begin{array}{l}.91^{* * *} \\
(.038)\end{array}$ & $\begin{array}{l}.01 \\
(.037)\end{array}$ & .92 & 3664 & M74 & $\begin{array}{l}.55^{* * *} \\
(.015)\end{array}$ & $\begin{array}{l}.29 * * * \\
(.012)\end{array}$ & .84 & 17242 \\
\hline $\mathrm{C} 27$ & $\begin{array}{l}.75^{* * *} \\
(.029)\end{array}$ & $\begin{array}{l}.19^{* * *} \\
(.029)\end{array}$ & .94 & 5063 & M75 & $\begin{array}{l}.63^{* * *} \\
(.018)\end{array}$ & $\begin{array}{l}.31 * * * \\
(.017)\end{array}$ & .94 & 11214 \\
\hline $\mathrm{C} 28$ & $\begin{array}{l}.81^{* * *} \\
(.02)\end{array}$ & $\begin{array}{l}.2^{* * *} \\
(.016)\end{array}$ & 1.01 & 11500 & N77 & $\begin{array}{l}.36^{* * *} \\
(.022)\end{array}$ & $\begin{array}{l}.37 * * * \\
(.029)\end{array}$ & .73 & 18494 \\
\hline $\mathrm{C} 29$ & $\begin{array}{l}.4^{* *} \\
(.126)\end{array}$ & $\begin{array}{l}.43^{* * *} \\
(.091)\end{array}$ & .83 & 2974 & N78 & $\begin{array}{l}.68^{* * *} \\
(.01)\end{array}$ & $\begin{array}{l}.05 * * * \\
(.011)\end{array}$ & .73 & 16880 \\
\hline C30 & $\begin{array}{l}.85^{* * *} \\
(.08)\end{array}$ & $\begin{array}{l}.22 \\
(.144)\end{array}$ & 1.07 & 1000 & N79 & $\begin{array}{l}.59^{* * *} \\
(.018)\end{array}$ & $\begin{array}{l}.11^{* * *} * \\
(.012)\end{array}$ & .7 & 14240 \\
\hline C31 & $\begin{array}{l}.64^{* * *} \\
(.059)\end{array}$ & $\begin{array}{l}.28^{* * *} \\
(.037)\end{array}$ & .92 & 1875 & N80 & $\begin{array}{l}.45^{* * *} \\
(.024)\end{array}$ & $\begin{array}{l}.06 \\
(.032)\end{array}$ & .51 & 6746 \\
\hline C32 & $\begin{array}{l}.61^{* * *} \\
(.05)\end{array}$ & $\begin{array}{l}.25^{* * *} \\
(.052)\end{array}$ & .86 & 3042 & N81 & $\begin{array}{l}.46^{* * *} \\
(.01)\end{array}$ & $\begin{array}{l}.25^{* * *} \\
(.008)\end{array}$ & .71 & 48348 \\
\hline C33 & $\begin{array}{l}.83^{* * *} \\
(.046)\end{array}$ & $\begin{array}{l}.14^{* * *} \\
(.04)\end{array}$ & .97 & 3171 & N82 & $\begin{array}{l}.46^{* * *} \\
(.015)\end{array}$ & $\begin{array}{l}.21^{* * *} \\
(.015)\end{array}$ & .67 & 22407 \\
\hline H49 & $\begin{array}{l}.48^{* * *} \\
(.01)\end{array}$ & $\begin{array}{l}.45^{* * *} \\
(.012)\end{array}$ & .93 & 75957 & S95 & $\begin{array}{l}.78^{* * *} \\
.027)\end{array}$ & $\begin{array}{l}.23 * * * \\
.013)\end{array}$ & 1.01 & 8586 \\
\hline H50 & $\begin{array}{l}.66^{* * *} \\
(.035)\end{array}$ & $\begin{array}{l}.18^{* * *} \\
(.033)\end{array}$ & .84 & 7454 & & & & & \\
\hline
\end{tabular}

*** $\mathrm{p}<0.01, * * \mathrm{p}<0.05, * \mathrm{p}<0.1$. Standard errors in parentheses. RTS: Returns to Scale, calculated as the sum of $\beta_{K}$ and $\beta_{L}$ 


\section{Appendix C. Full results of robustness estimations}

Table C.1: Results of robustness tests, by 2-digit industry, linear law of motion of productivity

\begin{tabular}{|c|c|c|c|c|c|}
\hline \multirow[b]{2}{*}{ Industry } & \multicolumn{3}{|c|}{$\begin{array}{l}\text { Point estimates of the } \\
\text { law of motion of productivity }\end{array}$} & \multirow[b]{2}{*}{$R^{2}$} & \multirow[b]{2}{*}{$\mathrm{N}$} \\
\hline & Constant & $\beta_{c}$ & $\beta_{\omega}$ & & \\
\hline $\mathrm{C} 10$ & $\begin{array}{l}.48^{* * *} \\
(.02)\end{array}$ & $\begin{array}{c}.002^{* * *} \\
(.00)\end{array}$ & $\begin{array}{c}.914^{* * *} \\
(.00)\end{array}$ & .9 & 9482 \\
\hline C11 & $\begin{array}{l}.47^{* * *} \\
(.05)\end{array}$ & $\begin{array}{l}.00 \\
(.00)\end{array}$ & $\begin{array}{c}.917^{* * *} \\
(.01)\end{array}$ & .89 & 1297 \\
\hline $\mathrm{C} 13$ & $\begin{array}{c}.527^{* * *} \\
(.06)\end{array}$ & $\begin{array}{l}.001 \\
(.00)\end{array}$ & $\begin{array}{c}.937^{* * * *} \\
(.01)\end{array}$ & .89 & 1929 \\
\hline C14 & $\begin{array}{l}.376^{* * *} \\
(.07)\end{array}$ & $\begin{array}{c}.003^{* * *} \\
(.00)\end{array}$ & $\begin{array}{c}.959^{* * * *} \\
(.01)\end{array}$ & .95 & 1053 \\
\hline $\mathrm{C} 15$ & $\begin{array}{l}.315^{* * *} \\
(.08)\end{array}$ & $\begin{array}{l}.002 \\
(.00)\end{array}$ & $\begin{array}{c}.942^{* * *} \\
(.02)\end{array}$ & .89 & 469 \\
\hline $\mathrm{C} 16$ & $\begin{array}{c}.587^{* * * *} \\
(.06)\end{array}$ & $\begin{array}{r}-.001 \\
(.00)\end{array}$ & $\begin{array}{c}.921^{* * * *} \\
(.01)\end{array}$ & .86 & 1994 \\
\hline $\mathrm{C} 17$ & $\begin{array}{c}.455^{* * *} \\
(.04)\end{array}$ & $\begin{array}{c}.011 * * * \\
(.00)\end{array}$ & $\begin{array}{c}.921 * * * \\
(.01)\end{array}$ & .94 & 2257 \\
\hline $\mathrm{C} 18$ & $\begin{array}{l}.645^{* * *} \\
(.07)\end{array}$ & $\begin{array}{c}.002^{* * * *} \\
(.00)\end{array}$ & $\begin{array}{c}.907^{* * *} \\
(.01)\end{array}$ & .85 & 1635 \\
\hline $\mathrm{C} 20$ & $\begin{array}{c}.428^{* * * *} \\
(.03)\end{array}$ & $\begin{array}{c}.007^{* * * *} \\
(.00)\end{array}$ & $\begin{array}{c}.933^{* * *} \\
(.01)\end{array}$ & .92 & 4222 \\
\hline $\mathrm{C} 21$ & $\begin{array}{c}.735^{* * *} \\
(.09)\end{array}$ & $\begin{array}{c}.008^{* * *} \\
(.00)\end{array}$ & $\begin{array}{l}.89^{* * *} \\
(.01)\end{array}$ & .89 & 913 \\
\hline $\mathrm{C} 22$ & $\begin{array}{c}.708^{* * *} \\
(.04)\end{array}$ & $\begin{array}{c}.002^{* * *} \\
(.00)\end{array}$ & $\begin{array}{c}.903^{* * *} \\
(.01)\end{array}$ & .88 & 4573 \\
\hline $\mathrm{C} 23$ & $\begin{array}{l}.454^{* * *} \\
(.04)\end{array}$ & $\begin{array}{l}.001^{*} \\
(.00)\end{array}$ & $\begin{array}{c}.937^{* * *} \\
(.01)\end{array}$ & .88 & 3763 \\
\hline $\mathrm{C} 24$ & $\begin{array}{c}.977^{* * *} \\
(.05)\end{array}$ & $\begin{array}{c}.001^{* * * *} \\
(.00)\end{array}$ & $\begin{array}{c}.863^{* * * *} \\
(.01)\end{array}$ & .82 & 3275 \\
\hline $\mathrm{C} 25$ & $\begin{array}{c}.938^{* * *} \\
(.03)\end{array}$ & $\begin{array}{c}.001^{* * * *} \\
(.00)\end{array}$ & $\begin{array}{c}.88^{* * *} \\
(.00)\end{array}$ & .82 & 10430 \\
\hline $\mathrm{C} 26$ & $\begin{array}{l}1.037^{* * *} \\
(.07)\end{array}$ & $\begin{array}{c}.009^{* * *} \\
(.00)\end{array}$ & $\begin{array}{c}.896^{* * *} \\
(.01)\end{array}$ & .87 & 3664 \\
\hline $\mathrm{C} 27$ & $\begin{array}{c}.736^{* * *} \\
(.04)\end{array}$ & $\begin{array}{c}.005^{* * *} \\
(.00)\end{array}$ & $\begin{array}{c}.912^{* * *} \\
(.01)\end{array}$ & .9 & 5063 \\
\hline $\mathrm{C} 28$ & $\begin{array}{c}1.119^{* * *} \\
(.03)\end{array}$ & $\begin{array}{c}.005^{* * *} \\
(.00)\end{array}$ & $\begin{array}{c}.863^{* * *} \\
(.00)\end{array}$ & .81 & 11500 \\
\hline $\mathrm{C} 29$ & $\begin{array}{c}.547^{* * * *} \\
(.04)\end{array}$ & $\begin{array}{c}.005^{* * * *} \\
(.00)\end{array}$ & $\begin{array}{c}.903^{* * *} \\
(.01)\end{array}$ & .89 & 2974 \\
\hline $\mathrm{C} 30$ & $\begin{array}{c}.528^{* * *} \\
(.09)\end{array}$ & $\begin{array}{l}.001 \\
(.00)\end{array}$ & $\begin{array}{c}.929^{* * *} \\
(.01)\end{array}$ & .85 & 1000 \\
\hline C31 & $\begin{array}{c}.996^{* * * *} \\
(.08)\end{array}$ & $\begin{array}{c}.015^{* * *} \\
(.00)\end{array}$ & $\begin{array}{c}.852^{* * *} \\
(.01)\end{array}$ & .85 & 1875 \\
\hline C32 & $\begin{array}{c}.475^{* * * *} \\
(.04)\end{array}$ & $\begin{array}{c}.001^{* * * *} \\
(.00)\end{array}$ & $\begin{array}{c}.931^{* * *} \\
(.01)\end{array}$ & .9 & 3042 \\
\hline C33 & $\begin{array}{l}.8^{* * * *} \\
(.06)\end{array}$ & $\begin{array}{c}.001^{* * *} \\
(.00)\end{array}$ & $\begin{array}{c}.909^{* * * *} \\
(.01)\end{array}$ & .85 & 3171 \\
\hline
\end{tabular}

$* * * \mathrm{p}<0.01,{ }^{* *} \mathrm{p}<0.05, * \mathrm{p}<0.1$. Standard errors in parentheses. 
Table C.2: Results of robustness tests, by 2-digit industry, linear law of motion of productivity

\begin{tabular}{|c|c|c|c|c|c|}
\hline \multirow[b]{2}{*}{ Industry } & \multicolumn{3}{|c|}{$\begin{array}{c}\text { Point estimates of the } \\
\text { law of motion of productivity }\end{array}$} & \multirow[b]{2}{*}{$R^{2}$} & \multirow[b]{2}{*}{$\mathrm{N}$} \\
\hline & Constant & $\beta_{c}$ & $\beta_{\omega}$ & & \\
\hline H49 & $\begin{array}{c}.739^{* * *} \\
(.01)\end{array}$ & $\begin{array}{c}.008^{* * *} \\
(.00)\end{array}$ & $\begin{array}{c}.842^{* * *} \\
(.00)\end{array}$ & .8 & 75957 \\
\hline H50 & $\begin{array}{c}1.099^{* * *} \\
(.04)\end{array}$ & $\begin{array}{c}.009^{* * *} \\
(.00)\end{array}$ & $\begin{array}{c}.846^{* * *} \\
(.01)\end{array}$ & .75 & 7454 \\
\hline H51 & $\begin{array}{c}2.057^{* * *} \\
(.12)\end{array}$ & $\begin{array}{c}.023^{* * *} \\
(.00)\end{array}$ & $\begin{array}{c}.735^{* * *} \\
(.01)\end{array}$ & .77 & 1482 \\
\hline H52 & $\begin{array}{c}1.23^{* * * *} \\
(.02)\end{array}$ & $\begin{array}{c}.012^{* * * *} \\
(.00)\end{array}$ & $\begin{array}{c}.835^{* * * *} \\
(.00)\end{array}$ & .82 & 32179 \\
\hline H53 & $\begin{array}{c}.553^{* * * *} \\
(.03)\end{array}$ & $\begin{array}{c}.007^{* * * *} \\
(.00)\end{array}$ & $\begin{array}{c}.917^{* * * *} \\
(.00)\end{array}$ & .88 & 7820 \\
\hline J58 & $\begin{array}{c}.368^{* * * *} \\
(.02)\end{array}$ & $\begin{array}{c}.003^{* * *} \\
(.00)\end{array}$ & $\begin{array}{c}.929 * * * \\
(.00)\end{array}$ & .89 & 10931 \\
\hline J59 & $\begin{array}{c}.624 * * * \\
(.03)\end{array}$ & $\begin{array}{c}.012 * * * \\
(.00)\end{array}$ & $\begin{array}{c}.908^{* * *} \\
(.01)\end{array}$ & .89 & 5869 \\
\hline J60 & $\begin{array}{c}.445^{* * * *} \\
(.08)\end{array}$ & $\begin{array}{c}.02^{* * *} \\
(.00)\end{array}$ & $\begin{array}{c}.892^{* * * *} \\
(.01)\end{array}$ & .88 & 838 \\
\hline J61 & $\begin{array}{c}.625^{* * *} \\
(.05)\end{array}$ & $\begin{array}{c}.015^{* * * *} \\
(.00)\end{array}$ & $\begin{array}{c}.877^{* * * *} \\
(.01)\end{array}$ & .81 & 4212 \\
\hline $\mathrm{J} 62$ & $\begin{array}{c}1.713^{* * *} \\
(.02)\end{array}$ & $\begin{array}{c}.028^{* * *} \\
(.00)\end{array}$ & $\begin{array}{c}.778^{* * *} \\
(.00)\end{array}$ & .71 & 43097 \\
\hline J63 & $\begin{array}{c}1.12^{* * * *} \\
(.03)\end{array}$ & $\begin{array}{c}.005^{* * *} \\
(.00)\end{array}$ & $\begin{array}{c}.852^{* * * *} \\
(.00)\end{array}$ & .78 & 10904 \\
\hline M69 & $\begin{array}{c}1.117^{* * *} \\
(.01)\end{array}$ & $\begin{array}{c}.006^{* * * *} \\
(.00)\end{array}$ & $\begin{array}{c}.824^{* * * *} \\
(.00)\end{array}$ & .73 & 81249 \\
\hline M70 & $\begin{array}{c}1.025^{* * *} \\
(.02)\end{array}$ & $\begin{array}{c}.027^{* * * *} \\
(.00)\end{array}$ & $\begin{array}{c}.843^{* * * *} \\
(.00)\end{array}$ & .83 & 32538 \\
\hline M71 & $\begin{array}{c}1.619^{* * *} \\
(.02)\end{array}$ & $\begin{array}{c}.076^{* * * *} \\
(.00)\end{array}$ & $\begin{array}{c}.717^{* * * *} \\
(.00)\end{array}$ & .89 & 59139 \\
\hline M72 & $\begin{array}{c}1.162^{* * *} \\
(.05)\end{array}$ & $\begin{array}{c}.017^{* * * *} \\
(.00)\end{array}$ & $\begin{array}{c}.845^{* * * *} \\
(.01)\end{array}$ & .8 & 7208 \\
\hline M73 & $\begin{array}{c}.886^{* * * *} \\
(.02)\end{array}$ & $\begin{array}{c}.014^{* * * *} \\
(.00)\end{array}$ & $\begin{array}{c}.873^{* * * *} \\
(.00)\end{array}$ & .86 & 26002 \\
\hline M74 & $\begin{array}{c}1.161 * * * \\
(.03)\end{array}$ & $\begin{array}{c}.012 * * * \\
(.00)\end{array}$ & $\begin{array}{c}.835^{* * * *} \\
(.00)\end{array}$ & .85 & 17242 \\
\hline M75 & $\begin{array}{c}1.559 * * * \\
(.04)\end{array}$ & $\begin{array}{c}.006^{* * *} \\
(.00)\end{array}$ & $\begin{array}{c}.781 * * * \\
(.01)\end{array}$ & .62 & 11214 \\
\hline N77 & $\begin{array}{c}.567^{* * *} \\
(.02)\end{array}$ & $\begin{array}{c}.014^{* * *} \\
(.00)\end{array}$ & $\begin{array}{c}.886^{* * *} \\
(.00)\end{array}$ & .87 & 18494 \\
\hline N78 & $\begin{array}{c}2.597^{* * *} \\
(.05)\end{array}$ & $\begin{array}{c}.01 * * * \\
(.00)\end{array}$ & $\begin{array}{c}.721^{* * *} \\
(.00)\end{array}$ & .59 & 16880 \\
\hline N79 & $\begin{array}{c}1.543^{* * *} \\
(.04)\end{array}$ & $\begin{array}{c}.021^{* * *} \\
(.00)\end{array}$ & $\begin{array}{c}.81^{* * *} \\
(.00)\end{array}$ & .8 & 14240 \\
\hline N80 & $\begin{array}{c}1.035^{* * *} \\
(.05)\end{array}$ & $\begin{array}{c}.019^{* * *} \\
(.00)\end{array}$ & $\begin{array}{c}.849^{* * *} \\
(.01)\end{array}$ & .82 & 6746 \\
\hline N81 & $\begin{array}{c}1.13^{* * *} \\
(.02)\end{array}$ & $\begin{array}{c}.005^{* * *} \\
(.00)\end{array}$ & $\begin{array}{c}.849^{* * *} \\
(.00)\end{array}$ & .77 & 48348 \\
\hline N82 & $\begin{array}{c}.776^{* * *} \\
(.02)\end{array}$ & $\begin{array}{c}.01^{* * *} \\
(.00)\end{array}$ & $\begin{array}{c}.893^{* * *} \\
(.00)\end{array}$ & .86 & 22407 \\
\hline S95 & $\begin{array}{c}1.358^{* * *} \\
(.05)\end{array}$ & $\begin{array}{c}.003^{* * *} \\
(.00)\end{array}$ & $\begin{array}{c}.81^{* 2 *} \\
(.01)\end{array}$ & .68 & 8586 \\
\hline
\end{tabular}

$* * * \mathrm{p}<0.01, * * \mathrm{p}<0.05, * \mathrm{p}<0.1$. Standard errors in parentheses. 
Table C.3: Results of robustness tests, by 2-digit industry, KBC without OC

\begin{tabular}{|c|c|c|c|c|c|c|c|}
\hline \multirow[b]{2}{*}{ Industry } & \multicolumn{6}{|c|}{ Point estimates of the law of motion of productivity } & \multirow[b]{2}{*}{$\mathrm{N}$} \\
\hline & Constant & $\beta_{c, n o O C}$ & $\beta_{c^{2}, \text { noOC }}$ & $\beta_{\omega, \text { noOC }}$ & $\beta_{\omega^{2}, \text { noOC }}$ & $\beta_{c \omega, n o O C}$ & \\
\hline C10 & $\begin{array}{c}1.454^{* * *} \\
(.16)\end{array}$ & $\begin{array}{c}.016^{* * *} \\
(.00)\end{array}$ & $\begin{array}{l}0^{* * *} \\
(.00)\end{array}$ & $\begin{array}{c}.545^{* * *} \\
(.06)\end{array}$ & $\begin{array}{c}.035^{* * *} * \\
(.01)\end{array}$ & $\begin{array}{c}-.003^{* * *} \\
(.00)\end{array}$ & 9482 \\
\hline $\mathrm{C} 11$ & $\begin{array}{c}2.945^{* * *} \\
(.5)\end{array}$ & $\begin{array}{c}.024^{* *} \\
(.01)\end{array}$ & $\begin{array}{l}.00 \\
. .00)\end{array}$ & $\begin{array}{l}.166 \\
(.14)\end{array}$ & $\begin{array}{c}.059^{* * *} \\
(.01)\end{array}$ & $\begin{array}{c}-.003^{* *} \\
(.00)\end{array}$ & 1297 \\
\hline $\mathrm{C} 13$ & $\begin{array}{c}-2.242^{* * *} \\
(.71)\end{array}$ & $\begin{array}{c}-.03^{* *} \\
(.01)\end{array}$ & $\begin{array}{l}.00 \\
(.00)\end{array}$ & $\begin{array}{c}1.675^{* * * *} \\
(.19)\end{array}$ & $\begin{array}{c}-.049^{* * *} \\
(.01)\end{array}$ & $\begin{array}{c}.004^{* *} \\
(.00)\end{array}$ & 1929 \\
\hline $\mathrm{C} 14$ & $\begin{array}{c}.818^{* * *} \\
(.25)\end{array}$ & $\begin{array}{c}.023^{* * * *} \\
(.01)\end{array}$ & $\begin{array}{c}0^{*} \\
(.00)\end{array}$ & $\begin{array}{c}.675^{* * *} \\
(.11)\end{array}$ & $\begin{array}{c}.032^{* * * *} \\
(.01)\end{array}$ & $\begin{array}{c}-.004^{*} \\
(.00)\end{array}$ & 1053 \\
\hline $\mathrm{C} 15$ & $\begin{array}{c}1.57^{* * * *} \\
(.38)\end{array}$ & $\begin{array}{l}.018 \\
(.02)\end{array}$ & $\begin{array}{l}.00 \\
(.00)\end{array}$ & $\begin{array}{c}.359^{* *} \\
(.17)\end{array}$ & $\begin{array}{c}.065^{* * *} \\
(.02)\end{array}$ & $\begin{array}{r}-.003 \\
(.00)\end{array}$ & 469 \\
\hline C16 & $\begin{array}{c}-1.622^{*} \\
(.93)\end{array}$ & $\begin{array}{l}.003 \\
(.01)\end{array}$ & $\begin{array}{l}.00 \\
(.00)\end{array}$ & $\begin{array}{c}1.54^{* * * *} \\
(.26)\end{array}$ & $\begin{array}{c}-.043^{* *} \\
(.02)\end{array}$ & $\begin{array}{l}.00 \\
(.00)\end{array}$ & 1994 \\
\hline $\mathrm{C} 17$ & $\begin{array}{c}1.379^{* * * *} \\
(.46)\end{array}$ & $\begin{array}{c}.025^{* * * *} \\
(.01)\end{array}$ & $\begin{array}{l}.00 \\
(.00)\end{array}$ & $\begin{array}{c}.605^{* * * *} \\
(.15)\end{array}$ & $\begin{array}{c}.028 * * \\
(.01)\end{array}$ & $\begin{array}{c}-.004^{* *} \\
(.00)\end{array}$ & 2257 \\
\hline C18 & $\begin{array}{l}.267 \\
(.87)\end{array}$ & $\begin{array}{c}.047^{* * * *} \\
(.01)\end{array}$ & $\begin{array}{c}.001^{* * *} \\
(.00)\end{array}$ & $\begin{array}{c}.974 * * * \\
(.24)\end{array}$ & $\begin{array}{r}-.002 \\
(.02)\end{array}$ & $\begin{array}{c}-.007^{* * * *} \\
(.00)\end{array}$ & 1635 \\
\hline $\mathrm{C} 20$ & $\begin{array}{c}.936^{* * * *} \\
(.32)\end{array}$ & $\begin{array}{c}.017^{* * * *} \\
(.01)\end{array}$ & $\begin{array}{l}0^{* * *} \\
(.00)\end{array}$ & $\begin{array}{c}.759^{* * *} \\
(.1)\end{array}$ & $\begin{array}{c}.015^{* *} \\
(.01)\end{array}$ & $\begin{array}{c}-.003^{* * *} \\
(.00)\end{array}$ & 4222 \\
\hline $\mathrm{C} 21$ & $\begin{array}{c}4.598^{* * *} \\
(.68)\end{array}$ & $\begin{array}{l}.053^{* *} \\
(.02)\end{array}$ & $\begin{array}{c}0^{*} \\
(.00)\end{array}$ & $\begin{array}{c}-.402^{*} \\
(.21)\end{array}$ & $\begin{array}{c}.107^{* * *} \\
(.02)\end{array}$ & $\begin{array}{c}-.009^{* * *} \\
(.00)\end{array}$ & 913 \\
\hline $\mathrm{C} 22$ & $\begin{array}{l}.823 \\
(.54)\end{array}$ & $\begin{array}{l}.014^{*} \\
(.01)\end{array}$ & $\begin{array}{l}0^{* * *} \\
(.00)\end{array}$ & $\begin{array}{c}.862^{* * * *} \\
(.15)\end{array}$ & $\begin{array}{l}.004 \\
(.01)\end{array}$ & $\begin{array}{c}-.002^{*} \\
(.00)\end{array}$ & 4573 \\
\hline $\mathrm{C} 23$ & $\begin{array}{l}.296 \\
(.55)\end{array}$ & $\begin{array}{r}-.009 \\
(.01)\end{array}$ & $\begin{array}{l}0^{* * *} \\
(.00)\end{array}$ & $\begin{array}{c}1.002^{* * *} \\
(.15)\end{array}$ & $\begin{array}{l}-.006 \\
(.01)\end{array}$ & $\begin{array}{l}.001 \\
(.00)\end{array}$ & 3763 \\
\hline $\mathrm{C} 24$ & $\begin{array}{c}2.349 * * * \\
(.82)\end{array}$ & $\begin{array}{l}.008 \\
(.01)\end{array}$ & $\begin{array}{l}.00 \\
(.00)\end{array}$ & $\begin{array}{c}.473^{* *} \\
(.23)\end{array}$ & $\begin{array}{l}.028^{*} \\
(.02)\end{array}$ & $\begin{array}{r}-.001 \\
(.00)\end{array}$ & 3275 \\
\hline $\mathrm{C} 25$ & $\begin{array}{r}-.287 \\
(.56)\end{array}$ & $\begin{array}{c}-.021^{* * *} * \\
(.01)\end{array}$ & $\begin{array}{c}0^{*} \\
(.00)\end{array}$ & $\begin{array}{c}1.222^{* * *} \\
(.15)\end{array}$ & $\begin{array}{c}-.023^{* *} \\
(.01)\end{array}$ & $\begin{array}{c}.003^{* * *} \\
(.00)\end{array}$ & 10430 \\
\hline $\mathrm{C} 26$ & $\begin{array}{c}6.274^{* * * *} \\
(1.58)\end{array}$ & $\begin{array}{c}.077^{* * * *} \\
(.02)\end{array}$ & $\begin{array}{c}.001^{* * * *} \\
(.00)\end{array}$ & $\begin{array}{l}-.16 \\
(.31)\end{array}$ & $\begin{array}{c}.054^{* * *} \\
(.02)\end{array}$ & $\begin{array}{c}-.008^{* * * *} \\
(.00)\end{array}$ & 3664 \\
\hline $\mathrm{C} 27$ & $\begin{array}{l}.619 \\
(.57)\end{array}$ & $\begin{array}{c}.046^{* * * *} \\
(.01)\end{array}$ & $\begin{array}{l}0^{* * *} \\
(.00)\end{array}$ & $\begin{array}{c}.904^{* * * *} \\
(.14)\end{array}$ & $\begin{array}{l}.003 \\
(.01)\end{array}$ & $\begin{array}{c}-.005^{* * *} \\
(.00)\end{array}$ & 5063 \\
\hline $\mathrm{C} 28$ & $\begin{array}{l}-.489 \\
(.63)\end{array}$ & $\begin{array}{c}-.02^{* * *} \\
(.01)\end{array}$ & $\begin{array}{l}0^{* * *} \\
(.00)\end{array}$ & $\begin{array}{c}1.276^{* * * *} \\
(.15)\end{array}$ & $\begin{array}{c}-.025^{* * *} \\
(.01)\end{array}$ & $\begin{array}{c}.002^{* * * *} \\
(.00)\end{array}$ & 11500 \\
\hline $\mathrm{C} 29$ & $\begin{array}{c}2.128^{* * * *} \\
(.31)\end{array}$ & $\begin{array}{c}.042^{* * * *} \\
(.01)\end{array}$ & $\begin{array}{c}.001 * * * \\
(.00)\end{array}$ & $\begin{array}{c}.284^{* *} \\
(.12)\end{array}$ & $\begin{array}{c}.062^{* * * *} \\
(.01)\end{array}$ & $\begin{array}{c}-.008^{* * * *} \\
(.00)\end{array}$ & 2974 \\
\hline C30 & $\begin{array}{c}.503 \\
(1.55)\end{array}$ & $\begin{array}{l}.005 \\
(.02)\end{array}$ & $\begin{array}{l}.00 \\
(.00)\end{array}$ & $\begin{array}{l}.921^{* *} \\
(.41)\end{array}$ & $\begin{array}{l}.001 \\
(.03)\end{array}$ & $\begin{array}{l}-.001 \\
(.00)\end{array}$ & 1000 \\
\hline $\mathrm{C} 31$ & $\begin{array}{l}.397 \\
(1.9)\end{array}$ & $\begin{array}{l}.001 \\
(.02)\end{array}$ & $\begin{array}{c}0^{*} \\
(.00)\end{array}$ & $\begin{array}{c}1.036^{* *} \\
(.51)\end{array}$ & $\begin{array}{l}-.012 \\
(.03)\end{array}$ & $\begin{array}{l}.00 \\
(.00)\end{array}$ & 1875 \\
\hline C32 & $\begin{array}{c}2.419^{* * * *} \\
(.61)\end{array}$ & $\begin{array}{l}.007 \\
(.01)\end{array}$ & $\begin{array}{l}0^{* * * *} \\
(.00)\end{array}$ & $\begin{array}{l}.422^{* *} \\
(.17)\end{array}$ & $\begin{array}{c}.034^{* * *} \\
(.01)\end{array}$ & $\begin{array}{l}-.001 \\
(.00)\end{array}$ & 3042 \\
\hline C33 & $\begin{array}{l}.533 \\
(.95)\end{array}$ & $\begin{array}{l}-.005 \\
(.01)\end{array}$ & $\begin{array}{l}.00 \\
(.00)\end{array}$ & $\begin{array}{c}.981 * * * \\
(.21)\end{array}$ & $\begin{array}{l}-.004 \\
(.01)\end{array}$ & $\begin{array}{l}.001 \\
(.00)\end{array}$ & 3171 \\
\hline
\end{tabular}

*** $\mathrm{p}<0.01,{ }^{* *} \mathrm{p}<0.05,{ }^{*} \mathrm{p}<0.1$. Standard errors in parentheses. 
Table C.4: Results of robustness tests, by 2-digit industry, KBC without OC

\begin{tabular}{|c|c|c|c|c|c|c|c|}
\hline \multirow[b]{2}{*}{ Industry } & \multicolumn{6}{|c|}{ Point estimates of the law of motion of productivity } & \multirow[b]{2}{*}{$\mathrm{N}$} \\
\hline & Constant & $\beta_{c, n o O C}$ & $\beta_{c^{2}, n o O C}$ & $\beta_{\omega, n \circ O C}$ & $\beta_{\omega^{2}, n o O C}$ & $\beta_{c \omega, n o O C}$ & \\
\hline $\mathrm{H} 49$ & $\begin{array}{c}.708^{* * *} \\
(.03)\end{array}$ & $\begin{array}{l}.003 \\
(.00)\end{array}$ & $\begin{array}{l}0 * * * \\
(.00)\end{array}$ & $\begin{array}{c}.817^{* * *} \\
(.01)\end{array}$ & $\begin{array}{c}.003^{* *} \\
(.00)\end{array}$ & $\begin{array}{c}.001^{* * *} \\
(.00)\end{array}$ & 75957 \\
\hline $\mathrm{H} 50$ & $\begin{array}{c}-2.325^{* * *} \\
(.3)\end{array}$ & $\begin{array}{l}.015 \\
(.01)\end{array}$ & $\begin{array}{c}.001^{* *} \\
(.00)\end{array}$ & $\begin{array}{c}1.665^{* * * *} \\
(.07)\end{array}$ & $\begin{array}{c}-.046^{* * *} \\
(.00)\end{array}$ & $\begin{array}{r}-.002 \\
(.00)\end{array}$ & 7454 \\
\hline H51 & $\begin{array}{c}-2.919^{* * *} \\
(.24)\end{array}$ & $\begin{array}{l}.005 \\
(.02)\end{array}$ & $\begin{array}{c}.001^{* *} \\
(.00)\end{array}$ & $\begin{array}{c}1.884^{* * * *} \\
(.05)\end{array}$ & $\begin{array}{c}-.065^{* * *} \\
(.00)\end{array}$ & $\begin{array}{l}.00 \\
(.00)\end{array}$ & 1482 \\
\hline $\mathrm{H} 52$ & $\begin{array}{l}.262 \\
(.19)\end{array}$ & $\begin{array}{c}.014^{* * * *} \\
(.00)\end{array}$ & $\begin{array}{l}0^{* * *} \\
(.00)\end{array}$ & $\begin{array}{c}1.069^{* * *} * \\
(.05)\end{array}$ & $\begin{array}{c}-.013^{* * *} \\
(.00)\end{array}$ & $\begin{array}{c}-.001^{*} \\
(.00)\end{array}$ & 32179 \\
\hline H53 & $\begin{array}{c}1.08^{* * *} * \\
(.19)\end{array}$ & $\begin{array}{c}-.005 \\
(.01)\end{array}$ & $\begin{array}{l}.00 \\
(.00)\end{array}$ & $\begin{array}{c}.787^{* * *} \\
(.06)\end{array}$ & $\begin{array}{l}.007^{*} \\
(.00)\end{array}$ & $\begin{array}{c}.002^{* *} \\
(.00)\end{array}$ & 7820 \\
\hline J58 & $\begin{array}{c}-.256 * * * \\
(.08)\end{array}$ & $\begin{array}{c}.015^{* * *} \\
(.00)\end{array}$ & $\begin{array}{c}.001^{* * *} \\
(.00)\end{array}$ & $\begin{array}{c}1.14^{* * *} * \\
(.03)\end{array}$ & $\begin{array}{c}-.016^{* * *} \\
(.00)\end{array}$ & $\begin{array}{c}-.004^{* * *} * \\
(.00)\end{array}$ & 10931 \\
\hline J59 & $\begin{array}{l}.032 \\
(.21)\end{array}$ & $\begin{array}{l}-.01 \\
(.01)\end{array}$ & $\begin{array}{l}.00 \\
(.00)\end{array}$ & $\begin{array}{c}1.095^{* * *} * \\
(.06)\end{array}$ & $\begin{array}{c}-.014^{* * *} \\
(.00)\end{array}$ & $\begin{array}{c}.002^{* *} \\
(.00)\end{array}$ & 5869 \\
\hline $\mathrm{J} 60$ & $\begin{array}{c}.559 * * \\
(.27)\end{array}$ & $\begin{array}{l}.01 \\
(.01)\end{array}$ & $\begin{array}{c}.001^{* *} \\
(.00)\end{array}$ & $\begin{array}{c}.87^{* * *} * \\
(.08)\end{array}$ & $\begin{array}{l}.004 \\
(.01)\end{array}$ & $\begin{array}{c}-.004 \\
(.00)\end{array}$ & 838 \\
\hline $\mathrm{J} 61$ & $\begin{array}{c}1.473^{* * *} \\
(.19)\end{array}$ & $\begin{array}{l}.008 \\
(.01)\end{array}$ & $\begin{array}{c}.001^{* * *} \\
(.00)\end{array}$ & $\begin{array}{c}.591^{* * *} \\
(.06)\end{array}$ & $\begin{array}{c}.025 * * * \\
(.01)\end{array}$ & $\begin{array}{l}-.002 \\
(.00)\end{array}$ & 4212 \\
\hline $\mathrm{J} 62$ & $\begin{array}{c}1.853^{* * *} * \\
(.22)\end{array}$ & $\begin{array}{l}.002 \\
(.00)\end{array}$ & $\begin{array}{c}0^{*} \\
(.00)\end{array}$ & $\begin{array}{c}.767^{* * *} \\
(.05)\end{array}$ & $\begin{array}{l}.001 \\
(.00)\end{array}$ & $\begin{array}{l}.00 \\
(.00)\end{array}$ & 43097 \\
\hline $\mathrm{J} 63$ & $\begin{array}{c}-1.329^{* * *} \\
(.21)\end{array}$ & $\begin{array}{c}-.05^{* * *} \\
(.01)\end{array}$ & $\begin{array}{c}.001^{* * *} \\
(.00)\end{array}$ & $\begin{array}{c}1.541^{* * * *} \\
(.05)\end{array}$ & $\begin{array}{c}-.046^{* * *} \\
(.00)\end{array}$ & $\begin{array}{c}.004^{* * *} \\
(.00)\end{array}$ & 10904 \\
\hline M69 & $\begin{array}{c}.65^{* * *} \\
(.1)\end{array}$ & $\begin{array}{l}.005 \\
(.00)\end{array}$ & $\begin{array}{l}0 * * * \\
(.00)\end{array}$ & $\begin{array}{c}.968^{* * *} \\
(.03)\end{array}$ & $\begin{array}{c}-.011^{* * *} \\
(.00)\end{array}$ & $\begin{array}{l}.001 \\
(.00)\end{array}$ & 81249 \\
\hline M70 & $\begin{array}{c}2.957^{* * *} * \\
(.16)\end{array}$ & $\begin{array}{c}.104^{* * *} \\
(.01)\end{array}$ & $\begin{array}{c}.008^{* * *} \\
(.00)\end{array}$ & $\begin{array}{c}.444 * * * \\
(.03)\end{array}$ & $\begin{array}{c}.025^{* * *} \\
(.00)\end{array}$ & $\begin{array}{c}-.02^{* * *} \\
(.00)\end{array}$ & 32538 \\
\hline M71 & $\begin{array}{c}13.23^{* * *} \\
(.16)\end{array}$ & $\begin{array}{c}.216^{* * * *} \\
(.02)\end{array}$ & $\begin{array}{c}.022^{* * *} \\
(.00)\end{array}$ & $\begin{array}{c}-1.72^{* * *} \\
(.05)\end{array}$ & $\begin{array}{c}.159 * * * \\
(.00)\end{array}$ & $\begin{array}{c}-.066^{* * *} \\
(.00)\end{array}$ & 59139 \\
\hline M72 & $\begin{array}{c}2.092^{* * *} \\
(.2)\end{array}$ & $\begin{array}{c}.035^{* * *} \\
(.01)\end{array}$ & $\begin{array}{c}.00 \\
(.00)\end{array}$ & $\begin{array}{c}.425^{* * *} \\
(.06)\end{array}$ & $\begin{array}{c}.038 * * * \\
(.01)\end{array}$ & $\begin{array}{c}-.005^{* * *} \\
(.00)\end{array}$ & 7208 \\
\hline M73 & $\begin{array}{r}-.037 \\
(.19)\end{array}$ & $\begin{array}{c}-.012^{* *} \\
(.01)\end{array}$ & $\begin{array}{c}.002^{* * * *} \\
(.00)\end{array}$ & $\begin{array}{c}1.122^{* * *} \\
(.04)\end{array}$ & $\begin{array}{c}-.015^{* * *} \\
(.00)\end{array}$ & $\begin{array}{r}-.001 \\
(.00)\end{array}$ & 26002 \\
\hline M74 & $\begin{array}{c}2.353^{* * *} * \\
(.23)\end{array}$ & $\begin{array}{c}.036^{* * *} \\
(.01)\end{array}$ & $\begin{array}{l}0^{* * *} \\
(.00)\end{array}$ & $\begin{array}{c}.488^{* * *} \\
(.06)\end{array}$ & $\begin{array}{c}.025^{* * *} \\
(.00)\end{array}$ & $\begin{array}{c}-.004^{* * *} \\
(.00)\end{array}$ & 17242 \\
\hline M75 & $\begin{array}{c}-4.301 * * * \\
(.57)\end{array}$ & $\begin{array}{c}-.071^{* * *} * \\
(.01)\end{array}$ & $\begin{array}{l}.00 \\
(.00)\end{array}$ & $\begin{array}{c}2.407^{* * *} * \\
(.16)\end{array}$ & $\begin{array}{c}-.113^{* * *} \\
(.01)\end{array}$ & $\begin{array}{c}.011^{* * *} \\
(.00)\end{array}$ & 11214 \\
\hline N77 & $\begin{array}{c}.996^{* * *} \\
(.07)\end{array}$ & $\begin{array}{c}.015^{* * *} \\
(.00)\end{array}$ & $\begin{array}{c}.001^{* * *} \\
(.00)\end{array}$ & $\begin{array}{c}.74^{* * *} \\
(.02)\end{array}$ & $\begin{array}{c}.013^{* * *} \\
(.00)\end{array}$ & $\begin{array}{c}-.003^{* * *} \\
(.00)\end{array}$ & 18494 \\
\hline N78 & $\begin{array}{c}-.587^{*} \\
(.35)\end{array}$ & $\begin{array}{r}-.015 \\
(.01)\end{array}$ & $\begin{array}{c}.001^{* * *} * \\
(.00)\end{array}$ & $\begin{array}{c}1.413^{* * * *} \\
(.07)\end{array}$ & $\begin{array}{c}-.037 * * * \\
(.00)\end{array}$ & $\begin{array}{l}.001 \\
(.00)\end{array}$ & 16880 \\
\hline N79 & $\begin{array}{c}-5.029^{* * *} \\
(.4)\end{array}$ & $\begin{array}{c}-.035^{* * *} * \\
(.01)\end{array}$ & $\begin{array}{c}.00 \\
(.00)\end{array}$ & $\begin{array}{c}2.32^{* * *} \\
(.09)\end{array}$ & $\begin{array}{c}-.086^{* * *} \\
(.01)\end{array}$ & $\begin{array}{c}.005^{* * *} \\
(.00)\end{array}$ & 14240 \\
\hline N80 & $\begin{array}{c}1.496^{* * *} \\
(.28)\end{array}$ & $\begin{array}{r}-.004 \\
(.01)\end{array}$ & $\begin{array}{l}.00 \\
(.00)\end{array}$ & $\begin{array}{c}.728^{* * *} \\
(.08)\end{array}$ & $\begin{array}{l}.008 \\
(.01)\end{array}$ & $\begin{array}{l}.001 \\
(.00)\end{array}$ & 6746 \\
\hline N81 & $\begin{array}{c}-.435^{* * *} \\
(.17)\end{array}$ & $\begin{array}{c}-.019^{* * * *} \\
(.00)\end{array}$ & $\begin{array}{l}0 * * \\
(.00)\end{array}$ & $\begin{array}{c}1.27^{* * *} * \\
(.04)\end{array}$ & $\begin{array}{c}-.028^{* * *} \\
(.00)\end{array}$ & $\begin{array}{c}.003^{* * *} \\
(.00)\end{array}$ & 48348 \\
\hline N82 & $\begin{array}{c}1.525^{* * *} \\
(.18)\end{array}$ & $\begin{array}{c}.025^{* * *} \\
(.00)\end{array}$ & $\begin{array}{l}0 * * * \\
(.00)\end{array}$ & $\begin{array}{c}.704^{* * *} \\
(.04)\end{array}$ & $\begin{array}{c}.012^{* * *} \\
(.00)\end{array}$ & $\begin{array}{c}-.002^{* * *} \\
(.00)\end{array}$ & 22407 \\
\hline S95 & $\begin{array}{c}6.651^{* * *} * \\
(.56)\end{array}$ & $\begin{array}{c}.069^{* * *} \\
(.01)\end{array}$ & $\begin{array}{c}0^{*} \\
(.00)\end{array}$ & $\begin{array}{c}-.509^{* * *} \\
(.14)\end{array}$ & $\begin{array}{c}.083^{* * *} \\
(.01)\end{array}$ & $\begin{array}{c}-.008^{* * *} * \\
(.00)\end{array}$ & 8586 \\
\hline
\end{tabular}

*** $\mathrm{p}<0.01, * * \mathrm{p}<0.05, * \mathrm{p}<0.1$. Standard errors in parentheses. 
Table C.5: Results of robustness tests, by 2-digit industry, KBC Investment

\begin{tabular}{|c|c|c|c|c|c|c|c|}
\hline Industry & Constant & $\begin{array}{c}\text { Point estir } \\
\beta_{c, \text { Invest }}\end{array}$ & $\begin{array}{l}\text { tes of the la } \\
\beta_{c^{2}, \text { Invest }}\end{array}$ & $\begin{array}{l}\text { of motion } \\
\beta_{\omega, \text { Invest }}\end{array}$ & $\begin{array}{l}\text { productivit } \\
\beta_{\omega^{2}, \text { Invest }}\end{array}$ & $\beta_{c \omega, \text { Invest }}$ & $\mathrm{N}$ \\
\hline $\mathrm{C} 10$ & $\begin{array}{c}1.32^{* * *} \\
(.15)\end{array}$ & $\begin{array}{c}.009 * * * \\
(.00)\end{array}$ & $\begin{array}{c}.00 \\
(.00)\end{array}$ & $\begin{array}{c}.577^{* * *} \\
(.06)\end{array}$ & $\begin{array}{c}.033^{* * *} \\
(.01)\end{array}$ & $\begin{array}{l}-.001 \\
(.00)\end{array}$ & 9482 \\
\hline $\mathrm{C} 11$ & $\begin{array}{c}3.906^{* * *} \\
(.5)\end{array}$ & $\begin{array}{l}.013 \\
(.01)\end{array}$ & $\begin{array}{l}0^{* *} \\
(.00)\end{array}$ & $\begin{array}{r}-.255 \\
(.16)\end{array}$ & $\begin{array}{c}.101 * * * \\
(.01)\end{array}$ & $\begin{array}{l}-.004 \\
(.00)\end{array}$ & 1297 \\
\hline $\mathrm{C} 13$ & $\begin{array}{c}-2.443^{* * *} \\
(.79)\end{array}$ & $\begin{array}{c}-.03^{*} \\
(.02)\end{array}$ & $\begin{array}{c}.001 * * * \\
(.00)\end{array}$ & $\begin{array}{c}1.653^{* * * *} \\
(.19)\end{array}$ & $\begin{array}{c}-.042^{* * * *} \\
(.01)\end{array}$ & $\begin{array}{l}.002 \\
(.00)\end{array}$ & 1929 \\
\hline $\mathrm{C} 14$ & $\begin{array}{c}1.315^{* * *} \\
(.43)\end{array}$ & $\begin{array}{c}.033^{* * *} * \\
(.01)\end{array}$ & $\begin{array}{c}.00 \\
(.00)\end{array}$ & $\begin{array}{l}.6^{* * * *} \\
(.14)\end{array}$ & $\begin{array}{c}.031 * * * \\
(.01)\end{array}$ & $\begin{array}{c}-.004^{* *} \\
(.00)\end{array}$ & 1053 \\
\hline $\mathrm{C} 15$ & $\begin{array}{c}3.416^{* * *} \\
(.99)\end{array}$ & $\begin{array}{l}.042^{*} \\
(.02)\end{array}$ & $\begin{array}{l}.001 * * \\
(.00)\end{array}$ & $\begin{array}{l}(.14) \\
-.04 \\
(.31)\end{array}$ & $\begin{array}{c}.079^{* * *} \\
(.02)\end{array}$ & $\begin{array}{c}-.008^{* *} \\
(.00)\end{array}$ & 469 \\
\hline $\mathrm{C} 16$ & $\begin{array}{c}-2.228^{* *} \\
(.93)\end{array}$ & $\begin{array}{l}.001 \\
(.01)\end{array}$ & $\begin{array}{l}0 * * \\
(.00)\end{array}$ & $\begin{array}{c}1.736^{* * *} \\
(.27)\end{array}$ & $\begin{array}{c}-.059^{* * * *} \\
(.02)\end{array}$ & $\begin{array}{l}.00 \\
(.00)\end{array}$ & 1994 \\
\hline $\mathrm{C} 17$ & $\begin{array}{l}.583 \\
(.46)\end{array}$ & $\begin{array}{l}.009 \\
(.01)\end{array}$ & $\begin{array}{c}.001 * * * \\
(.00)\end{array}$ & $\begin{array}{c}.883^{* * * *} \\
(.14)\end{array}$ & $\begin{array}{l}.004 \\
(.01)\end{array}$ & $\begin{array}{r}-.002 \\
(.00)\end{array}$ & 2257 \\
\hline $\mathrm{C} 18$ & $\begin{array}{l}-1.166 \\
(1.17)\end{array}$ & $\begin{array}{l}.002 \\
(.01)\end{array}$ & $\begin{array}{c}.002^{* * *} \\
(.00)\end{array}$ & $\begin{array}{c}1.362^{* * * *} \\
(.28)\end{array}$ & $\begin{array}{l}-.027 \\
(.02)\end{array}$ & $\begin{array}{l}-.002 \\
(.00)\end{array}$ & 1635 \\
\hline $\mathrm{C} 20$ & $\begin{array}{l}.403 \\
(.36)\end{array}$ & $\begin{array}{l}-.003 \\
(.01)\end{array}$ & $\begin{array}{l}0 * * * \\
(.00)\end{array}$ & $\begin{array}{c}.961^{* * * *} \\
(.1)\end{array}$ & $\begin{array}{l}-.002 \\
(.01)\end{array}$ & $\begin{array}{l}.00 \\
(.00)\end{array}$ & 4222 \\
\hline $\mathrm{C} 21$ & $\begin{array}{c}2.606^{* * *} \\
(.6)\end{array}$ & $\begin{array}{l}-.01) \\
-.012 \\
(.03)\end{array}$ & $\begin{array}{c}0^{*} \\
(.00)\end{array}$ & $\begin{array}{l}.253 \\
(.2)\end{array}$ & $\begin{array}{c}.051 * * * \\
(.02)\end{array}$ & $\begin{array}{l}.003 \\
.00)\end{array}$ & 913 \\
\hline $\mathrm{C} 22$ & $\begin{array}{c}1.397 * * * \\
(.47)\end{array}$ & $\begin{array}{c}.016^{* *} \\
(.01)\end{array}$ & $\begin{array}{l}0 * * \\
(.00)\end{array}$ & $\begin{array}{c}.695^{* * * *} \\
(.13)\end{array}$ & $\begin{array}{l}.016^{*} \\
(.01)\end{array}$ & $\begin{array}{c}-.002 * * \\
(.00)\end{array}$ & 4573 \\
\hline $\mathrm{C} 23$ & $\begin{array}{c}1.635 * * * \\
(.51)\end{array}$ & $\begin{array}{l}.018^{*} \\
(.01)\end{array}$ & $\begin{array}{l}.00 \\
(.00)\end{array}$ & $\begin{array}{c}.566^{* * * *} \\
(.15)\end{array}$ & $\begin{array}{l}.029^{* *} \\
(.01)\end{array}$ & $\begin{array}{r}-.002 \\
(.00)\end{array}$ & 3763 \\
\hline $\mathrm{C} 24$ & $\begin{array}{c}2.428^{* * *} \\
(.78)\end{array}$ & $\begin{array}{r}-.008 \\
(.01)\end{array}$ & $\begin{array}{l}.00 \\
(.00)\end{array}$ & $\begin{array}{l}.447^{*} \\
(.23)\end{array}$ & $\begin{array}{l}.029^{*} \\
(.02)\end{array}$ & $\begin{array}{l}.001 \\
(.00)\end{array}$ & 3275 \\
\hline $\mathrm{C} 25$ & $\begin{array}{l}.08 \\
(.57)\end{array}$ & $\begin{array}{r}-.004 \\
(.01)\end{array}$ & $\begin{array}{c}0 * * \\
(.00)\end{array}$ & $\begin{array}{c}1.109^{* * *} * \\
(.15)\end{array}$ & $\begin{array}{r}-.015 \\
(.01)\end{array}$ & $\begin{array}{l}.00 \\
(.00)\end{array}$ & 10430 \\
\hline $\mathrm{C} 26$ & $\begin{array}{c}4.793^{* * *} \\
(1.45)\end{array}$ & $\begin{array}{c}.111^{* * * *} \\
(.03)\end{array}$ & $\begin{array}{c}.001 * * * \\
(.00)\end{array}$ & $\begin{array}{l}.068 \\
(.29)\end{array}$ & $\begin{array}{c}.046 * * * \\
(.01)\end{array}$ & $\begin{array}{c}-.012^{* * * *} \\
(.00)\end{array}$ & 3664 \\
\hline $\mathrm{C} 27$ & $\begin{array}{l}.032 \\
(.47)\end{array}$ & $\begin{array}{c}.04^{* * * *} \\
(.01)\end{array}$ & $\begin{array}{l}.00 \\
(.00)\end{array}$ & $\begin{array}{c}1.03^{* * * *} \\
(.12)\end{array}$ & $\begin{array}{c}-.004 \\
(.01)\end{array}$ & $\begin{array}{c}-.005^{* * *} \\
(.00)\end{array}$ & 5063 \\
\hline $\mathrm{C} 28$ & $\begin{array}{c}1.541 * * * \\
(.57)\end{array}$ & $\begin{array}{c}-.021^{* *} \\
(.01)\end{array}$ & $\begin{array}{l}.00 \\
(.00)\end{array}$ & $\begin{array}{c}.779^{* * * *} \\
(.14)\end{array}$ & $\begin{array}{l}.004 \\
(.01)\end{array}$ & $\begin{array}{c}.003^{* *} \\
(.00)\end{array}$ & 11500 \\
\hline $\mathrm{C} 29$ & $\begin{array}{c}1.647^{* * *} \\
(.34)\end{array}$ & $\begin{array}{c}.028^{* * * *} \\
(.01)\end{array}$ & $\begin{array}{l}0 * * * \\
(.00)\end{array}$ & $\begin{array}{c}.518^{* * * *} \\
(.12)\end{array}$ & $\begin{array}{c}.035 * * * \\
(.01)\end{array}$ & $\begin{array}{c}-.005^{* * *} \\
(.00)\end{array}$ & 2974 \\
\hline C30 & $\begin{array}{c}.584 \\
(1.29)\end{array}$ & $\begin{array}{l}-.014 \\
(.02)\end{array}$ & $\begin{array}{l}.00 \\
(.00)\end{array}$ & $\begin{array}{l}.922^{* *} \\
(.36)\end{array}$ & $\begin{array}{r}-.001 \\
(.03)\end{array}$ & $\begin{array}{l}.002 \\
(.00)\end{array}$ & 1000 \\
\hline C31 & $\begin{array}{l}1.506 \\
(1.92)\end{array}$ & $\begin{array}{c}-.204^{* * *} \\
(.05)\end{array}$ & $\begin{array}{l}.00 \\
(.00)\end{array}$ & $\begin{array}{c}1.053^{*} \\
(.54)\end{array}$ & $\begin{array}{l}-.034 \\
(.04)\end{array}$ & $\begin{array}{c}.027^{* * *} \\
(.01)\end{array}$ & 1875 \\
\hline $\mathrm{C} 32$ & $\begin{array}{c}2.194 * * * \\
(.64)\end{array}$ & $\begin{array}{l}.003 \\
(.01)\end{array}$ & $\begin{array}{c}.001^{* * *} * \\
(.00)\end{array}$ & $\begin{array}{c}.522^{* * *} \\
(.17)\end{array}$ & $\begin{array}{c}.026^{* *} \\
(.01)\end{array}$ & $\begin{array}{r}-.001 \\
(.00)\end{array}$ & 3042 \\
\hline C33 & $\begin{array}{l}.149 \\
(.93)\end{array}$ & $\begin{array}{c}.023^{* *} \\
(.01)\end{array}$ & $\begin{array}{c}.001^{* * * *} \\
(.00)\end{array}$ & $\begin{array}{c}1.033^{* * * *} \\
(.2)\end{array}$ & $\begin{array}{r}-.005 \\
(.01)\end{array}$ & $\begin{array}{c}-.003^{* *} \\
(.00)\end{array}$ & 3171 \\
\hline
\end{tabular}

$* * * \mathrm{p}<0.01, * * \mathrm{p}<0.05,{ }^{*} \mathrm{p}<0.1$. Standard errors in parentheses. 
Table C.6: Results of robustness tests, by 2-digit industry, KBC Investment

\begin{tabular}{|c|c|c|c|c|c|c|c|}
\hline Industry & Constant & $\begin{array}{c}\text { Point estir } \\
\beta_{c, \text { Invest }}\end{array}$ & $\begin{array}{l}\text { tes of the la } \\
\beta_{c^{2}, \text { Invest }}\end{array}$ & $\begin{array}{l}\text { of motion } \\
\beta_{\omega, \text { Invest }}\end{array}$ & $\begin{array}{l}\text { productivity } \\
\beta_{\omega^{2}, \text { Invest }}\end{array}$ & $\beta_{c \omega, \text { Invest }}$ & $\mathrm{N}$ \\
\hline H49 & $\begin{array}{c}.697^{* * *} \\
(.03)\end{array}$ & $\begin{array}{c}-.02^{* * *} \\
(.00)\end{array}$ & $\begin{array}{l}.00 \\
(.00)\end{array}$ & $\begin{array}{c}.834^{* * * *} \\
(.01)\end{array}$ & $\begin{array}{l}-.001 \\
(.00)\end{array}$ & $\begin{array}{c}.006^{* * *} \\
(.00)\end{array}$ & 75957 \\
\hline H50 & $\begin{array}{c}-2.575^{* * * *} \\
(.29)\end{array}$ & $\begin{array}{r}-.006 \\
(.02)\end{array}$ & $\begin{array}{l}.00 \\
(.00)\end{array}$ & $\begin{array}{c}1.738^{* * *} \\
(.07)\end{array}$ & $\begin{array}{c}-.051^{* * *} * \\
(.00)\end{array}$ & $\begin{array}{l}.002 \\
(.00)\end{array}$ & 7454 \\
\hline H51 & $\begin{array}{c}-2.935^{* * *} \\
(.37)\end{array}$ & $\begin{array}{l}.007 \\
(.02)\end{array}$ & $\begin{array}{l}.00 \\
(.00)\end{array}$ & $\begin{array}{c}1.98^{* * * *} \\
(.09)\end{array}$ & $\begin{array}{c}-.078^{* * *} * \\
(.01)\end{array}$ & $\begin{array}{l}.001 \\
(.00)\end{array}$ & 1482 \\
\hline $\mathrm{H} 52$ & $\begin{array}{l}.338^{*} \\
(.18)\end{array}$ & $\begin{array}{c}-.01 * * \\
(.00)\end{array}$ & $\begin{array}{l}.00 \\
(.00)\end{array}$ & $\begin{array}{l}1.073^{* * * *} \\
(.05)\end{array}$ & $\begin{array}{c}-.016^{* * * *} \\
(.00)\end{array}$ & $\begin{array}{c}.002^{* * *} \\
(.00)\end{array}$ & 32179 \\
\hline H53 & $\begin{array}{c}.866^{* * * *} \\
(.17)\end{array}$ & $\begin{array}{c}-.026^{* * *} \\
(.01)\end{array}$ & $\begin{array}{l}.00 \\
(.00)\end{array}$ & $\begin{array}{c}.843^{* * *} * \\
(.05)\end{array}$ & $\begin{array}{l}.004 \\
(.00)\end{array}$ & $\begin{array}{c}.004^{* * *} * \\
(.00)\end{array}$ & 7820 \\
\hline J58 & $\begin{array}{c}.338 * * * \\
(.06)\end{array}$ & $\begin{array}{c}-.026^{* * * *} \\
(.00)\end{array}$ & $\begin{array}{c}-.001^{* * * *} \\
(.00)\end{array}$ & $\begin{array}{l}1.011^{* * * *} \\
(.02)\end{array}$ & $\begin{array}{c}-.017^{* * * *} \\
(.00)\end{array}$ & $\begin{array}{c}.007^{* * *} \\
(.00)\end{array}$ & 10931 \\
\hline J59 & $\begin{array}{c}.227^{* * *} * \\
(.07)\end{array}$ & $\begin{array}{l}.002 \\
(.00)\end{array}$ & $\begin{array}{c}-.002^{* * *} * \\
(.00)\end{array}$ & $\begin{array}{c}1.019 * * * \\
(.03)\end{array}$ & $\begin{array}{c}-.016^{* * *} * \\
(.00)\end{array}$ & $\begin{array}{c}.007^{* * * *} \\
(.00)\end{array}$ & 5869 \\
\hline J60 & $\begin{array}{c}.73 * * * \\
(.24)\end{array}$ & $\begin{array}{l}.016 \\
(.02)\end{array}$ & $\begin{array}{r}-.001 \\
(.00)\end{array}$ & $\begin{array}{c}.794^{* * *} * \\
(.07)\end{array}$ & $\begin{array}{l}.005 \\
(.01)\end{array}$ & $\begin{array}{l}.002 \\
(.00)\end{array}$ & 838 \\
\hline J61 & $\begin{array}{c}1.174^{* * *} \\
(.14)\end{array}$ & $\begin{array}{c}.036^{* * *} \\
(.01)\end{array}$ & $\begin{array}{l}.00 \\
(.00)\end{array}$ & $\begin{array}{c}.585^{* * * *} \\
(.06)\end{array}$ & $\begin{array}{c}.033^{* * *} \\
(.01)\end{array}$ & $\begin{array}{c}-.006^{* * *} * \\
(.00)\end{array}$ & 4212 \\
\hline J62 & $\begin{array}{c}2.92^{* * *} * \\
(.24)\end{array}$ & $\begin{array}{c}-.151^{* * *} * \\
(.01)\end{array}$ & $\begin{array}{c}.002^{* * *} \\
(.00)\end{array}$ & $\begin{array}{c}.728^{* * *} \\
(.06)\end{array}$ & $\begin{array}{c}-.007^{* *} \\
(.00)\end{array}$ & $\begin{array}{c}.015^{* * *} \\
(.00)\end{array}$ & 43097 \\
\hline J63 & $\begin{array}{c}-.863^{* * *} * \\
(.23)\end{array}$ & $\begin{array}{c}-.104^{* * *} \\
(.01)\end{array}$ & $\begin{array}{c}.002^{* * *} \\
(.00)\end{array}$ & $\begin{array}{c}1.482^{* * *} \\
(.05)\end{array}$ & $\begin{array}{c}-.045^{* * *} * \\
(.00)\end{array}$ & $\begin{array}{c}.01^{* * *} * \\
(.00)\end{array}$ & 10904 \\
\hline M69 & $\begin{array}{c}.806^{* * *} * \\
(.08)\end{array}$ & $\begin{array}{c}-.029 * * * \\
(.00)\end{array}$ & $\begin{array}{c}.001^{* * *} * \\
(.00)\end{array}$ & $\begin{array}{c}.909^{* * *} * \\
(.03)\end{array}$ & $\begin{array}{c}-.008^{* * *} * \\
(.00)\end{array}$ & $\begin{array}{c}.003^{* * *} * \\
(.00)\end{array}$ & 81249 \\
\hline M70 & $\begin{array}{c}1.618^{* * *} \\
(.17)\end{array}$ & $\begin{array}{c}-.012^{* *} \\
(.01)\end{array}$ & $\begin{array}{c}.002^{* * *} \\
(.00)\end{array}$ & $\begin{array}{c}.769 * * * \\
(.04)\end{array}$ & $\begin{array}{l}.004 \\
(.00)\end{array}$ & $\begin{array}{l}.00 \\
(.00)\end{array}$ & 32538 \\
\hline M71 & $\begin{array}{c}5.021^{* * *} \\
(.06)\end{array}$ & $\begin{array}{c}-.209 * * * \\
(.00)\end{array}$ & $\begin{array}{c}-.005^{* * *} * \\
(.00)\end{array}$ & $\begin{array}{c}.366^{* * * *} \\
(.01)\end{array}$ & $\begin{array}{c}-.007 * * * \\
(.00)\end{array}$ & $\begin{array}{c}.042^{* * * *} \\
(.00)\end{array}$ & 59139 \\
\hline M72 & $\begin{array}{c}5.27^{* * *} \\
(.59)\end{array}$ & $\begin{array}{c}.063^{* * *} * \\
(.02)\end{array}$ & $\begin{array}{c}.002^{* * *} \\
(.00)\end{array}$ & $\begin{array}{l}-.04 \\
(.13)\end{array}$ & $\begin{array}{c}.051^{* * *} \\
(.01)\end{array}$ & $\begin{array}{c}-.008^{* * *} * \\
(.00)\end{array}$ & 7208 \\
\hline M73 & $\begin{array}{c}-.664^{* * *} * \\
(.16)\end{array}$ & $\begin{array}{c}-.026 * * * \\
(.00)\end{array}$ & $\begin{array}{c}.002^{* * *} * \\
(.00)\end{array}$ & $\begin{array}{c}1.3^{* * *} \\
(.04)\end{array}$ & $\begin{array}{c}-.028^{* * *} * \\
(.00)\end{array}$ & $\begin{array}{c}.002^{* * * *} \\
(.00)\end{array}$ & 26002 \\
\hline M74 & $\begin{array}{c}1.699^{* * *} \\
(.17)\end{array}$ & $\begin{array}{c}-.02^{* * *} \\
(.01)\end{array}$ & $\begin{array}{c}.001^{* * *} \\
(.00)\end{array}$ & $\begin{array}{c}.617^{* * *} * \\
(.05)\end{array}$ & $\begin{array}{c}.017^{* * * *} \\
(.00)\end{array}$ & $\begin{array}{c}.002^{* * *} \\
(.00)\end{array}$ & 17242 \\
\hline M75 & $\begin{array}{c}-2.941^{* * *} \\
(.5)\end{array}$ & $\begin{array}{c}-.089 * * * \\
(.02)\end{array}$ & $\begin{array}{c}.002^{* * *} \\
(.00)\end{array}$ & $\begin{array}{c}2.076^{* * * *} \\
(.15)\end{array}$ & $\begin{array}{c}-.095 * * * \\
(.01)\end{array}$ & $\begin{array}{c}.01^{* * *} \\
(.00)\end{array}$ & 11214 \\
\hline N77 & $\begin{array}{c}.683^{* * *} \\
(.06)\end{array}$ & $\begin{array}{c}-.01 * * \\
(.00)\end{array}$ & $\begin{array}{c}.002^{* * *} \\
(.00)\end{array}$ & $\begin{array}{l}83^{* * *} \\
(.02)\end{array}$ & $\begin{array}{c}.006^{* * *} \\
(.00)\end{array}$ & $\begin{array}{l}-.001 \\
(.00)\end{array}$ & 18494 \\
\hline N78 & $\begin{array}{c}.88^{* *} \\
(.4)\end{array}$ & $\begin{array}{c}-.068^{* * *} \\
(.01)\end{array}$ & $\begin{array}{c}.002^{* * * *} \\
(.00)\end{array}$ & $\begin{array}{c}1.155^{* * *} * \\
(.08)\end{array}$ & $\begin{array}{c}-.025 * * * \\
(.00)\end{array}$ & $\begin{array}{c}.006^{* * *} * \\
(.00)\end{array}$ & 16880 \\
\hline N79 & $\begin{array}{c}-3.972^{* * *} \\
(.37)\end{array}$ & $\begin{array}{c}-.109 * * * \\
(.01)\end{array}$ & $\begin{array}{c}0^{* *} \\
(.00)\end{array}$ & $\begin{array}{l}2.185^{* * * *} \\
(.09)\end{array}$ & $\begin{array}{c}-.086^{* * *} * \\
(.01)\end{array}$ & $\begin{array}{c}.015^{* * * *} \\
(.00)\end{array}$ & 14240 \\
\hline N80 & $\begin{array}{c}1.835^{* * *} \\
(.3)\end{array}$ & $\begin{array}{c}-.066^{* * *} \\
(.01)\end{array}$ & $\begin{array}{c}.002^{* * *} \\
(.00)\end{array}$ & $\begin{array}{c}.72^{* * * *} \\
(.08)\end{array}$ & $\begin{array}{l}.004 \\
(.01)\end{array}$ & $\begin{array}{c}.007^{* * *} * \\
(.00)\end{array}$ & 6746 \\
\hline N81 & $\begin{array}{c}-.183 \\
(.16)\end{array}$ & $\begin{array}{c}-.046^{* * *} \\
(.00)\end{array}$ & $\begin{array}{c}.001^{* * *} \\
(.00)\end{array}$ & $\begin{array}{c}1.224^{* * *} * \\
(.04)\end{array}$ & $\begin{array}{c}-.027 * * * \\
(.00)\end{array}$ & $\begin{array}{c}.005^{* * *} * \\
(.00)\end{array}$ & 48348 \\
\hline N82 & $\begin{array}{c}1.57^{* * *} * \\
(.16)\end{array}$ & $\begin{array}{c}-.013^{* * *} * \\
(.00)\end{array}$ & $\begin{array}{l}.00 \\
(.00)\end{array}$ & $\begin{array}{c}.702^{* * * *} \\
(.04)\end{array}$ & $\begin{array}{c}.011^{* * *} \\
(.00)\end{array}$ & $\begin{array}{c}.003^{* * *} \\
(.00)\end{array}$ & 22407 \\
\hline S95 & $\begin{array}{c}5.561^{* * *} \\
(.51)\end{array}$ & $\begin{array}{c}.048^{* * *} \\
(.01)\end{array}$ & $\begin{array}{c}.001^{* * *} \\
(.00)\end{array}$ & $\begin{array}{c}-.309^{* *} \\
(.13)\end{array}$ & $\begin{array}{c}.075^{* * *} \\
(.01)\end{array}$ & $\begin{array}{c}-.007 * * * \\
(.00)\end{array}$ & 8586 \\
\hline
\end{tabular}

*** $\mathrm{p}<0.01, * * \mathrm{p}<0.05, * \mathrm{p}<0.1$. Standard errors in parentheses. 


\section{Appendix D. Additional graphs of productivity divergence}

Figure D.1: Evolution of Productivity in Service, 2009 to 2013

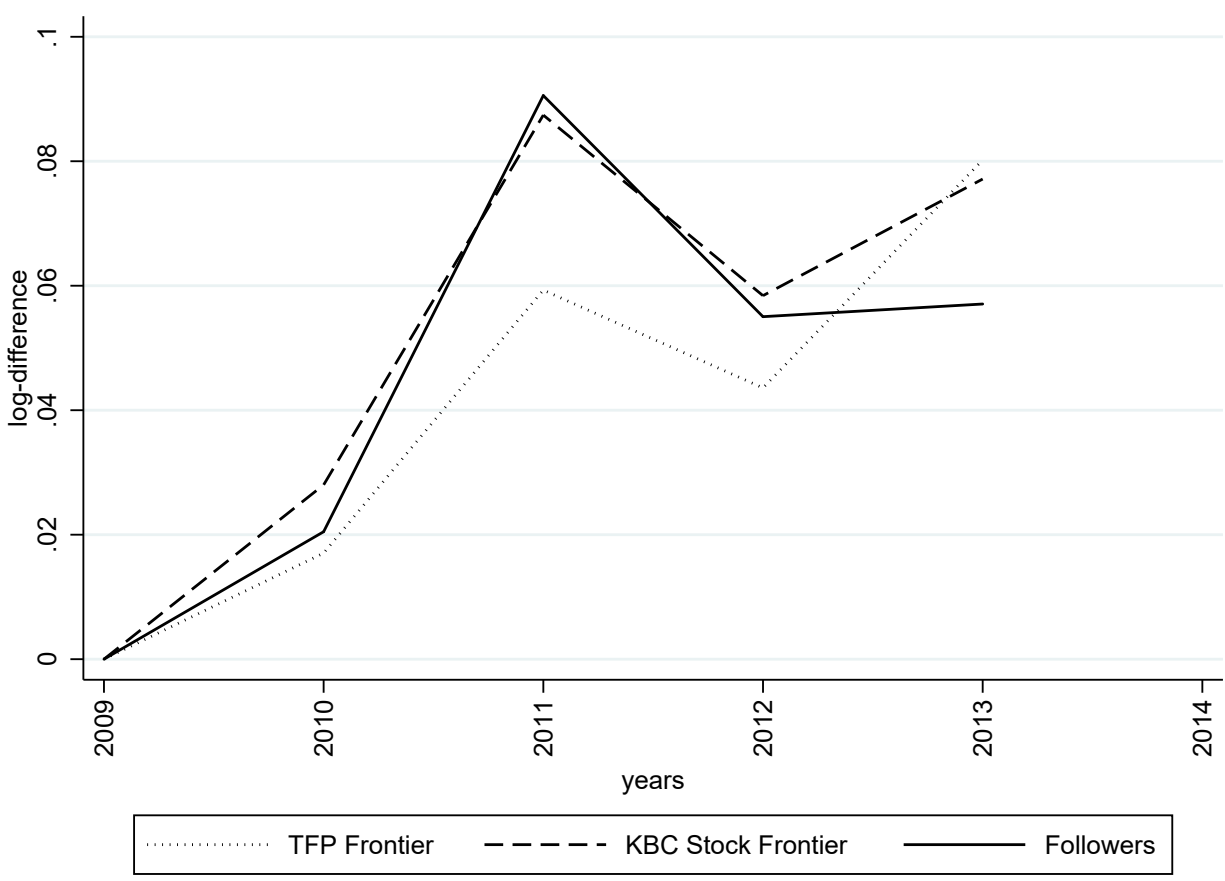


Figure D.2: Productivity gap of the KBC frontier at the 2-digit industry level

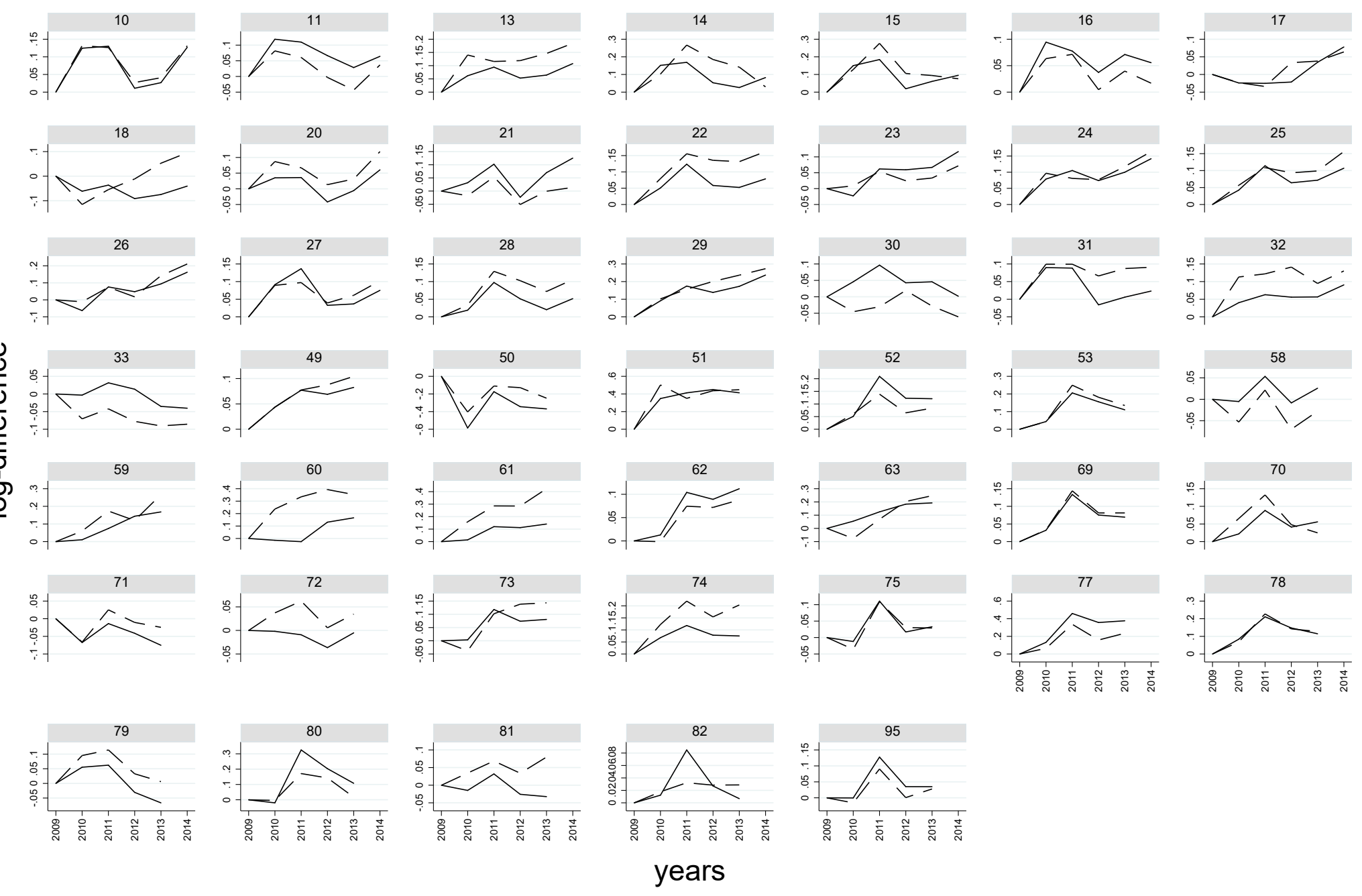

- - - - Frontier $\quad$ Followers


Figure D.3: Productivity gap of the TFP frontier at the 2-digit industry level
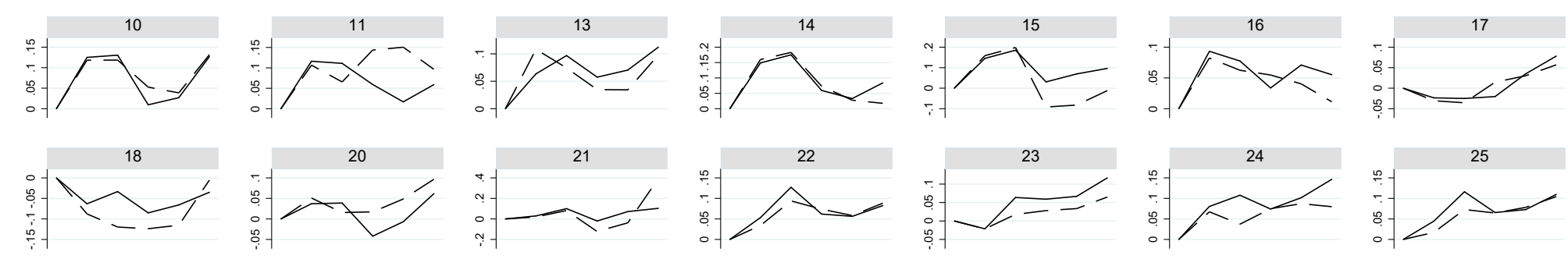

-

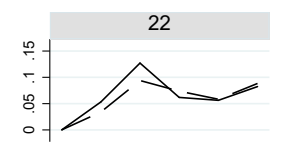

-
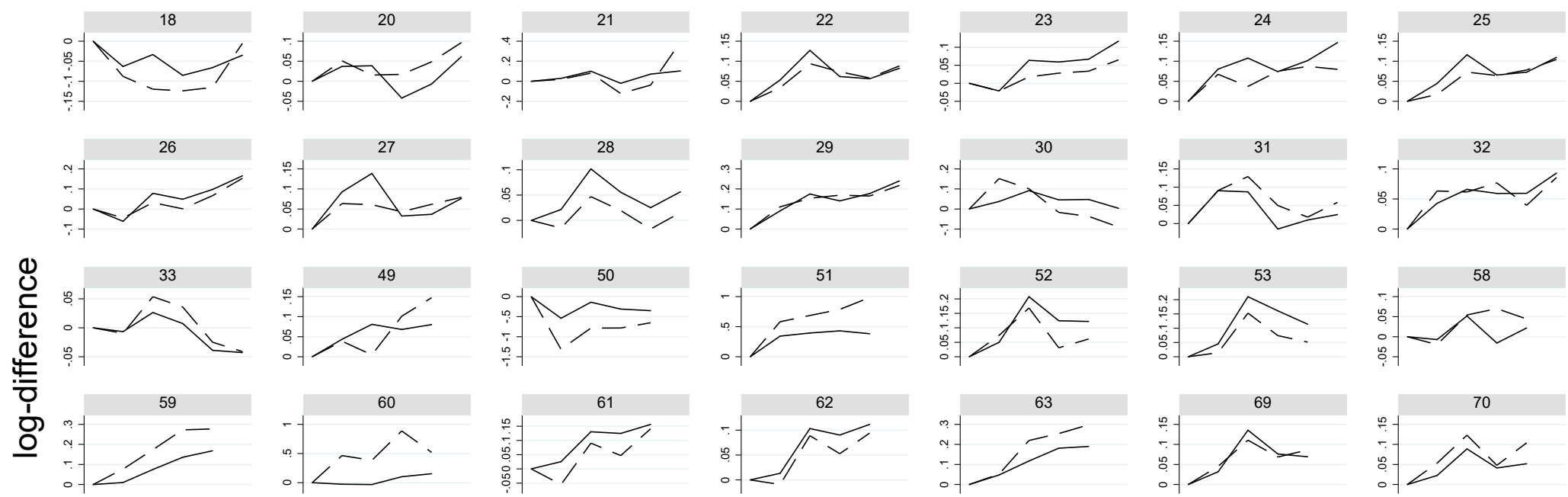

-

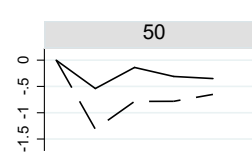

瓜
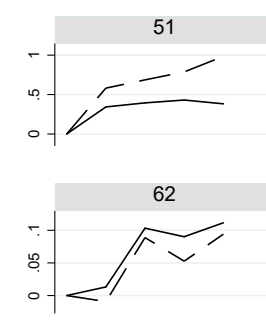

$\overbrace{-1}^{30}$
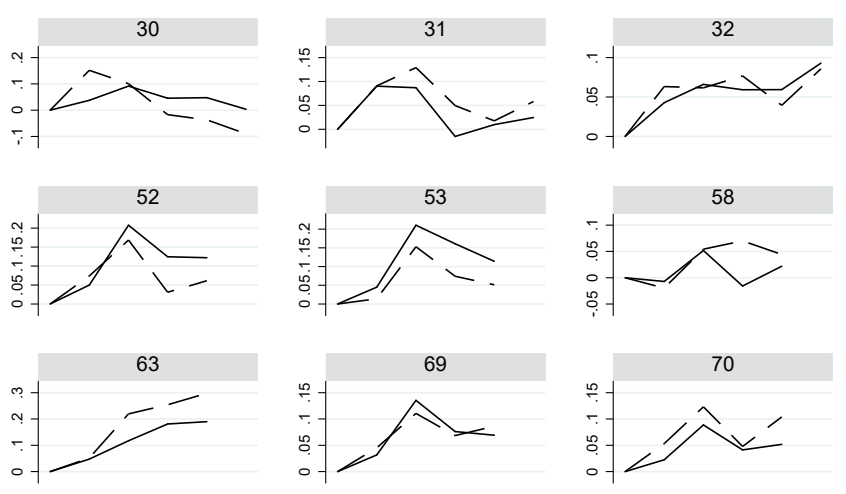

年
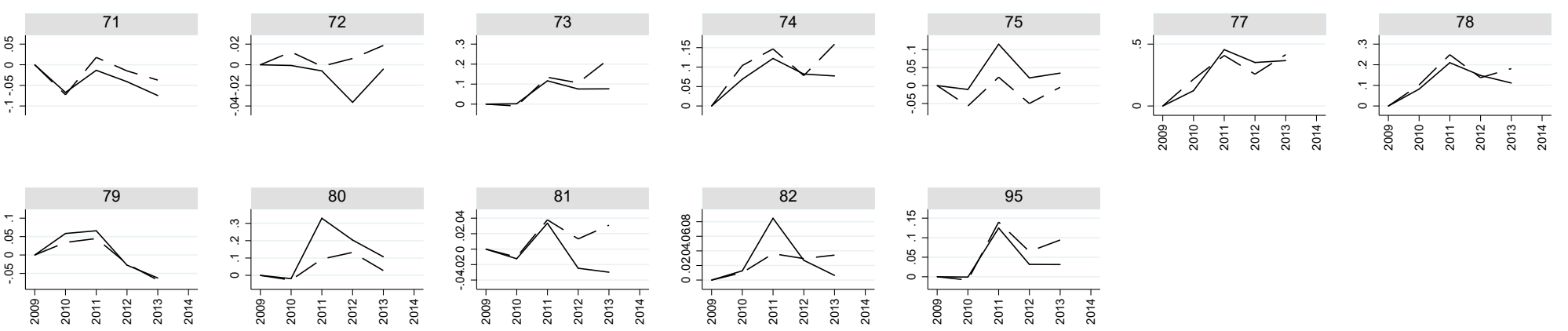
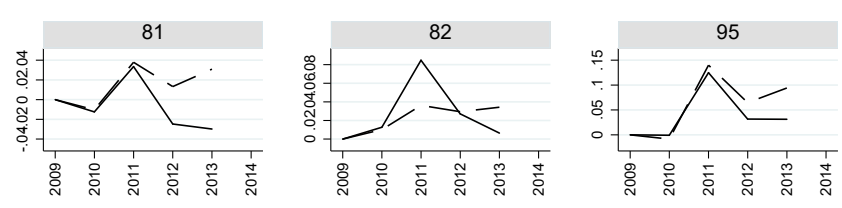

years

$$
\text { - - - - Frontier Followers }
$$


Appendix E. Details of the correlation between productivity gap and elasticity of KBC 
Figure E.1: Relationship between gap in productivity growth and measures of KBC elasticity in Manufacturing, according to KBC Stock frontier
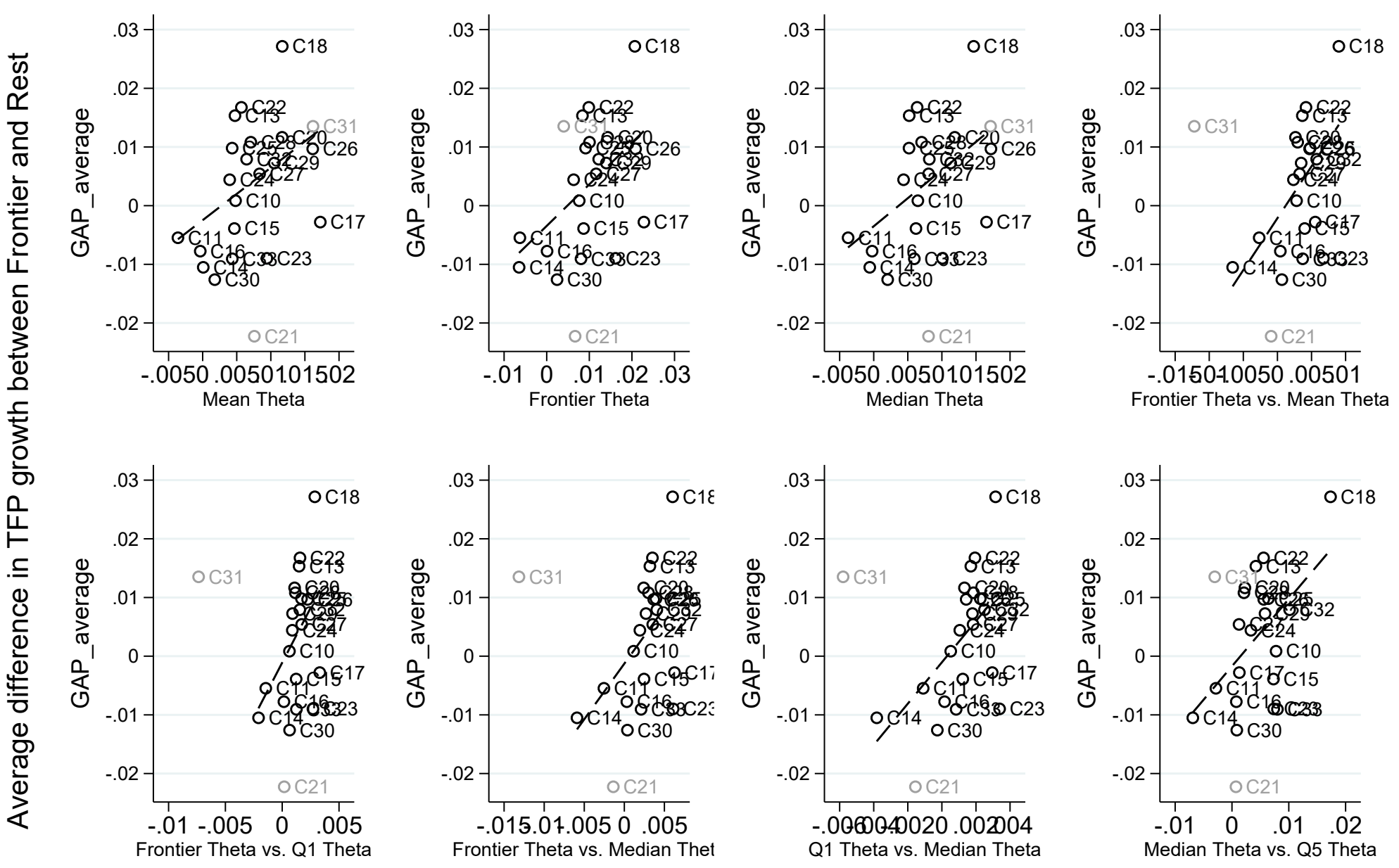

\section{O Manufacturing ----- Fit}


Figure E.2: Relationship between gap in productivity growth and measures of KBC elasticity in Services, according to KBC Stock frontier
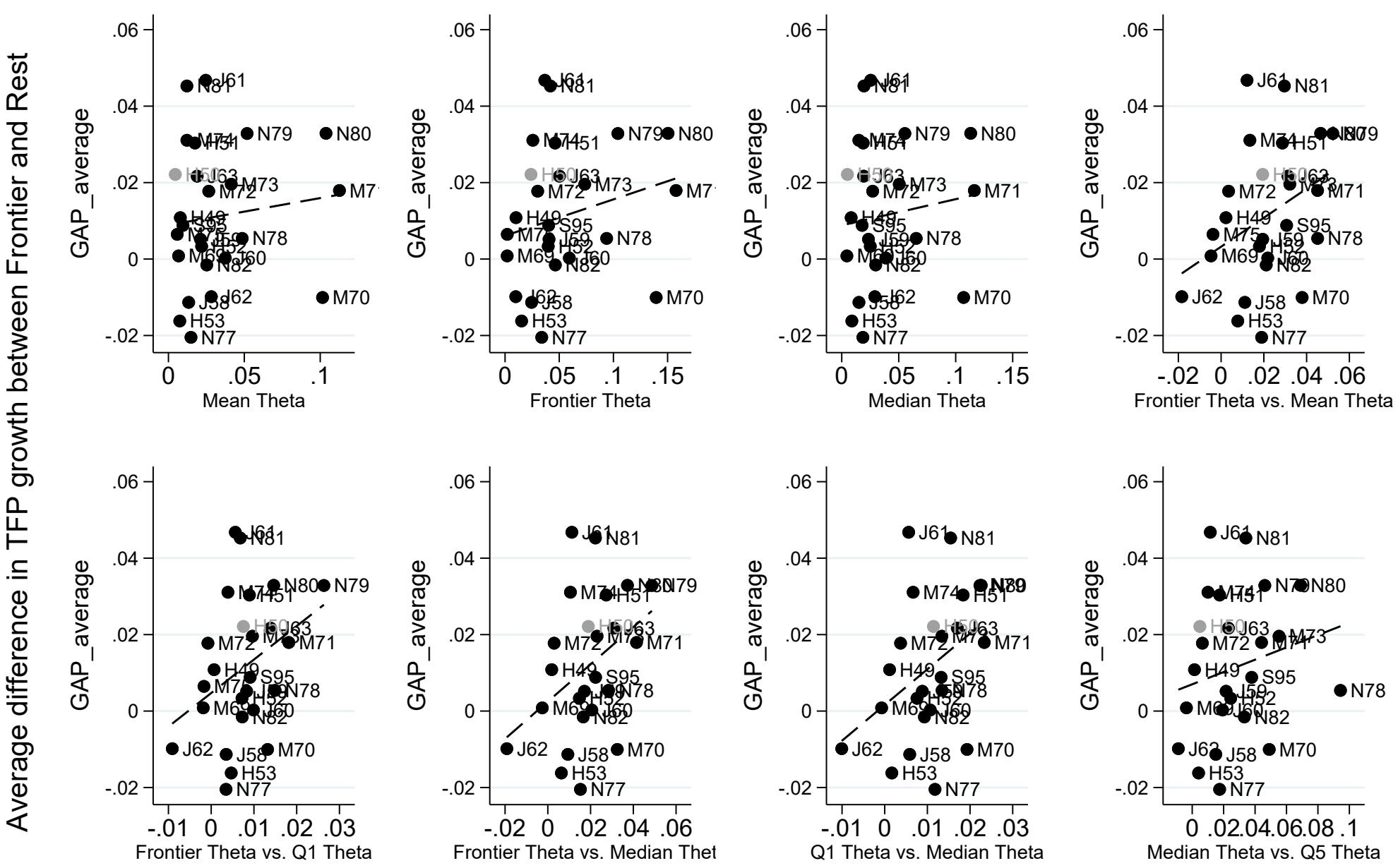

Services

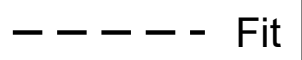

The unit of observations plotted are 2-digit industries. Industries C21, C31 and H50 are considered outliers, and thus plotted in light gray and not included in the estimation of the fitted line. 
Figure E.3: Relationship between gap in productivity growth and measures of KBC elasticity in Manufacturing, according to Productivity frontier
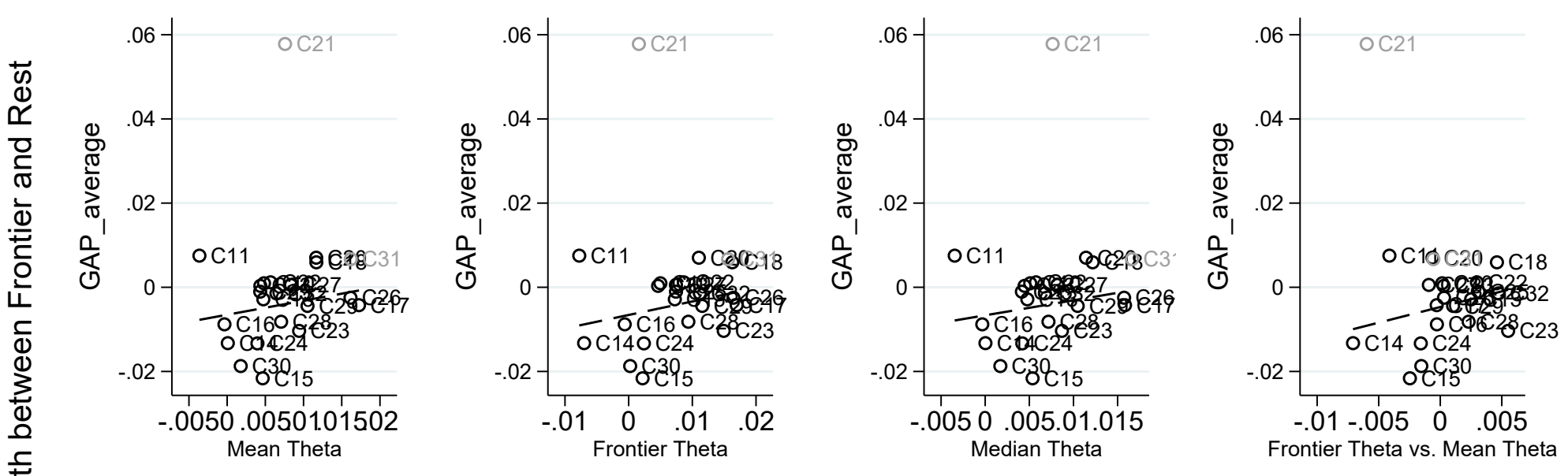

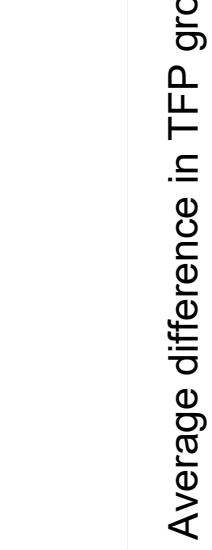
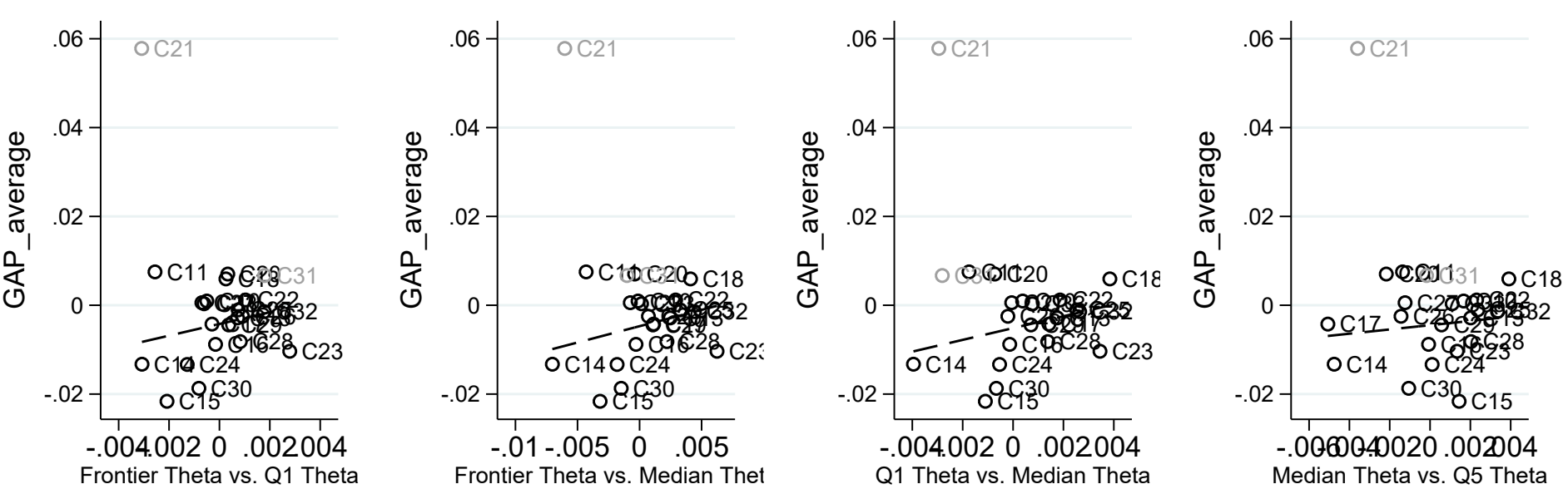

The unit of observations plotted are 2-digit industries. Industries C21, C31 and H50 are considered outliers, and thus plotted in light gray and not included in the estimation of the fitted line. 
Figure E.4: Relationship between gap in productivity growth and measures of KBC elasticity in Services, according to Productivity frontier
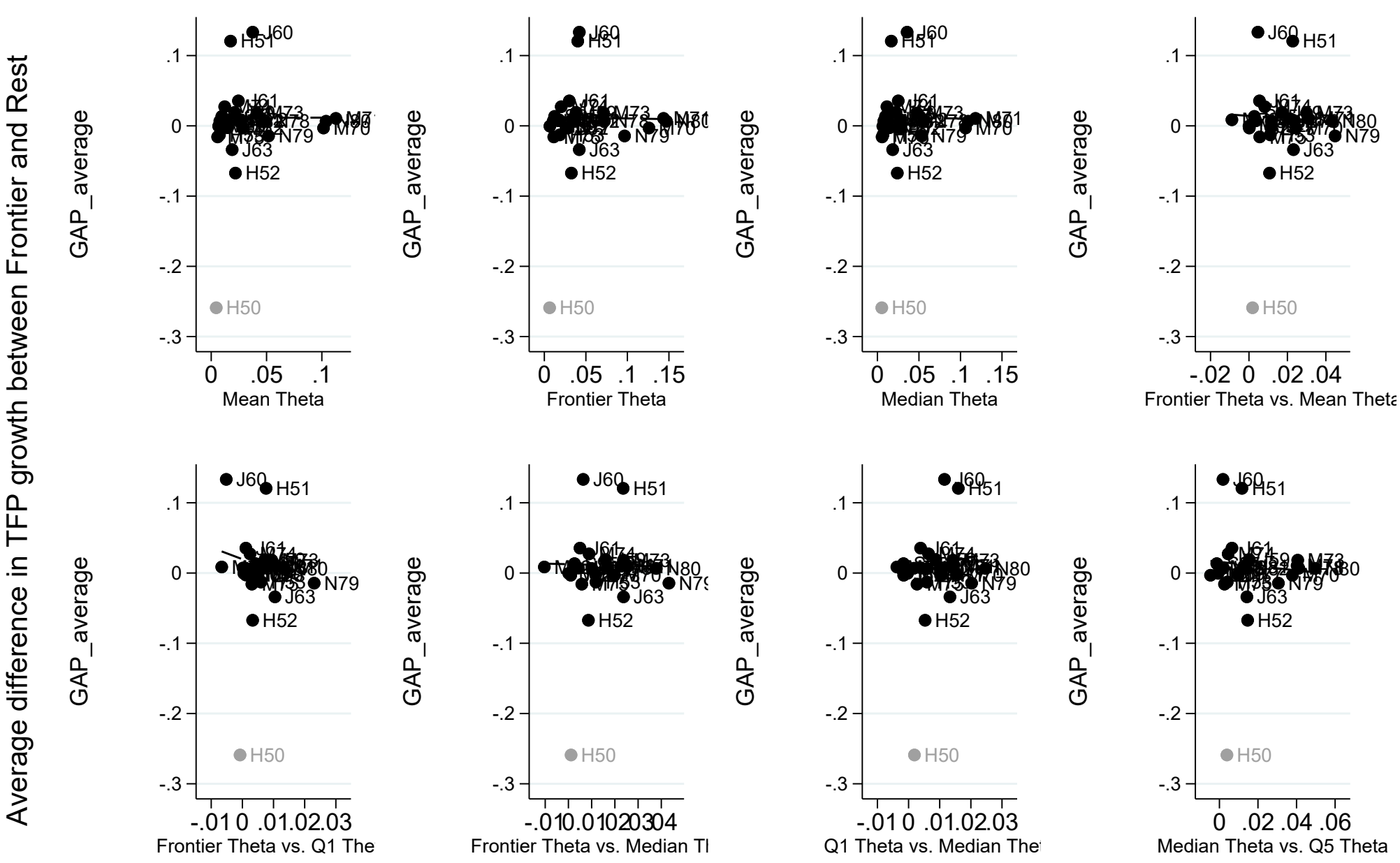

\section{Services - - - - Fit}

